\title{
Towards A Theory of Digital Network De/Centralization
}

\author{
Platform-Infrastructure Lessons Drawn from Blockchain \\ Enrico Rossi ${ }^{1} \&$ Carsten Sørensen ${ }^{2}$
}

\author{
Digital Innovation \\ Department of Management \\ The London School of Economics \& Political Science \\ London, UK \\ theory.digitalinfrastructures.org \\ www.digitalinfrastructures.org
}

\begin{abstract}
Global digital platforms are conquering the world and rely critically on digital infrastructures to function, yet little research has explored the fundamental interrelationship between the two. This working paper argues that understanding centralization and decentralization in digital networks as asymmetry and symmetry in mutual interdependencies between the constitutive elements of a digital network can help us understand the platform-infrastructure relationship more fundamentally (and vice versa). To this end, the paper proposes, as a starting point, the in-depth analytical and literature study of blockchain networks as a particularly revealing type of digital platform/infrastructure duality. The paper proposes an analytical model for characterizing de/centralization in digital networks and maps this onto blockchain networks. Based on this, the paper explores the de/centralization of blockchain, arguing that the extant blockchain literature largely has failed in providing a comprehensive understanding of de/centralization by not considering the complex second-order interdependencies between the different constitutive dimensions of a blockchain: the symbolic, technological and political dimension. Based on this, the paper provides an analysis of the meaning of de/centralization in blockchain networks by studying the interdependencies between its constitutive elements of coin, network technology, and social community.
\end{abstract}

Keywords: Digital Networks, Blockchain Analytical Framework, Definition, Decentralization, Digital Infrastructure, Digital Platform

This working paper is published as:

Rossi, Enrico and Sørensen, Carsten, Towards A Theory of Digital Network De/Centralization: Platform-Infrastructure Lessons Drawn from Blockchain (December 13, 2019). Available at SSRN: https://ssrn.com/abstract=3503609

It is also available at theory.digitalinfrastructures.org

The working paper can be used and cited as long as it is referenced as indicated above.

\footnotetext{
${ }^{1}$ https://www.lse.ac.uk/management/people/academic-staff/erossi https://www.linkedin.com/in/enrico-rossi-22a64a25/

${ }^{2}$ https://www.Ise.ac.uk/management/people/academic-staff/csorensen

http://carstensorensen.com

http://scholar.carstensorensen.com

https://www.linkedin.com/in/carsten/
} 


\section{Table of Contents}

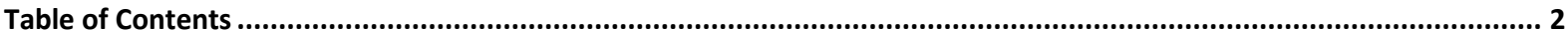

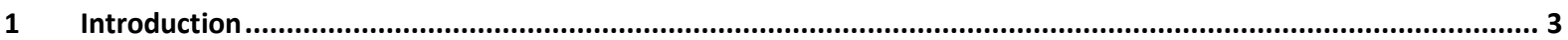

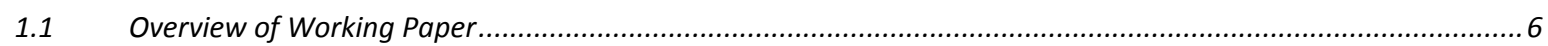

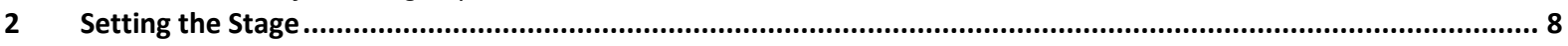

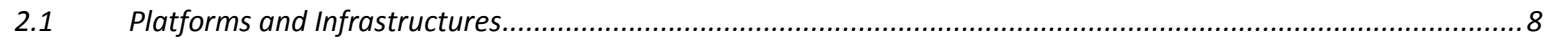

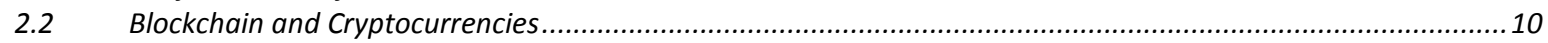

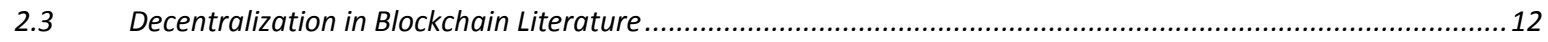

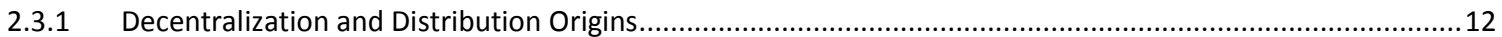

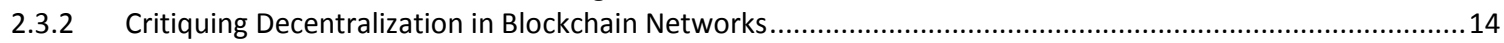

$2.4 \quad$ Problems with the Current Literature

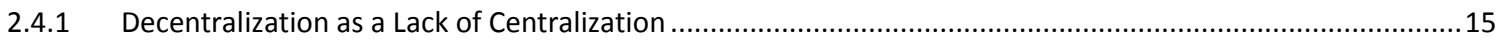

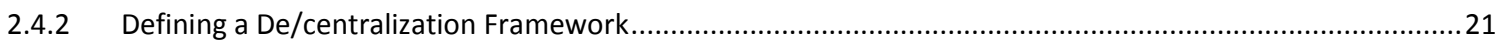

2.4.3 Defining de/centralization: a framework for the study of socio-technical system .......................................23

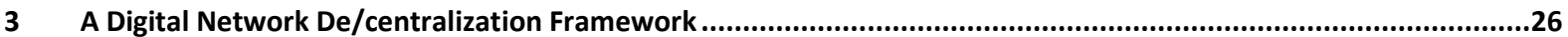

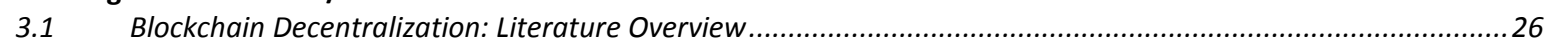

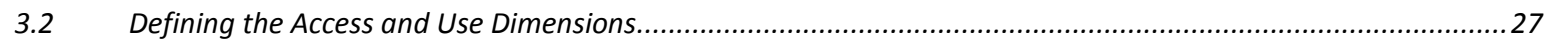

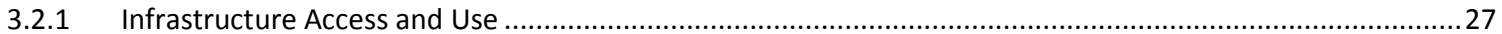

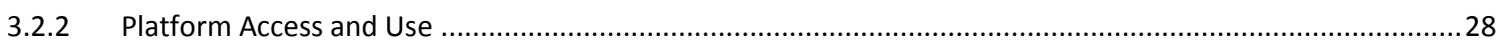

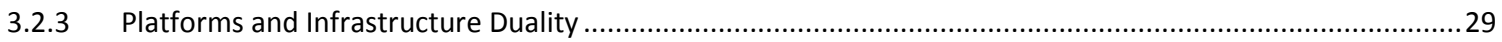

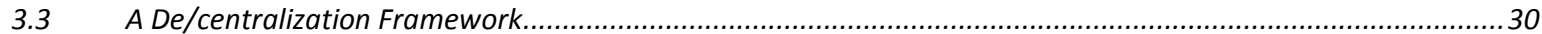

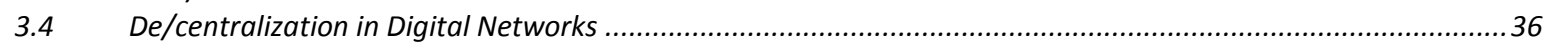

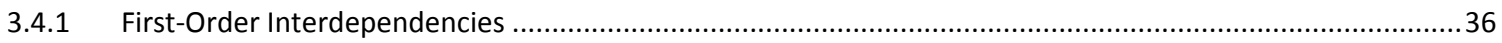

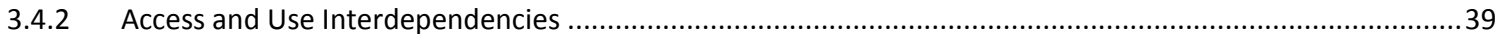

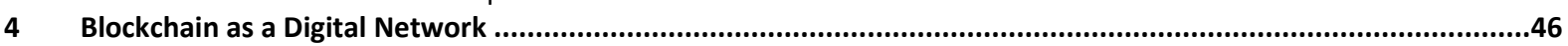

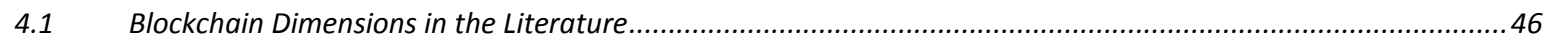

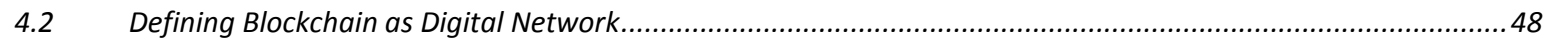

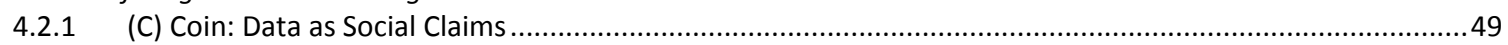

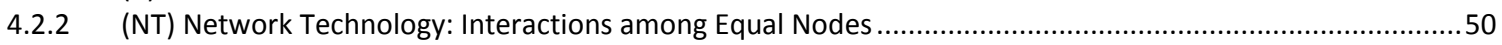

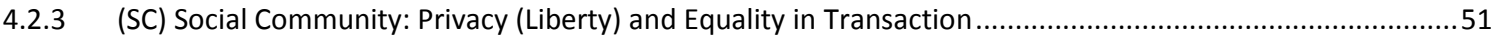

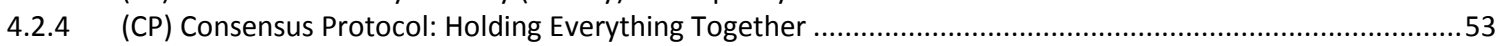

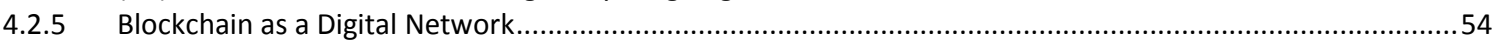

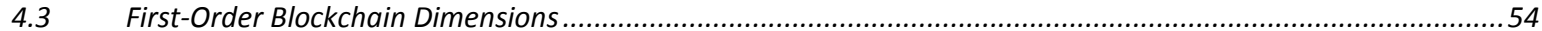

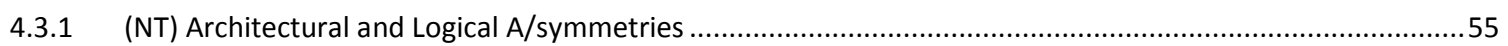

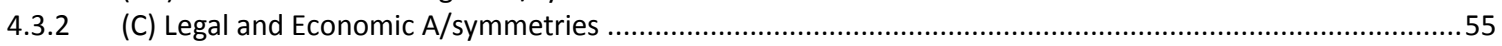

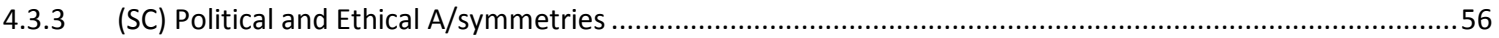

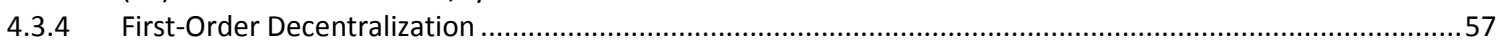

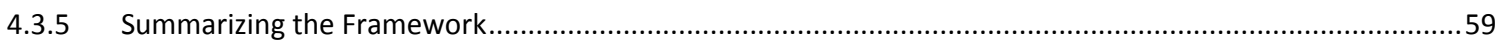

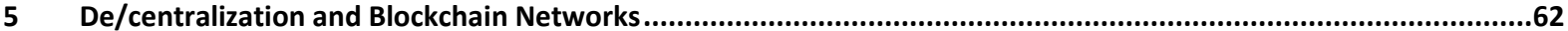

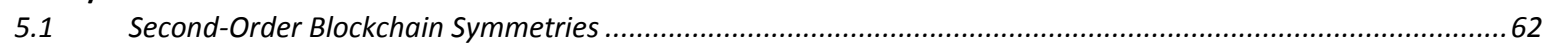

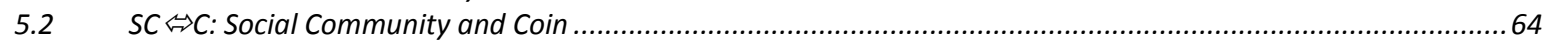

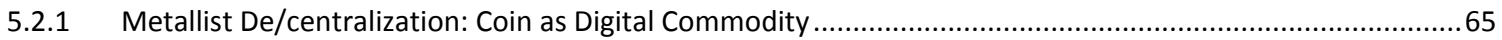

5.2.2 Informational De/centralization: Coin as Digital Fiat Money or Social Claim ..............................................69

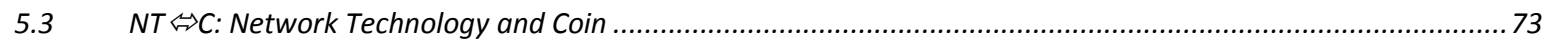

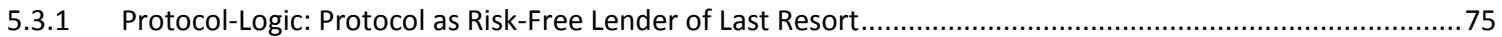

5.3.2 Permission-Logic: Protocol as a Neutral Validation Mechanism...............................................................78

5.3.3 Market-Power: Network as an Egalitarian Distribution of Capital..............................................................81

5.3.4 Summary of Techno-Economic Interpretations of De/centralization ...................................................... 84

5.3.5 Platform-Logic: Network as a Neutral and Open General-Purpose Technology............................................87

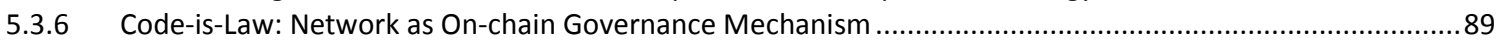

5.3.7 Principal-Agent: Network as Coordination Mechanism for On-Chain Division of Labour..............................91

5.3.8 Summary of Techno-Legal Interpretations of De/centralization ..............................................................95

5.4 NT $\Leftrightarrow S C$ : Network Technology and Social Community .............................................................................98

5.4.1 Social Community \& Information Sharing: Decentralization as Information Symmetries ...........................99

5.4.2 Social Community \& Information Sharing: Access and Control Symmetries .............................................101

5.4.3 Social Community \& Participation and Decision Rights: On-Chain Modification Symmetries .......................102

5.4.4 Social Community \& Political Power: Decentralization as Capacity to Influence.......................................103

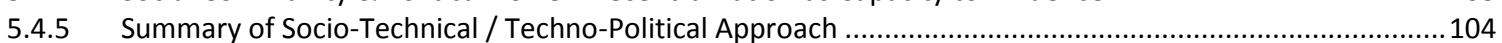

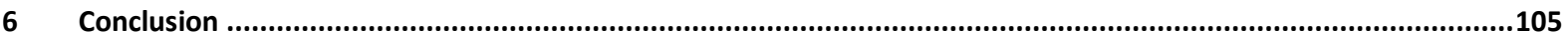

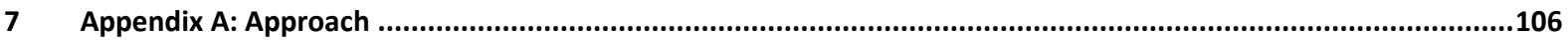

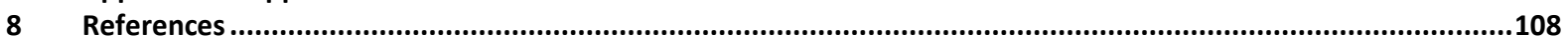




\section{Introduction}

Digital platforms and infrastructures play a critical role in the lives of individuals and in the functioning of societies. However, they have mostly been discussed in the literature within the implicit or explicit context of either an open Internet that supports the agnostic packaging, distribution, and re-assembly of data packages, or in terms of highly complex and organic evolved infrastructural arrangements characterized by innovation inertia. Core to the understanding of either of these discussions is the understanding of decentralization. In the case of the open Internet infrastructure, the relationship between centralization and decentralization can be characterized as through paradoxical forces of concurrent centralized and decentralized control (Tilson et al, 2010). The basic open Internet infrastructure has supported the highly decentralized exchange of data, and of unrestricted decentralized innovation of new services and protocols based on a common anchorpoint of the IP protocol. However, the secure exchange of economic or legal rights requires established institutional arrangements or digital platforms as mediators - hence the open Internet has been a petri-dish for an explosive growth of digital platforms of which a few has gained global dominance within their field of activity. Some of the most successful global digital platforms are blurring the platform/infrastructure boundaries by seeking to become infrastructural, for example through offering easy open authentication (de Reuver, 2018). Furthermore, much debate on these digital platforms are concerned with their central position in the harvesting of data, having excess market power, and distorting the political debate (Zuboff, 2019). In the contemporary academic debate on digital innovation, discussions of the interrelationships between digital platforms and infrastructures, the issue of de/centralization is, therefore, of core theoretical importance.

The aim of this working paper is to propose an analytical framework characterizing de/centralization in digital networks. ${ }^{3}$ This framework can subsequently be used for the in-depth analysis of de/centralization of digital platforms and -infrastructures in general. However, at this stage the framework will have most immediate impact for the theoretical framing of de/centralization within blockchain networks. ${ }^{4}$ The specific characteristics of blockchain networks render these particularly fertile in serving as the foundation for novel theorization on de/centralization since this has been one of the core issues under discussion within the broader blockchain community, and since blockchain networks cannot be considered as merely technological, cryptographic, organizational, personal, legal, or economic entities, but all of the above. The agnostic character of the IP-based open Internet "hour-glass" (Zittrain, 2008, 2019) implies that aspects and interdependencies that here easily can be abstracted, such as the content of data, in the blockchain context becomes an immediate concern.

\footnotetext{
${ }^{3}$ We will purposefully in this report avoid using the terms "platform" and "infrastructure" as theoretical constructs for our analysis as the aim of the framework is subsequently to serve as a means of unpacking the de/centralization of digital platforms and -infrastructures and, therefore, formulating the theory in terms of the object we wish to characterize is problematic.

${ }^{4}$ Please note that we assume readers have a basic understanding of blockchain and cryptocurrency mechanics to appreciate the argumentation forwarded, as the document does not provide such an introduction. A good source could be one of the books by DuPont (2019), Burniski \& Tatar (2017), Lacity (2019), or Tapscott \& Tapscott (2017). There are also brief introductions in the articles by Lacity and colleagues $(2018,2019)$, Beck et al (2018), or Zachariadis et al (2019). The Internet also has plenty of articles and videos to assist if necessary.
} 
We have not been able to identify any work attempting to establish a relationship between the inherent tensions generated by the interplay between the platform and the infrastructural dimension of any digital network and the tensions emerging from the centralizing forces in blockchain networks. Neither have we identified any work attempting to study the centralizing trends of blockchain networks in the light of the platform-infrastructure interplay even though an emerging literature in media studies (Helmond, 2015; Helmond et al., 2019; Nechusthai, 2018; Plantin et al., 2018; Plantin and Punathambekar, 2019), information system (Bygstad and Hanseth, 2018; Costantinides et al., 2018; Lorenz and Stefan, 2018; Kazan et al, 2018) and legal studies (Zittrain, 2018; Flew, 2019; Flew et al., 2019) is beginning to address the issue.

Blockchain is usually defined in the literature either as a platform (De Filippi and Wright, 2018: chapter 1; Glaser et al., 2019; Mattila and Seppälä, 2018; Narayanan et al., 2016: chapter 9; Perreira et al, 2019), or as an infrastructure (DeFilippi and McMullen, 2018; De Filippi and Wright, 2018: chapter 2; Finck, 2019: 11; Glaser, 2017), especially as a "financial infrastructure" (McWaters et al., 2016; Walch, 2015). Nakamoto (2008) never qualifies its distributed time-stamp system neither as platform nor as infrastructure, but Buterin (2014) defines Ethereum as a "decentralised application platform." The confusion might reflect the shift in attention from the original bitcoin use case, which tend to understand blockchain as an infrastructure as also originally envisioned (Nakamoto, 2008), to the rise of the new Ethereum-based use cases supported by general-purpose networks such as Hyperledger or Corda, which are often referred to as "platforms" (Lacity, 2018). ${ }^{5}$ But no work has so far attempted to define blockchain theoretically in function of the concepts of digital platform and infrastructure, and through this derive its necessary and sufficient characteristics in an analytical manner. This might be problematic when trying to discuss the meaning and nature of $\mathrm{de} /$ centralization in digital networks as our understanding of de/centralization might directly depend on the way in which the interplay between platform and infrastructure is understood. As a result, such a study of the fundamental characteristics of digital platforms and infrastructures, through the case of blockchain, will also in turn enable a theoretical discussion of the degree of de/centralization of a given blockchain in terms of the infrastructural and platform aspects of a digital network.

The working paper, therefore, extends a nascent stream of literature with the last few years witnessing an increasing interest in the governance of blockchain technology, and in the meaning of its "decentralized" nature. An emerging body of academic research seeking to make sense of this phenomenon is still very much in its infancy with relatively less work than warranted by the public debate. Although an increasing number of papers on blockchain governance is emerging especially, but not exclusively, in the legal literature, there is still a paucity of conceptual and theoretical works on blockchain grounded within management and Information Systems. Current exceptions are Beck et al's (2018) and Zachariadis et al's (2019) papers on blockchain governance, along with practitioner-oriented papers on corporate blockchain arrangements (Lacity, 2018; Beck et al 2019; Lacity et al, 2019).

The governing rationale of the report is that there is a lack of theoretical understanding of de/centralization in digital networks in general, and in the multi-disciplinary blockchain debate in

\footnotetext{
${ }^{5}$ The four limitations of Bitcoin recognised by Buterin (2014: 12-13) - lack of Touring completeness; value blindness; lack of state; blockchain blindness- can all be understood as more or less directly associated to the transition from infrastructures to platforms.
} 
particular. This paper seeks to address this issue by providing an analytical framework for digital networks, and critically to apply this in the context of blockchain networks. De/centralization is characterized in terms of interactional symmetries and asymmetries between the generic elements of Data, Network Technology, and Social Community, where these three elements belong to, respectively, three basic dimensions of a digital network: the symbolic dimension (the social meaning of value and rights), the technological dimension (the logic and architecture of the network), and the political dimension (moral principles norming and guiding the decisions of the community members). This is, in the context of blockchain arrangements translated into an investigation of the relationships between Coin (C), Network Technology (NT), Social Community (SC), and analysing how the addition of the element of the Consensus Protocol (CP) shapes their interaction.

We show how de/centralization can be described in terms of symmetrical and asymmetrical interdependencies between these elements across two different dimensions. Our first-order analysis of de/centralization considers the relationships within each of the three main constitutive elements (C, NT, and SC) within the context of a Consensus Protocol. For example, symmetry or asymmetry in the interdependencies between coins within a blockchain, between members of the social community, or between the nodes in the network. Our second-order analysis of de/centralization then considers the possible variation in the interdependencies between the constitutive elements in the analytical framework - between: (C $\Leftrightarrow \mathbf{S C}$ ) the coin and the social community; (C $\Leftrightarrow \mathbf{N T})$ the coin and the network technology; and (NT $\Leftrightarrow \mathbf{S C}$ ) the network technology and the social community.

The insights proposed in this report are gained from theoretically focusing the analytical study of de/centralization on the interdependencies between the constitutive elements in the framework, combined with the insight that de/centralization in digital networks must be considered as both first- and second-order interdependencies. This allows us, based on the study of the extant blockchain literature, to make theoretical claims on the diversity of aspects of de/centralization in blockchain networks. This is in contrast to the main thrust of the theoretical debate on blockchain de/centralization, which either takes decentralization as a given, or is based on one particular perspective, thus ignoring the way in which the interactions between different elements belonging to different dimensions can dynamically affect the nature of the overall system. This report disentangles these perspectives and provides a synthesis of a number of distinct de/centralization perspectives.

The main insight of the work regarding blockchain de/centralization is that while much blockchain research makes claims related to decentralization, there is a fundamental incongruity between the way in which blockchain (and digital network in general) is defined and the way in which de/centralization in understood. The analysis of blockchain in function of the core dimensions of digital platforms and digital infrastructures reveals that blockchain (as any digital network) is a multidimensional system defined by the dynamic interactions of multiple elements across different dimensions. As a result, in order to retain logical and analytical consistency we argue that all concepts qualifying blockchain should also be understood as multi-dimensional and dynamic (relational). While much blockchain research makes claims related to decentralization, then these claims are generally related to first-order and static interdependencies. However, decentralization at the level of the individual elements for one specific dimension of the digital network does not necessarily imply that the digital network can be characterized as de/centralized. The report 
discusses in significant depth and detail the diversity of de/centralization when considering secondorder interdependencies between network elements across the three different dimensions (symbolic, technological and political).

In the current version of the report, we exclusively relate the analytical model of digital network de/centralization to blockchain literature. It is the aim of the present work to provide the most comprehensive overview and detailed understanding of the nature and meaning of de/centralization in the case of blockchain. It is, however, the aim of subsequent work to draw more general conclusions on the applicability of the analytical framework to characterize de-centralization in digital networks in general. This could also allow us to make fundamental claims on a variety of digital platforms and infrastructures.

\subsection{Overview of Working Paper}

Practically, this working paper is in effect three interrelated, yet to some extent separate, arguments wrapped into one document. The paper also proposes a theoretical framework for analysing the first- and second-order interdependencies between the constitutive elements of a digital network. The paper then proposes a variant of this framework for the analysis of blockchain networks. Finally the paper applies this framework to the analysis of de/centralization in blockchain networks. This is, in more detail organized as follows:

Section 2 sets the stage by briefly outlining the main themes in the literature on digital platforms and -infrastructures. Then the section turns to an overview of the treatment of de/centralization within the discussions of blockchain and cryptocurrencies. The section then identifies the main issues with the current literature, namely, 1) the predominant underlying circular definition of blockchains being decentralized because they are based on blockchain technology; and 2) the monodimensional discussions of decentralization in an inherently multi-dimensional context. Section 2, furthermore, established the analytical categories, which will form the foundation for the further discussion, the: Technological (architectural and logical); economic, political, and legal interpretive approaches.

Section 3 develops a general framework characterizing de/centralization in digital networks, across the dimensions of use and access, consisting of the elements of: Data, Network Technology (Architectural and Logic), and Social Community. This section, furthermore, develops the notion of first-order and second-order systems of de/centralization.

Section 4 applies the analytical framework presented in Section 3 and translates it into the context of blockchain as a digital network. This translation presents the elements of the framework as: Coin (C), Network Technology (NT) - Architectural (A) and Logical (L), Social Community (SC), and the additional element of the Consensus Protocol (CP) - See Figure 1.1. The section argues that rather than considering de/centralization as either characteristics of each element, or indeed as merely first-order interdependencies, the notion should be understood in terms of second-order interdependencies between the elements. The section also proposes necessary and sufficient conditions for a network to be considered a blockchain, and discusses the types of systems emerging when one of the necessary conditions are not satisfied. Finally, the section argues that while the necessary and sufficient conditions may enable the definition of a blockchain, this does not 
necessarily imply that blockchains are decentralized as this aspect is not determined by the properties of first-order interdependencies, but of more complex second-order interdependencies.

Section $\mathbf{5}$ then in detail explores these second-order interdependencies by carefully in turn analysing the three interactions between the three main elements in the framework: (C $\Leftrightarrow \mathbf{S C}$ ) the coin social community interdependencies yielding the metallist and information perspectives on blockchain; (C $\Leftrightarrow \mathbf{N T}$ ) the coin - network technology interdependencies yielding the protocol-logic, the permission-logic, and the market power perspective within a techno-economic framing, and the platform-logic, the code-is-law, and the principal-agent perspective within a techno-legal framing; and finally, (NT $\Leftrightarrow \mathbf{S C}$ ) the network technology - social community interdependencies, discussing the critical and often neglected role of the social community to sustain and developments.

Section 6 provides a brief conclusion.

Section 7 - Appendix A: provides a brief characterization of the approach adopted for the literature study of more than 400 academic articles on blockchain.

Readers who are really mainly interested in a brief overview of an analytical framework for understanding blockchain and cryptocurrencies, can skip to Section 4. Each of Section 3, 4, and 5 contains summary sub-sections

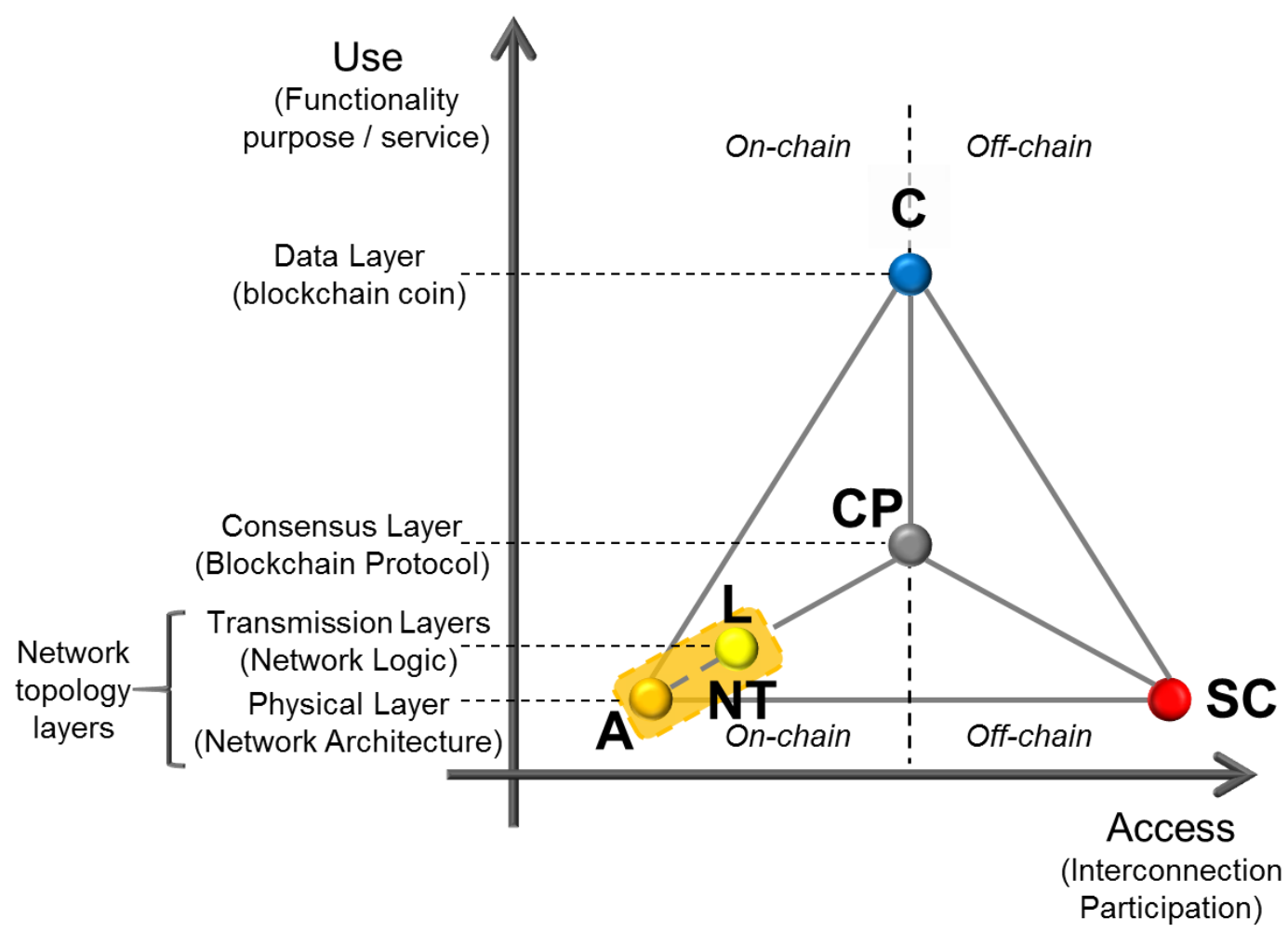

Figure 1.1: The analytical blockchain de/centralization framework. Constructed by projecting the general framework Data, Network Technology (Architectural, Logic), and Social Community onto the context of blockchain, replacing Data with Coin (C) with the addition of the consensus protocol (CP). 


\section{Setting the Stage}

This section briefly situates the issue of de/centralization within the context of: 1) digital platform and -infrastructure research; and 2) within the context of blockchain developments.

\subsection{Platforms and Infrastructures}

The recent years have witnessed the emergence of the platform concept as one of the core elements of the current socio-economic system. This increasing importance of platforms, or "platformization" has been characterised as "platform revolution" (Parker et al., 2016), "platform society" (van Dijck et al., 2018), or even "platform capitalism" (Srniceck, 2017). This is especially true with respect to the governance of digital ecosystems and, more specifically, of the internet.

Digital networks were originally analysed and understood as digital infrastructures, consistent with a long-standing tradition that conceived networks providing access to public service utilities as "infrastructures". This was true for the field of law (Frischmann, 2004 and 2012), economics (Valletti and Estache, 2001), communication policy (Musiani et al, 2016; Epstein et al, 2016), and information system (Ciborra et al., 2000; Edwards et al., 2007 and 2009; Tilson et al., 2010). This original focus on infrastructure was especially interested in the problems emerging from the interconnection of heterogeneous and situated actors, and from their open access and free use of the network, usually seen alternatively as a commons, essential facility, or common carrier. Since the mid-2000s, the concept of platform gained momentum in organization and management studies (Baldwin and Clark, 2000; Gawer and Cusumano, 2002; Gawer, 2009a; Parker et al., 2016), in legal studies (Farrell and Weiser, 2003; Page and Childers, 2012; Speta, 2002; Weiser, 2002), economics (Evans, 2011), sociology and media studies (Gillespie, 2010 and 2018; van Dijck, 2013) and information systems (Tiwana et al., 2010; de Reuver et al., 2018; Yoo et al., 2012). Consistent with the general trend, the concept of platform started to emerge as the dominant concept driving our understanding of digital networks. This "platformization of the web" (Helmond, 2015) - "data platforms" (Alaimo and Kallinikos, 2017), or "platformized internet" (Flew, 2019) - is founded on the conflation of infrastructural and platform elements of the digital networks, with a pronounced prominence of the latter (Andersson Schwarz, 2017; Flew, 2019; Helmond et al., 2019; Plantin et al., 2016; van Dijck et al., 2018).

The concept of platform has a relatively established and accepted meaning in organization and management studies (Gawer, 2014; Thomas et al, 2014), but this mainly derives from the fact that these disciplines usually do not need to contrast it to the infrastructural dimension of digital networks. When the study of infrastructures becomes core and essential, the situation becomes much more problematic. The academic literature has only very recently started to acknowledge that infrastructures and platforms are two distinct elements (or dimensions) of digital networks and that an analysis of the contribution of both dimensions is required (Constantinides et al., 2018; Plantin et al., 2016; Kazan et al, 2018; Plantin and Punathambekar, 2019). As a result, there is no clear understanding of what the platformization of an infrastructure precisely means in the context of digital networks, or what it entails. On the one hand, there is often no clarity on the way in which the two concepts shall be univocally characterised and distinguished from each other: infrastructures are very often intended and conceptualised as platforms (Benkler, 2006; Yoo et al., 2010; Zittrain, 2008), while platforms are often defined as infrastructures (Gillespie, 2018; van Dijck 
et al., 2018). This categorical confusion does not help in distinguishing the two concepts in the context of digital networks.

This transition from infrastructure to platforms has, however, not been interpreted in a univocal manner by the literature. Different authors have interpreted the interaction between the infrastructural and the platform dimensions of digital networks in different ways. Various authors have originally interpreted the platformized and modular nature of infrastructures as one of the fundamental characteristics of the digital networks, at the very heart of their generative, open and distributed nature, while the recent literature has started to interpret the platformization of digital infrastructures as a problematic characteristics of the internet, enabling centralized control and supervision over the transmitted content (de Reuver et al, 2018).

This divergence in opinions concerning the meaning and implications of the interplay between platforms and infrastructures certainly derives from the unclear nature of both concepts emerging from the paradoxical tensions inherent in both concepts of platforms (de Reuver et al., 2018; Tiwana et al., 2010; West, 2003; Yoo et al., 2012) and infrastructures (Edwards et al., 2009; Hanseth and Lyytinen 2010; Tilson et al., 2010). Both platforms and infrastructures can be simultaneously seen as determinants of centralization and decentralization depending on the way in which they are defined. As a result, these irreconcilable understandings of the two concepts, and of the implications of their linkage, also reveal a fundamental uncertainty with respect to the meaning and nature of both centralization and decentralization of a digital network. Depending on the point of view adopted, it can either be the infrastructural or the platform dimension of a network that ensures its decentralized nature.

Some authors, mainly rooted in the economic and legal approach, interpret infrastructures as natural monopolies and therefore as an inherent source of centralization. These actors regard the platformization emerging from the layered architecture of digital infrastructures as the necessary way through which decentralization can be introduced in digital networks. This is especially true for those approaches that focus on digital innovation and the generative potential of modular systems (Eaton et al., 2015; Gawer and Cusumano, 2008; Monteiro et al., 2013; Van Schewick, 2010; Yoo et al., 2012, Zittrain, 2006 and 2008), and on the "end-to-end" principle of the layered architecture that enables open innovation, collaborative production and diffused entrepreneurship (Autio et al., 2018; Benkler, 2006; Nambisan et al., 2018; Srinivasan and Venkatraman, 2018). Other authors perceive the platformization of the internet as a phenomenon fostering centralization of digital networks. According to this alternative interpretative approach platformization is a way to introduce centralized control points in the digital networks that can affect and influence the free and private use of the infrastructure and the free flow of data over it. In this case, platformization becomes a phenomenon breaching the end-to-end principle by shifting control away from the edges of the network, rather than a way to support it (Flew, 2019; Gilespie, 2018; Lemley and Lessig, 2001; Lessig, 2006; Wu, 2010; Zittrain, 2018).

The first stream of works - linking platformization to decentralization in infrastructures - mainly links the platform concept to the layered modular architecture of the internet (Greenstein, 2009; Hanseth and Lyytinen 2010; Van Schewick, 2010; Yoo, 2010), and conceives these as neutral and agnostic elements ensuring the decoupling between the structural and ontological characteristics of the underlying infrastructure and its use or functionalities (Kallinikos et al., 2013; Yoo et al., 2010). 
The second stream of works views platforms as intermediaries or control points that not only enable interconnection, but purposefully organize, shape and influence social interactions within an ecosystem (de Reuver et al, 2018; Jacobides et al, 2018). The introduction of intermediating platforms became necessary with the emergence of Web 2.0 where the sharing, exchange and transaction of user-generated content among dispersed peers of a network had to be organized, rationalized, aggregated and coordinated (Goldsmith and $\mathrm{Wu}, 2006$ ). This centralized intermediation introduces new issues as it necessarily requires some form of surveillance, inspection, filtering and censorship (more generally, control) of the data and content transmitted over the infrastructure (Deibert el al., 2010; Fuchs, 2011 \& 2013; Gillespie, 2010 and 2018; Nieborg and Poell, 2018; Srnicek, 2017; Trottier, 2016 and 2019; van Dijck, 2013; van Dijck et al., 2018; Zittrain and Palfrey, 2008; Zuboff, 2019). Both the economic literature on two-sided platforms (Rochet and Tirole, 2003,2006; Evans and Schmalensee, 2005) and the managerial literature on business ecosystems (Adner, 2017; Baldwin, 2012; Gawer, 2009a; Gawer and Henderson, 2007; Gulati et al., 2012; Jacobides et al 2018; Kapoor and Agarwal 2017; Thomas et al., 2014) mainly belong to this second interpretation of platform, conceived as a focal hub or intermediary that exercises some central organizing or coordinating function over the wider networked system.

To summarize, the current literature on platforms and infrastructures has not provided a univocal answer to the question of whether the platformized nature of digital infrastructures is a centrifugal force fostering decentralization, or rather a centripetal trend towards an increasing centralization of digital networks. The first and original interpretation of platformization as the essence of the layered, agnostic, and decentralized internet infrastructure was essential for the development of the first Web in the early 1990s. The second, more recent interpretation, views the platformization of the internet a centralizing force of an otherwise decentralized network. This interpretation mainly emerged in the early 2000s, with the transition towards the "networked sociality" of the Web 2.0 (van Dijck, 2013: chapter 1). A better understanding of the meanings of both concepts of platform and infrastructure will help in clarifying how to interpret the de/centralized nature of a digital network.

It is precisely in light of this second trend, and in response to its novel problems that blockchain technology was developed as the technology supporting the exchange of bitcoin. Blockchain can be mainly interpreted as a response to the centralizing forces emerging from the platformization of digital infrastructures in Web 2.0. Consistent, the founding fathers of blockchain adopted an interpretative approach consistent with a Web 2.0 view of platformization of a digital infrastructure.

\subsection{Blockchain and Cryptocurrencies}

The 2008 global financial crisis has a lot to answer for in terms of, for example, the possible links to the rise of populism in the West. However, it also provided an anchor-point bringing together communities with divergent interests into a global movement seeking to use digital technologies to bring about a new financial system. When an individual or group of people under the pseudonym Satoshi Nakamoto (2008), published a whitepaper outlining such a new system, the subsequent efforts of establishing the Bitcoin blockchain unified people motivated by the desire for pseudonymous peer-to-peer cash, with those desiring for a deflationary financial system (Swartz, 2018; Kavanagh et al., 2019). The subsequent decade has seen a rollercoaster ride spawning the Bitcoin open source protocol into many new cryptoassets, the establishment of Ethereum as an infrastructure for smart contracts, the 2017 explosive growth in crowd financing of blockchain-based 
startups through Initial Coin Offerings (ICOs), and fluctuating cryptoasset prices. The 2017 price hike of bitcoin and other cryptoassets was followed by a rapid decline. Subsequently, much discussion amongst practitioners has been concerned with the viability of so-called "public permissionless" blockchains, such as Bitcoin, as opposed to equally so-called "private permissioned" blockchains offering possibilities for closed consortia of firms to engage in streamlined collaboration. In June 2019, The Libra Association was announced. It is a consortium of just under 30 global firms coordinated by Facebook aiming at supporting Peer-to-Peer (p2p) transfer of funds through blockchain technology.

Blockchain has been a buzzword for the past few years with few subjects drawing such a broad scope and diverse opinions and emotions. Blockchain seems to be both all things to all people with some being devotees and others deeply critical. One of the key reasons for the diversity of opinions is a poor public debate littered with preconceptions and misunderstanding. ${ }^{6}$ Only in the last few years the literature has started to take blockchain seriously and to grasp its peculiar nature.

A brief look at all the main writings of the early supporters of bitcoin show clear concerns with the novel problems posed by intermediaries acting as centralized points of control prone to surveillance, censorship, filtering and manipulation: from cryptographer David Chaum's first framework for an untraceable electronic cash in the 1980s, ${ }^{7}$ over Hal Finney's Cypherpunks' writings in the $1990 s^{8}{ }^{8}$ to the inventor of the notion of smart contracts, Nick Szabo's writings in computer science, law and economics. ${ }^{9}$

The bitcoin blockchain was eventually developed in order to reintroduce decentralization in platformized networks subject to increasing centralization and to mitigate the potential associated risks. As stated by Satoshi Nakamoto in one of their letters to peers in the winter of 2008: "Governments are good at cutting off the heads of a centrally controlled networks like Napster, but pure peer-to-peer (p2p) networks like Gnutella and Tor seem to be holding their own" (Nakamoto, 2008). Here, centralization emerges following the introduction of a platform acting as a third-party intermediary in a peer-to-peer network. Platforms and infrastructures have univocal meanings; they do not manifest an ambiguous dual nature. As a result, the original interpretation of platformization,

\footnotetext{
${ }^{6}$ References goes from authors claiming cryptocurrencies are pure speculative bubbles (https://www.cnbc.com/2019/05/08/nourielroubini-and-michael-novogratz-battle-over-bitcoin-and-crypto.html; https://cointelegraph.com/news/nouriel-roubini-at-salt-conferencein-ny-crypto-is-the-mother-and-father-of-all-bubbles), to blockchain being completely useless (https://cointelegraph.com/news/nourielroubini-cryptocurrency-as-a-technology-has-absolutely-no-basis-for-success), or a Ponzi scheme.

7 "The use of credit cards today is an act of faith on the part of all concerned. Each party is vulnerable to fraud by the others, and the cardholder in particular has no protection against surveillance. Paper cash is considered to have a significant advantage over credit cards with respect to privacy, although the serial numbers on cash make it traceable in principle." (Chaum et al., 1988: 319; emphasis added).

8 "One concern I have is that the move to electronic payments will decrease personal privacy by making it easier to log and record transactions. Dossiers could be built up which would track the spending patterns of each of us ... I think that the convenience of credit and debit cards will overcome most people's privacy concerns and that we will find ourselves in a situation where great volumes of information exist about people's private lives. This is a place that I could see digital cash playing a role ... [as] still no central location is able to track everything I buy." (Finney, 1993; emphasis added).

9 "Commercial security is a matter of solving the practical problems of business relationships such as privacy, integrity, protecting property, or detecting breach of contract ... The invocation or assumption in a security protocol design of a "trusted third party" (TTP) or a "trusted computing base" (TCB) controlled by a third party constitutes the introduction of a security hole into that design. The security hole will then need to be plugged by other means." (Szabo, 2001: 1).
} 
intended as a layered modular architecture of an agnostic and generative network, is absent among early enthusiasts of the bitcoin's blockchain technology, and it will only be partially recovered after the introduction of Ethereum).

Yet, in the same way in which the evolution of digital networks has shown a confusing swing from centralization to decentralization due to the dual nature of platforms and infrastructures, the development of blockchain was also affected by the same fate. Starting with the 2013 hack of the Japanese cryptocurrency exchange Mt. Gox, ${ }^{10}$ it became clear that a technology that was specifically developed in order to counteract the centripetal trends of the platformized Web 2.0 concealed similar tendencies and the same incentives towards centralization. By the 2016 DAO (Distributed Autonomous Organization) ${ }^{11}$ hack it was apparent that the technology was in fact exhibiting the same type of tendencies towards centralization of the internet network (DuPont, 2019). This spurred a debate among practitioners and experts in the sector on the meaning of decentralization and on the way in which this concept should be intended in the in blockchain space (DuPont, 2018; Musiani et al., 2018). The launch of the Libra project lead by Facebook could only reinvigorate a debate on whether blockchain really addresses and counteracts the re-centralizing tendencies of platformized digital infrastructures, or if it will inexorably follow the same fate. ${ }^{12}$

The global innovation effort seems to be seeking two different goals through blockchain technology. The first is to establish a distributed "internet of value" (Web 3.0) based on a mixture of "public permissionless" and "private permissioned" blockchain technology, and the second is comprised of efforts to establish blockchains for business use (Lacity, 2018; DuPont, 2019). The "public permissionless" community frequently relate to Baran's (1964) architectural distinction between centralised, decentralised, and distributed systems in the emphasis on fully distributed $p 2 p$ transactions. The "private permissioned" debate emphasises business-to-business interaction within closed consortia.

The following will seek to establish a conceptual framework allowing for a more rigorous unfolding of these concepts characterizing de/centralization in digital networks in general, and blockchain networks in particular. As will be evident from our discussion, the issue of de/centralization is a good deal more multi-faceted than the mainstream academic and practitioner debate so far has acknowledged.

\subsection{Decentralization in Blockchain Literature}

This section reviews the blockchain literature in order to establish the foundational categories for the following discussion of de/centralization.

\subsubsection{Decentralization and Distribution Origins}

Blockchain, and the tokens transacted over it, are usually defined as a decentralized. The term "decentralization" does not appear in Satoshi Nakamoto' 2008 white paper (Nakamoto, 2008: 1), nor

\footnotetext{
${ }^{10}$ https://en.wikipedia.org/wiki/Mt._Gox

${ }^{11}$ https://en.wikipedia.org/wiki/The_DAO_(organization)

https://en.wikipedia.org/wiki/Privacy_and_blockchain\#DAO_Hack

${ }^{12}$ A good summary of the debate over the decentralized nature of Libra is provided by this response article of the FT "Facebook fights back against Libra criticism" (https://ftalphaville.ft.com/2019/07/05/1562323175000/Facebook-fights-back-against-Libra-criticism/)
} 
in any of the emails exchanged between Satoshi and friends between 2008 and $2009 .{ }^{13}$ Nevertheless, Nakamoto's "peer-to-peer distributed timestamp server" (Nakamoto, 2008: 1) was developed in order to overcome centralization in digital payment networks and to devise an alternative to "the standard common solution [...] to introduce a trusted central authority". Building on the same concerns with centralized control points (or intermediaries) Ethereum was originally defined as a "Decentralized Application Platform" (Buterin, 2014; Wood, 2014) and bitcoin was defined by Buterin (2014: 1) as a "decentralized currency" even though Nakamoto never used the term. The term "decentralized" will also appear in the name of the most famous enterprise set up on the Ethereum network: the DAO (Decentralized Autonomous Organization) (Jentzsch, 2016).

Since then, the terms decentralization and distribution have mingled and the two have been originally regarded not just as a characteristic of blockchain, but probably as the fundamental characteristic defining blockchain: blockchain is decentralized (see for instance De Filippi and Wright, 2018: chapter 1). Numerous works have emerged discussing how blockchain achieves decentralization and what this means and implies in the case of decentralized technologies (Wright and De Filippi, 2015; Reyes, 2016), architectures (De Filippi, 2016), infrastructures (Glaser, 2017; Pereira et al., 2019), platforms (Pereira et al., 2019; Wright and De Filippi, 2018: chapter 1), consensus protocols and mechanisms (Glaser and Bezzenberger, 2015; Tasca et al., 2017; Werbach and Cornell, 2017), governance (Atzori, 2015; Arrunada and Garicano, 2018), money and currencies (Gervais et al., 2014; Pagnotta, 2018; Tschorsch and Sceuermann, 2016), and even regulation (Nabilou, 2019). In all these works, decentralization represents the starting point of the analysis; in other words, decentralization is usually the given preliminary assumption from which the analysis develops.

The definition of blockchain in function of the concept of decentralization creates evident conceptual, epistemological and analytical difficulties: if decentralization is the premise of the analysis, it becomes impossible (or even irrelevant) to test whether blockchain really is decentralized, or even what this means. As noted by Walch (2019a: 2) in a recent working paper, the logic becomes very similar to the argument that "blockchain system is decentralized because, well, blockchains are decentralized". Moreover, the diversity of the interpretative approaches (which depend on the point of view adopted) also generates a lack of clarity and prevents a common understanding of the concept to be tested and analysed. As decentralization is the preliminary assumption from which the argument develops, the concept is very rarely defined.

The lack of a clear and precise definition also generates other confusions and misunderstandings. One striking example is that decentralization (the newer concept used by the Ethereum founders) is commonly mixed up by the literature with the concept of distribution (the original concept used by bitcoin creators). As a result, blockchain as a decentralized technology is usually interpreted as a distributed ledger technology (DLT), even though decentralization and distribution refer to two very different things: distribution simply refers to the way in which something is dispersed, accessed or appropriated by various actors of a social system (usually resources, content or data), while decentralization is a relational concept that defines the way in which interpersonal relationships between these actors are structured, organised and governed. This same confusion is also present in

\footnotetext{
${ }^{13}$ There is just one single exception where the term decentralization appears. The full email exchange can be found at: https://satoshi.nakamotoinstitute.org/emails/cryptography/threads/1/
} 
the broader literature on digital networks and the internet where distribution and decentralization are often conflated (Mathew, 2016; Möller and von Rimscha, 2017; Troncoso et al., 2017), and in the literature on digital infrastructures, where both the term distribution and decentralization is used (Hanseth and Lyytinen, 2010; Tilson et al, 2010).

\subsubsection{Critiquing Decentralization in Blockchain Networks}

A series of events and general developments challenges the notion of decentralization, such as: 1) The hacking of Mt Gox in 2013 and its subsequent bankruptcy in 2014; 2) the development of specialised $\mathrm{ASIC}^{14}$ (Application-Specific Integrated Circuit) cryptocurrency mining equipment allowing for the emergence of powerful mining pools ${ }^{15}$ that potentially would be able to reach the critical $51 \%$ of mining capacity for a specific cryptocurrency network and through this subvert the distributed consensus; ${ }^{16}$ and 3 ) the collapse of the DAO leading to the forking of Ethereum in $2016 .{ }^{17}$ These important events showed that decentralization cannot be treated as an inherent characteristic of the blockchain technology, of its tokens, or of the social communities adopting it. In the light of these events, an increasing amount of works have recently started to question the assumption of decentralization by showing that blockchain is far from the archetype of decentralization that is usually portrayed to be.

As a result, some works have focussed on the collapse of Mt Gox and on the emergence of many other wallet providers and exchange platforms to show that the blockchain ecosystem abounds with central intermediaries that, most importantly, also represents single points of failure of the entire network (Beikverdi and Song, 2015; Gencer et al., 2018; Vidan and Lehdonvirta, 2019). The emergence of very powerful mining pools showed that the blockchain protocol could be centrally controlled by a handful and maybe even one single actor (as for Ghash.io), and it was therefore prone to manipulation regardless of the will of the majority and the incentive mechanisms adopted (Eyal and Sirer, 2018; Kwon et al., 2019; Vidan and Lehdonvirta, 2019).

The hacking and bankruptcy of the DAO and the implications that this event had for the underlying Ethereum blockchain network has fostered what is probably the most interesting and prolific strand of critiques to the assumption of decentralization. An increasing amount of recent works have focussed on the governance of blockchain networks and on their inherent problems and failures (Arrunada, 2018; Beck et al., 2018; De Filippi and Loveluck, 2016; Dupont, 2018; Finck, 2019: chapter 8; Hsieh et al., 2018; John and Pam, 2018; Miscione et al., 2018 and 2019; Musiani et al., 2018; Reijers et al., 2016; Reyes, 2019; Walch, 2015 and 2018; Zachariadis et al., 2019; Ziolkowski et al., 2019). These works have mainly compared the problems emerging from blockchain governance to the governance of digital platforms and, especially, of digital infrastructures. This literature discusses how the way in which decisions are taken, adopted, and implemented always reflects some governance arrangement of the social community that is far from the ideal of pure decentralization as portrayed by the literature in economics, management and organization studies.

\footnotetext{
${ }^{14}$ https://en.wikipedia.org/wiki/Application-specific_integrated_circuit

${ }^{15}$ The pooling of computer capacity to gain a more predictable cryptocurrency awards from proof-of-work consensus arrangements https://en.wikipedia.org/wiki/Mining_pool

${ }^{16}$ https://www.coindesk.com/bitcoin-miners-ditch-ghash-io-pool-51-attack

${ }^{17}$ https://en.wikipedia.org/wiki/Ethereum
} 
This governance conundrum is not limited to the Ethereum community. Many other blockchain communities are currently experiencing "governance crises" derived from the need to update and change their respective protocols in response to a variety of urgent and pressing issues, such as: 1) ensuring sufficient performance when scaling the network; 2) ensuring that the blockchain consensus mechanism can resist the advantages of ASIC mining equipment; 3 ) the environmental impact of cryptocurrency mining; 4) the interconnection and interoperability of different blockchain networks, and 5) the privacy in exchange of tokens (for a similar list of challenges, see Zachariadis et al., 2019). This has fostered an emerging fierce debate over the meaning and the nature of blockchain governance much beyond academia, and even among the various members of these communities (Buterin, 2017b and 2018). ${ }^{18}$ These debates on blockchain governance among members of the blockchain communities inevitable mingled with, and result into, the debate on the nature and meaning of de/centralization in blockchain networks (Halaburda and Mueller-Bloch, 2019; Srinivasan and Lee, 2017). ${ }^{19}$

\subsection{Problems with the Current Literature}

Although these recent works do not up front assume that blockchain by assumption is a decentralized system, there are still fundamental problems with the current literature discussing the centralizing trends of blockchain networks. First, all works critically challenging the assumption of decentralization usually adopt a partial and mono-dimensional approach to decentralization by focussing on one specific aspect or dimension of blockchain technology, thus ignoring the multidimensionality of blockchain and the related inter-disciplinary nature of the concept of decentralization (Schneider, 2003 and 2019). By doing so these works mainly provide narrow and tailor-made definitions of decentralization for the very specific case of blockchain (or digital networks at most), while ignoring the fact that decentralization is a much more fundamental characteristic of any social (and socio-technical) system where actors and individuals interact in order to pursue their interests and enact their will in relationship (and often in conflict) with the interests and will of others. Secondly, these works usually fail to provide an analytical definition of decentralization, while they mainly focus on its antonym, centralization. The goal is usually to argue that blockchain is not decentralized by showing and verifying that blockchain networks present centralizing tendencies. But what centralization and, especially, decentralization precisely mean and how they should be conceptualised has so far remained vague, implicit, or completely unaddressed (Walch, 2019a). Let us analyse these two aspects in turn.

\subsubsection{Decentralization as a Lack of Centralization}

Even though many works now recognise that blockchain networks manifest centralizing tendencies, each work interprets centralization according to the interpretative lens provided by its own specific epistemological field and, as a result, each work only focuses on one single aspect of de/centralization. At least four interpretative approaches to de/centralization can be inferred from the literature (see also Buterin, 2017a; Schneider, 2003 and 2019 on this issue): a technological (or

\footnotetext{
${ }^{18}$ This is particularly evident in the Ethereum community, where prominent members are discussing the way in which blockchain governance can be applied to the Ethereum blockchain. For a summary of the debate, see the overviews provided by CleanApp on Medium (CleanApp, 2018a, 2018b, 2018c, 2019).

${ }^{19}$ See also the approach adopted by ConsenSys to measure centralization in ethereum: https://consensys.net/research/measuring-blockchain-decentralization/
} 
infrastructural) one, an economic one, a political one, and a legal one (see table 2.1 for a summary of the four approaches)

\section{a. Architectural and logical approaches: technological centralization of networks}

First, a technological or infrastructural approach to centralization defines centralization by looking at the technological characteristics of a networked system and at the way in which the network is designed. Following Buterin (2017a), two different variations of this technological or infrastructural approach can be identified.

The architectural approach defines centralization as a degree of redundancy of the system (Baran, 1964: 1). For this reason, architectural decentralization can be intended as a measure of the number of nodes that can break down before the entire system breaks down (Buterin, 2017a). In this case a networked system is defined as centralized if the network presents a central point of failure, or bottleneck, in the form of an intermediate or focal node collecting, aggregating, organising, and rooting the traffic generated by other nodes. The architectural approach defines centralization as a function of the design of the network's linkages. We define this approach as architectural as it focusses on the architecture of the network intended as the design of the network's pipes and conduits connecting the various nodes.

The works adopting an architectural perspective mainly focus on the role played by big wallet providers and exchanges and acquired an early prominence in the literature following the rise (and fall) of big exchanges (such as Mt.Gox). The architectural approach is also widely adopted by the managerial literature on platforms' architecture and ecosystems (Baldwin and Woodward, 2009; Langlois and Robertson, 1992), by the economic literature on networks (Economides, 1996; Shapiro \& Varian, 1998; Shy, 2001), and by the sociological literature (Burt, 1992; Granovetter, 1973 \& 1985; Powell, 1990; Smith-Doher and Powell, 2005; Uzzi, 1997). In all these approaches the focus is on the structure or architecture of the relationships between the elements (nodes).

The second sub-branch of the technological-infrastructural approach to centralization is represented by what can be called the logical interpretation of centralization (Buterin, 2017a). This second approach is the one more closely rooted in the computer science literature and is the one directly linked to the theory of distributed or peer-to-peer (p2p) systems (Tanenbaum and van Steen, 2007). In this case, centralization is still a static feature of the infrastructure, but the focus is shifted from the architecture of the pipes "in the middle" to the role of the nodes "at the edges". A networked system in this case is logically centralized if the various nodes within the system behave as a unique and coherent entity, and cannot operate autonomously and independent from each other as this would breach the integrity and consistency of the system. Logical centralization implies some form of social coordination or collaboration, while the various elements in a logically decentralized system are potentially adversarial and might act inconsistently from each other (Troncoso et al., 2017).

Logical centralization simply requires that all nodes are on the same page and share the same common vision of the world and of the state of the system so that they can cooperate and collaborate towards a common and coherent goal. Logical centralization implies an agreement among the nodes on some common state or goal. For this reason, some shared resource is necessary to retain the logical consistency and integrity of the overall system across all nodes as this facilitates the social coordination by ensuring the alignment of incentives. Obviously, this integrity can be ensured by means of centralized architecture through a shared access to a common intermediate 
hub, but it does not need to. Coordination can simply be ensured by some form of logical centralization by ensuring sharing of information, or alignment of incentives or goals. For this reason, the focus of logical centralization is on the edges (the way in which common resources or data are distributed to, or shared by, the edges), not on the pipes (the way in which these data and resources can be transmitted and communicated).

Peer-to-peer ( $\mathrm{p} 2 \mathrm{p}$ ) systems can be logically decentralised and architecturally centralised in the case of p2p platforms or marketplaces such as Uber or Airbnb (Sundararajan, 2013), or more often architecturally decentralized but logically centralized, as in the case of standard distributed computer networks (Tanenbaum and van Steen, 2007). More generally, distributed systems are usually characterised by the architectural decentralization of the infrastructure but by its logical centralization. A distributed system is defined by Tanenbaum and van Steen (2007: 2) as "a collection of independent computers that appears to its users as a single coherent system." Consistent with many other p2p networks, blockchains are always logically centralized (Buterin, 2017a), where the common resources shared by all nodes and ensuring the integrity and consistency of the overall system is time or, more specifically, the blockchain timestamp. The presence of a peerto-peer distributed timestamp server ensures that all nodes synchronise transactions in the same chronological order and this ensures that a single version of a ledger is distributed among all nodes: the distribution of the timestamp server implies centralization of the system. As a result, a blockchain is logical centralized, which ensures the integrity of the system and its behaviour as a single coherent system even in the absence of architectural centralization. ${ }^{20}$

The inherent centralization brought about by a distributed timestamp has not been sufficiently highlighted by the blockchain literature on blockchain and it has been discussed only indirectly with respect to the debates on the scalability problem of proof-of-work protocols, and the need for independent side chains (Croman et al., 2016; Gervais et al., 2016). In fact, decentralization and scaling have usually been understood by industry participants as potentially incompatible aspects of a distributed system such as blockchain. ${ }^{21}$

More generally, it can be argued that it is the confusion of the logical aspect of a network (behaviour at the edges) with its pure architectural aspect (the organization of the pipes) that generates the

\footnotetext{
${ }^{20}$ The trade-off between logical and architectural de/centralization is well summarised by Nakamoto (2008: 2) in his discussion of the double-spending problem: "The problem of course is the payee can't verify that one of the owners did not double-spend the coin. A common solution is to introduce a trusted central authority, or mint, that checks every transaction for double spending ... The problem with this solution is that the fate of the entire money system depends on the company running the mint, with every transaction having to go through them, just like a bank [architectural centralization]. We need a way for the payee to know that the previous owners did not sign any earlier transactions ... To accomplish this without a trusted party, transactions must be publicly announced, and we need a system for participants to agree on a single history of the order in which they were received [logical centralization]." In sum, blockchain addresses the problems emerging from architectural centralization by introducing logical centralization and this is practically achieved by switching from point-to-point communication to broadcasting communication.

${ }^{21}$ For an overview of Buterin's scalability trilemma, see Buterin (2014b), and the overviews and summaries in Medium: https://medium.com/@aakash 13214/the-scalability-trilemma-in-blockchain-75fb57f646df ; https://medium.com/logos-network/everything-you-know-about-the-scalability-trilemma-is-probably-wrongbc4f4b7a7ef; and Coindesk: https://www.coindesk.com/decentralization-vs-scale-studies-explore-cryptosgrowing-struggle.
} 
confusing conflation of distribution with decentralization (see Troncoso et al., 2017). In Baran's (1964) famous article, the distributed system lacks architectural centralization, even though a high degree of redundancy is precisely the way in which coherence and integrity of the overall system (logical centralization) can be ensured. A broad range of literature primarily conceives of centralization in terms of its logical dimension. The focus is on the set of common rules and principles shared by all participants ensuring cooperation and coordination among various independent elements (or nodes). This is the case for works on: Social (or peer) production (Benkler, 2006), open innovation (West and Bogers, 2014), modularity (Baldwin and Clark, 2000; Langlois, 2003), and platform ecosystems (Adner, 2017; Jacobidies et al., 2018) and, especially, the one dealing with digital infrastructures and the birth of internet governance (Ciborra et al., 2000; Goldsmith and Yoo, 2006; Tilson et al, 2010).

Although it is possible to keep architectural and logical approaches distinct as done by Buterin (2017a), they both focus on some objective design features of the network's infrastructure and for this reason we think that it is possible to consolidate them into one single common approach: the technological or infrastructural approach to centralization (this is similar to Scheneider (2019: 15-17) network approach).

Buterin (2017a) completes his taxonomy with a last dimension, political centralization, defining the extent to which different individuals controls the various nodes of the network. This political dimension is also highlighted by Scheneider (2019). However, control can emerge in various ways and for different reasons, making it very hard to group all the social determinants of centralization into one single dimension. As a result, three other approaches can be identified from the literature.

\section{b. Economic approaches to centralization}

A second approach to centralization shared by many works discussing centralization in blockchain networks can be defined as the economic interpretative approach. In this case authors criticising the decentralization assumption of a blockchain highlight how a handful of mining nodes can control the entire system by escalating their investments and concentrating the mining power (Kwon et al., 2019). This line of research mainly considers the dispersion of computing power intended as a proxy for the capacity of one or few nodes to unilaterally affect the way in which the network can evolve and behave. This can be defined as an economic approach as the dynamics according to which the processing capacity of the various mining nodes tend to coalesce and be concentrated into, and thus controlled by, a few or many nodes is usually studied through standard game-theoretic models of market dynamics: degree of competition, incentives to invest, market concentration, collusion and monopoly power (Abadi and Brunnermeier, 2018; Biais et al., 2019; Chiu et al., 2017; Huberman et al., 2017; Kwon et al., 2019; Ma et al., 2018). Consistent with the economic approach, these works adopt a social welfare perspective on centralization.

While the architectural critique of decentralization was inspired by the emergence and failure of central intermediaries, the economic approach to centralization was clearly inspired by the centralization of hashing power (computing power) into few big mining pools (such as BTC.com or Antpool) following the development of ASCl appliances in 2011-12. This aspect became especially relevant considering that the biggest manufacturer of consumer appliances (Bitmain) is also the one directly managing one of the biggest mining pools (AntPool). 


\section{c. Political approaches to centralization}

A third approach considers political de/centralization. The degree of political centralization is usually interpreted by the literature as the way in which private preferences, or wills of single actors, are aggregated and represented at the public (or social) level (Torrisi et al., 2011: 10). Political decentralization measures and defines the rules governing participation to, and representation in, the public sphere: "The best way to summarize these functions might be in terms of representation, which refers to the way political institutions map the multiplicity of citizen interests onto policy decisions [...]" (Schneider, 2003: 39). The political approach to decentralization characterises the degree of centralization of the governance of a social system, or the process through which collective decisions are taken.

The political approach usually looks at centralization in terms of normative political categories, such as democracy or fairness. As the political approach focusses on the way in which private values are aggregated, weighted, and translated into public values, it is inherently evaluative and normative (Schneider, 2003). In this case, decentralized systems are democratic systems where all actors have the same "weight" and social influence, and therefore the same probability to see their instances represented and embraced. In this case, a centralized system becomes a hierarchy whenever few actors can take decisions in behalf of the others, such as in an oligarchy, or when the decisions taken by few actors weights more than the will and preferences of others, as in aristocracy or plutocracy.

The political approach emerged fairly recently, following the governance problems characterising many blockchain networks, especially with respect to the responses to hacking, protocol change, and scalability. This approach is the one mainly followed by the works interested in the way in which blockchain communities govern themselves, and on the way in which rules governing blockchain networks are developed and established by their respective communities, especially with respect to the proof-of-stake consensus protocol (Buterin, 2017b; 2018). ${ }^{22}$ The focus is mainly on the various governance forms, such as hierarchy, market, network, and bazaar (see also Ziolkovski et al., 2018a and 2018b; Miscione, 2019). More generally, the literature analysing blockchain governance and the debates emerging out of it mainly adopt a political understanding of de/centralization. A similar approach is also adopted by the literature on open access and networked-production that compares network governance arrangements to hierarchies. Thus, Benkler (2006: 62) defines a system as decentralized whenever coordination can happen without hierarchy, and when "many agents cohere and are effective despite the fact that they do not rely on reducing the number of people whose will counts to direct effective action". This is a political interpretation of decentralization (democracy) nested within a requirement of logical centralization (overall coherence and effectiveness of the system).

\section{d. Legal approaches to centralization}

The legal interpretation of centralization is a fourth and last to be found in the literature. This approach is similar to, yet distinct from, the political approach as it mainly looks at the legal dimension of centralization. A system is centralized whenever duties, obligations, responsibilities, accountabilities and liabilities can be clearly identified and clearly attributed to some actor. Decentralization in this case implies duties and liabilities equally shared or diffused among all

\footnotetext{
${ }^{22}$ See also Morris at https://breakermag.com/vitalik-buterin-is-embracing-a-new-role-political-theorist/
} 
members, thus generating confusion with respect to who is responsible for what or impossibility to trace responsibilities back to a specific actor or a defined set of actors. Systems are legally decentralized whenever it is not possible to identify specific actors bearing special liabilities, duties and responsibilities vis-à-vis the others - claimants.

This approach is closely linked to the political approach as it still focusses on the governance dimension of a blockchain, but also differs as it focuses on liabilities and responsibilities rather than on the relative "weight" of the various actors playing a role in the decision process and shaping the final outcome (even though the political and legal aspects may be strictly linked as concentration of political power often implies concentration of legal responsibility and liability and vice versa; see $\emptyset$ stbye, 2019 and Zetzsche et al., 2018). At the same time, if the political approach focusses on the governance issues of the blockchain intended as hierarchies in the decision-making process, the legal approach is mainly interested in blockchain as corporate governance intended as a principal-agent problem between managers (administrators or validators) and owners (coin holders), sometimes also reflecting a coordination problem between on-chain and off-chain interests respectively (Finck, 2019: 191-206; Reijers et al., 2018; Reyes, 2019; Walch, 2019; Yermack, 2017). Decision rights and responsibilities are often treated jointly as two separate aspects of blockchain governance (Beck et al. 2018).

The recent problems faced by regulators with respect to the issue of crypto-tokens and Initial Coin Offerings (ICOs) are mainly rooted in this fourth approach (Van Valkenburgh, 2018a \& 2018b). Legal centralization becomes particularly relevant in the case of hacks, scams, frauds, or damages where liabilities, duties and criminal responsibilities must be clearly identified, even in the case of smart contracts (Finck, 2019: 58-64; Werbach, 2018; Zetzsche et al., 2017). More generally, this legal approach to decentralization is particularly relevant in the case of autonomous systems, open source software and distributed communities. Table 2.1 summarizes the four approaches.

\begin{tabular}{|l|l|l|l|}
\hline \multicolumn{1}{|l|}{ Interpretative Approach } & \multicolumn{1}{|c|}{ Definition of centralization } & \multicolumn{1}{|c|}{$\begin{array}{c}\text { Aspect of blockchain interested by the } \\
\text { approach }\end{array}$} \\
\hline $\begin{array}{l}\text { Infrastructural } \\
\text { Infochofical }\end{array}$ & Architectural & $\begin{array}{l}\text { Presence of central intermediaries or } \\
\text { bottlenecks (lack of redundancy in the } \\
\text { linkages) }\end{array}$ & $\begin{array}{l}\text { Emergence of big wallet providers or crypto } \\
\text { exchanges marketplaces serving lightweight } \\
\text { nodes representing single points of failure }\end{array}$ \\
\cline { 2 - 4 } & Logical & $\begin{array}{l}\text { Presence of some shared resource / logic } \\
\text { / incentives allowing for coherence and } \\
\text { integrity of the overall system, which } \\
\text { appears as a unique and coherent entity }\end{array}$ & $\begin{array}{l}\text { The essence of distributed systems such as } \\
\text { blockchain: Distributed ledger and shared } \\
\text { access to the same database }\end{array}$ \\
\hline Economic & $\begin{array}{l}\text { Accumulation of capabilities in single } \\
\text { nodes generating concentration of power } \\
\text { (market power) as an ability to } \\
\text { unilaterally influence the system's } \\
\text { behaviour }\end{array}$ & $\begin{array}{l}\text { Development of crypto-specific } \text { ASIC mining } \\
\text { hardware Emergence of big mining pools } \\
\text { and mining farms centralizing hashing power }\end{array}$ \\
\hline Political & $\begin{array}{l}\text { Presence of special status individuals } \\
\text { whose will and decisions weight or count } \\
\text { more than others' when deriving } \\
\text { collective decisions on how the system } \\
\text { ought to behave }\end{array}$ & $\begin{array}{l}\text { Governance problems emerging from } \\
\text { problems requiring code upgrade / change } \\
\text { (hacking of DAO, scalability, ASIC resistance, } \\
\text { interoperability) }\end{array}$ \\
\hline Legal & $\begin{array}{l}\text { Possibility to clearly attribute duties, } \\
\text { obligations, responsibilities and liabilities } \\
\text { to specific actors / possibility to held } \\
\text { specific actors accountable }\end{array}$ & $\begin{array}{l}\text { Corporate governance problems emerging } \\
\text { from the sale of tokens and services over the } \\
\text { blockchain (ICOs) or from faulty code }\end{array}$ \\
\hline
\end{tabular}

Table 2.1: Four interpretative approaches to de/centralization of centralization identified in the current blockchain literature. 
As summarized in table 2.1, different approaches focus on different aspects of digital networks and, for this reason, they provide a different (yet complementary) understanding of de/centralization. As each captures a partial approach, all play a part, no single approach is sufficient in itself to provide an exhaustive understanding of de/centralization. The technological approach focusses on the way in which a socio-technical network is structured and operated. On the other hand, the economic approach focusses on the way in which on-chain consensus is reached and decisions are validated by the network's nodes. The political approach can either focus on on-chain governance in case decisions are taken on-chain, or on the off-chain dimension of governance, in case the decisionmaking process is undertaken off-chain. Finally, the legal approach defines de/centralization by looking at how decisions are linked to responsibilities, rights, duties and liabilities.

In conclusion, although the recent emergence of such a rich and diverse scope of works overcomes the fundamental problems deriving from the assumption of decentralization in blockchain networks, they still reproduce a fundamental problem of the earlier works: by focussing on one single dimension, these "disciplinary" works can only capture single aspects of the technology, rather than the whole picture. However, decentralization is a multi-dimensional concept acquiring its meaning from the dynamic interaction and variable combination of its various dimensions. As argued by Schneider, 2003: 40), "What has not yet been discussed, but remains pertinent, is the degree to which these dimensions are interrelated. This interrelationship can contribute to conceptual confusion, as decentralization along one dimension may influence or cross over into decentralization along another dimension. Without accounting for this interrelationship, measures will over- or underestimate the amount of decentralization" (see also Schneider, 2019).

\subsubsection{Defining a De/centralization Framework}

The current blockchain literature rarely provides an operational definition of decentralization. The majority of the works criticising the decentralized ideology of blockchain are primarily descriptive endeavours that simply "verify" that blockchain networks in reality manifest some centralizing tendencies. Very often a lack of decentralization is indirectly inferred from the verification of some form of centralization. This generates two main problems.

First, centralization lacks a univocal meaning and is implicitly defined in various ways, depending on the epistemological approach adopted. Centralization is sometimes implicitly understood as denoting the presence of some form of power (Walch, 2019a). However, power can either have a socio-political origin (the political approach), an architectural nature (the approach adopted by the sociology of networks), or it can derive from actors' inherent capabilities shaping the resulting market structure in which these actors operate (the economic approach). Other times centralization is defined in terms of control, for example over other actors or resources: (Schneider, 2003). This interpretation mainly belongs to the economic and political approach and is especially adopted by the managerial and organizational literature dealing with the governance of platforms and infrastructures (Gawer, 2014; Tilson et al, 2010; Constantinides et al, 2018) and of digital networks in general (Zittrain, 2008). In the case of blockchain, centralization as control is mainly relevant in those works inquiring the governance and corporate governance of blockchain networks. Yet, the definition of centralization as control rarely emerges from the technological approach. Other times centralization is defined in terms of accumulation and appropriation and therefore in terms of property rights. The economic and logical approaches are the ones mainly adopting this interpretation: the focus in this case is mainly on the nature and amount of resources that the 
various nodes can accumulate. The managerial literature reframes this problem as an access problem, where a lack of access derives from the impossibility to appropriate someone else's property. Finally, the legal approach defines centralization in terms of rights and duties, but not necessarily as property rights. Although the concepts of power, right, and control are intimately linked, they remain distinct concepts meaning different things, thus capturing different, interlinked, aspects or phenomena.

The second problem derives from the fact that verifying that blockchain manifests clear tendencies towards centralization does not mean that the term decentralization is clearly defined, or that an operational framework to test and understand decentralization is provided. The definition of centralization is often somewhat easier than the one of decentralization. As stated by Schneider (2003: 34), "Researchers have multiplied the conceptualizations of decentralization; associated the various concepts with different meanings ... By way of contrast, decentralization's antonym, centralization, has a much more precise and accepted usage, which is the concentration of power, resources, and authority in a single head or center." This is true also for the various works challenging the decentralization ideal of blockchain: a common aspect to all these works is the treatment of centralization as a form of concentration.

The few works providing operational approaches usually measure the degree of centralization by looking at some measure of concentration, rather than defining decentralization itself (Kwon et al., 2019; Srinivasan, 2017). This can be reasonable in some cases, but is not necessarily exhaustive in others. While appropriation and property rights nearly always generate some type of concentration, social power and rights do not necessarily manifest themselves in terms of concentration. In this case, the measure of qualitative or immaterial imbalances or inequalities might become more appropriate. Most importantly, it is not always entirely clear how decentralization should be defined if expressed and intended as a lack of centralization. What does complete lack of power, control, or rights mean? What does it mean for a complex and multidimensional social system such as a blockchain to be completely decentralized? Based on Hohfeld's $(1913 ; 1917)$ fundamental legal taxonomy, it is possible to summarise the various interpretations of centralization provided by the literature as in table $2.2{ }^{23}$ Table 2.2 provides an attempt to link the generic legal categories to the four aforementioned epistemological approaches to de/centralization.

With the exception of a handful of very recent works and articles (Arrunada, 2018; Schneider 2019; Troncoso et al., 2017; Walch, 2019a), the majority of the works do not critically enquire the concept of decentralization by asking what decentralization means in the context of blockchain, how it should be defined and conceptualised, and how it relates to or emerges from centralization. Even among the ones that do critically examine the meaning of decentralization in very general terms (Walch (2019a) defines centralization as "power"), very few adopt a comprehensive and multidimensional approach (Schneider 2019 is an example).

\footnotetext{
${ }^{23}$ Hohfeld (1913) distinguishes between rights, powers, privileges and immunities and their jural opposites. Differences in privileges and immunities among actors might be factors explaining centralization (differences in mining capabilities confer control over the network, but they are de facto differences in privileges, in Hohfeld terminology), but they are rarely used in the literature to define and characterise de/centralization.
} 


\begin{tabular}{|l|l|l|l|}
\hline $\begin{array}{l}\text { Centralization } \\
\text { intended as: }\end{array}$ & \multicolumn{1}{|c|}{ Meaning } & \multicolumn{1}{|c|}{ Applied to Blockchain } & \multicolumn{1}{|c|}{$\begin{array}{c}\text { Primary } \\
\text { Epistemological } \\
\text { Approach }\end{array}$} \\
\hline Power & $\begin{array}{l}\text { Ability to affect, influence and } \\
\text { modify the social status of others } \\
\text { or the state of a thing }\end{array}$ & $\begin{array}{l}\text { Centralization of cryptocurrency } \\
\text { mining power, of coding } \\
\text { capabilities and decision rights }\end{array}$ & $\begin{array}{l}\text { Economic } \\
\text { Political } \\
\text { Architectural }\end{array}$ \\
\hline $\begin{array}{l}\text { Control } \\
\text { (privileges or } \\
\text { capabilities) }\end{array}$ & $\begin{array}{l}\text { Ability to determine or command } \\
\text { how a thing or actor should behave } \\
\text { at will }\end{array}$ & $\begin{array}{l}\text { Centralization of mining power, } \\
\text { hierarchical governance and } \\
\text { corporate governance of } \\
\text { blockchain }\end{array}$ & $\begin{array}{l}\text { Economic } \\
\text { Political } \\
\text { Legal } \\
\text { Architectural }\end{array}$ \\
\hline Rights & $\begin{array}{l}\text { Legal capacity to limit or constraint } \\
\text { the behaviour of others (by } \\
\text { constraining the set of available } \\
\text { options or opportunities of duty } \\
\text { holders) }\end{array}$ & $\begin{array}{l}\text { Centralization of duties, } \\
\text { obligations, responsibilities, } \\
\text { accountabilities and liabilities in } \\
\text { the corporate governance of } \\
\text { blockchain }\end{array}$ & $\begin{array}{l}\text { Legal } \\
\text { Political }\end{array}$ \\
\hline Property rights & $\begin{array}{l}\text { Very specific type of constraint or } \\
\text { limitation: Legal capacity to exclude } \\
\text { others from interacting with } \\
\text { something (access or use) }\end{array}$ & $\begin{array}{l}\text { Centralization of mining } \\
\text { through escalation of } \\
\text { investment and appropriation } \\
\text { of assets; centralization as } \\
\text { private access to data and } \\
\text { information (no shared } \\
\text { database) }\end{array}$ & $\begin{array}{l}\text { Economic } \\
\text { Political }\end{array}$ \\
\hline
\end{tabular}

Table 2.2: Four epistemologies of centralization based on Hohfeld's $(1913 ; 1917)$ fundamental legal taxonomy and applied to blockchain

\subsubsection{Defining de/centralization: a framework for the study of socio-technical system}

There is arguably a common trait shared by all interpretative approaches to de/centralization: they all recognise that centralization and decentralization are social and inter-personal categories, in the sense that they only make sense whenever two or more actors (individuals, groups or organizations) inter-act and affect each other in some manner. Actions that are inter-personal (take place between persons) become inter-actions. This is also the basic role of any network (no matter whether in its platform or infrastructural form): to enable inter-action between different actors otherwise isolated and independent from each other (Hanseth and Lyytinen 2010; Henfridsson and Bygstad 2013). As recognised by the literature, platforms and infrastructures are also social entities or, more precisely, socio-technical artefacts (de Reuver et al, 2018).

Inter-personal actions, or interactions, differ from actions done in isolation or autonomy in that they always create some form of inter-dependency. A mutual dependency between two or more actors, to be contrasted to the in-dependence characterising autonomous actions of isolated actors. Here, we critically characterise the centralization-decentralization dichotomy in terms of interdependence. There is interdependence whenever what can be achieved by one member of a social system depends more or less directly, and in a more or less conscious or purposeful way on the decisions/actions/will of others. Centralization might emerge from decentralization and vice versa, depending on the nature of the interdependencies among the actors.

The work defines any social system composed by two or more members as "centralized" whenever there is an asymmetric ${ }^{24}$ interdependence between them. By contrast, we define decentralization as

\footnotetext{
${ }^{24}$ The concepts of symmetry and asymmetry has been applied across a range of academic fields for particular purposes as well as being studied as a foundational concept for our understanding of the Universe (Weil, 1983; Close, 2001; Stewart, 2007). Here, we apply the concept in a context of social systems in the context of interactions and relationships that can be externally agreed, as for example, opposed to the social-
} 
symmetric interdependence between actors. The interdependence can be symmetric if two members are able to influence each other in the same way as they are able to sort the same effects on the others whenever they take the same course of action. Symmetry defines a situation where no member can affect the other more than the counterpart can. Interdependence becomes asymmetric whenever this is not true, meaning that one member's fate is more dependent on the decisions and actions of others than these are dependent on his or her. Asymmetric inter-dependence can also be intended as dependence of one member on the other, in the sense that the former's fate depends on the decisions, choices and actions of the latter.

As a result, we argue that perfect decentralization occurs in two ideal cases: absence of interdependence among members tout court (autonomy or freedom), and perfect symmetry in interdependency (absence of dependency). In both cases, perfect decentralization is characterised by equality of all the members vis-à-vis each other. A social system is characterised by some degree of centralization whenever there is interdependency among their members, and this interdependence presents some degree of asymmetry ("interdependence between" becomes "dependence on"). The degree of asymmetry defines the degree of dependence, which can be taken as a measure of the degree of centralization in a social system. This degree of asymmetry also characterises the level of inequality among the various members of a social system. Decentralization, symmetry, and equality all describe the same state of affairs in the same way in which centralization, asymmetry, and inequality do. The extreme case where one single individual always decides on behalf of everyone else represents the maximum degree of degree of inequality, and therefore also the maximum degree of centralization. The same concepts can be formalised according to Hohfeld's $(1913 ; 1917)$ fundamental legal taxonomy. First, when interconnection is absent, actors, which can be single individuals, organizations or collectives depending on the unit of analysis, operate in isolation and complete autonomy. This state corresponds to the a-social state of nature where interdependence is inexistent. In Hohfeld's terms (1913), a system where actors are independent is characterised by sole liberties or privileges. As perfect independence rules out any type of interdependence between the actors, the system can be treated as a perfectly decentralized one. As soon as socio-technical artefacts such as infrastructures or platforms are built, some form of interconnection between previously independent actors is enabled and interactions might appear. With interconnections and interactions, interdependencies emerge (Grisot et al., 2014; Hanseth et al., 1996; Hanseth and Lyytinen, 2010; Henfridsson and Bygstad 2013). These interdependencies can maintain perfect decentralization or generate centralization and can be de facto (emerging informally from actors' abilities, opportunities of faculties) or de jure (formalised in social rules)

Social systems are de facto perfectly decentralized whenever they are characterised by completely symmetric set of abilities, capabilities or opportunities. Whenever this is not the case, perfect decentralization of a social system might be established de jure, by completely symmetric set of rights, which define symmetric reciprocal possibilities and duties among all actors. Otherwise, social systems can present some degree of centralization de facto, due to asymmetries in capabilities, opportunities, abilities or faculties among the actors. In Hohfeld's (1913) terminology, these centralized systems are characterised by power of some members on others (power relations are

psychological aspects relating an inner individual psychological state to the outer social world, as for example explored by Goffman (1959). 
always asymmetric, and can be de facto). Alternatively, asymmetries can be formalised in asymmetric rights among members (asymmetries de jure). Asymmetric rights also imply social power and control over other members' behaviour (thus hierarchies) if referred to other individuals (rights in personam) or control over things (asymmetric rights in rem emerging from property rights exclusion and appropriation of things).

Table 2.3 summarises our categorization of centralization and decentralization.

\begin{tabular}{|c|c|c|c|c|c|}
\hline Category & $\begin{array}{l}\text { Precondition, } \\
\text { characteristic of } \\
\text { the situation }\end{array}$ & Definition provided & $\begin{array}{c}\text { Normative } \\
\text { Political } \\
\text { categories }\end{array}$ & & $\begin{array}{l}\text { Hohfeld positive legal } \\
\text { categories }\end{array}$ \\
\hline \multirow{3}{*}{$\begin{array}{l}\text { Perfect } \\
\text { Decentralization }\end{array}$} & A-social actions & Independence & Liberty & De facto & Capabilities / privileges \\
\hline & \multirow[t]{2}{*}{$\begin{array}{l}\text { Social } \\
\text { inter-actions }\end{array}$} & \multirow[t]{2}{*}{$\begin{array}{l}\text { Symmetric } \\
\text { interdependence }\end{array}$} & \multirow[t]{2}{*}{ Equality } & De facto & $\begin{array}{l}\text { Immunities / disabilities: } \\
\text { Symmetric capabilities / } \\
\text { opportunities / faculties } \\
\text { (absence of power } \\
\text { relations) }\end{array}$ \\
\hline & & & & De jure & $\begin{array}{l}\text { Symmetric rights (\& } \\
\text { duties) }\end{array}$ \\
\hline \multirow[b]{2}{*}{ Centralization } & \multirow[b]{2}{*}{$\begin{array}{l}\text { Social } \\
\text { inter-actions }\end{array}$} & \multirow[b]{2}{*}{$\begin{array}{l}\text { Dependence } \\
\text { (Asymmetric } \\
\text { interdependence) }\end{array}$} & \multirow[b]{2}{*}{ Inequality } & De Facto & $\begin{array}{l}\text { Power relations } \\
\text { (Asymmetric capabilities } \\
\text { / opportunities / } \\
\text { faculties) }\end{array}$ \\
\hline & & & & De Jure & $\begin{array}{l}\text { Asymmetric rights } \\
\text { In personam: Control } \\
\text { over other members and } \\
\text { hierarchies (power de } \\
\text { jure) } \\
\text { In rem: control over } \\
\text { things (property rights) }\end{array}$ \\
\hline
\end{tabular}

Table 2.3: A summary of the categories of centralization and decentralization.

As shown in Table 2.3, by defining centralization and decentralization in terms of independence, symmetric interdependence, and dependence (asymmetric interdependence), it is possible to provide a comprehensive and coherent framework for the study of de/centralization that unifies all disjointed classifications and definition provided by the literature and provides some epistemological and analytical clarity. The role played by power, control, rights, and property rights can be completely characterised and derived by simply looking at the sources and nature of asymmetries.

In summary, we argue that centralization and decentralization do not denote given and static characteristics of single given dimensions of a socio-technical system, such as the design of its architecture or the logic of its transmission protocols. Conversely, the framing of de/centralization in terms of asymmetries shows that a system can be either centralized or decentralized depending on the changing nature of the relationships and interactions between its members and elements in general. These relationships can be between elements of a single dimension or, most importantly, across dimensions. If this framework has to be applied to the blockchain, it is therefore first necessary to understand what the core elements of a blockchain are in relation to its various dimensions. In order to do so, it is necessary to understand how the platform and the infrastructural nature of any digital network (thus of blockchain as well) can be understood and interact. 


\section{A Digital Network De/centralization Framework}

The purpose of this section is fourfold with a subsection covering each: 1) Identify the deficiency of the literature to theoretically define blockchain in terms of platform and infrastructure, 2) identify the fundamental characteristics of digital platforms and infrastructures; 2) develop a conceptual framework for the study and understanding of digital networks in function of the key dimensions of platforms and infrastructures, and 3) provide a conceptual framework for the analytical study of the meaning of de/centralization in digital networks in function of the fundamental characteristics of digital platforms and infrastructures.

\subsection{Blockchain Decentralization: Literature Overview}

A third important shortcoming emerges from the literature examining the centralizing trends of blockchain technologies. No work makes any attempt to link the discussion on the degree of centralization of blockchain to the properties of platforms and infrastructures. A blockchain is first and foremost a digital network and it should be studied and analysed as such. Various works provide in-depth taxonomies of blockchain and provide extensive overviews of all their properties qua digital networks, but they are mainly descriptive works, and not really critical analyses of how specific features and dimensions of a digital network affect the nature and degree of de/centralization of a blockchain (for examples of such descriptive works, see for example: Ballandies et al., 2018; De Rossi et al., 2019; Okada et al., 2017; Rausch et al., 2018; Risius and Spohrer, 2017; Tasca and Tessone, 2017; Tasca, Thanabalasingham and Tessone, 2017; Walsh et al., 2016; Xu et al., 2017). Some works rooted in the computer science literature discuss problems or failures deriving from the centralization of some layers of the blockchain network (See, for example, Gervais et al., 2016; Neudecker and Hartenstein, 2018). However, these do not critically discuss the concept of decentralization and its meaning for blockchain. In fact, they often defined a blockchain protocol as "decentralized" by assumption (see also Kwon et al., 2019).

We have not been able to identify any work attempting to establish a relationship between the inherent tensions generated by the interplay between the platform and the infrastructural dimension of any digital network and the tensions emerging from the centralizing forces in blockchain networks. Neither have we identified any work attempting to study the centralizing trends of blockchain networks in the light of the platform-infrastructure interplay even though an emerging literature in media studies (Helmond, 2015; Helmond et al., 2019; Nechusthai, 2018; Plantin et al., 2018; Plantin and Punathambekar, 2019), information system (Bygstad and Hanseth, 2018; Costantinides et al., 2018; Lorenz and Stefan, 2018; Kazan et al, 2018) and legal studies (Zittrain, 2018; Flew, 2019; Flew et al., 2019) is beginning to address the issue.

Blockchain is usually defined in the literature either as a platform (De Filippi and Wright, 2018: chapter 1; Glaser et al., 2019; Mattila and Seppälä, 2018; Perreira et al, 2019), or as an infrastructure (De Filippi and Wright, 2018: chapter 2; Finck, 2019: 11). The confusion might reflect the shift in attention from the original bitcoin use case, which tend to understand blockchain as an infrastructure as also originally envisioned (Nakamoto, 2008), to the rise of the new Ethereum-based use cases supported by general-purpose networks such as Hyperledger or Corda, which are often referred to as "platforms" (Lacity, 2018). But no work has so far attempted to define blockchain theoretically in function of the concepts of digital platform and infrastructure, and through this derive its necessary and sufficient characteristics in an analytical manner. Such a study of the 
fundamental characteristics of digital platforms and infrastructures, through the case of blockchain, will also in turn enable a theoretical discussion of the degree of de/centralization of a given blockchain in terms of the infrastructural and platform aspects of a digital network.

\subsection{Defining the Access and Use Dimensions}

Even though platforms and infrastructures are two very different concepts, the two share some common traits. This makes it possible to identify some fundamental dimensions for the analysis of any digital network. The literature has recognised that both platforms and infrastructures have a dual nature and can be understood along two different dimensions: the one of access defining the scale of actors that can participate or interconnect to the network, and the one of use defining the scope of functionalities, services or purposes allowed by the network.

\subsubsection{Infrastructure Access and Use}

Digital infrastructure ${ }^{25}$ studies has long recognised the dual role of infrastructures. On the one hand, their primary role is to ensure interconnection among heterogeneous and diverse actors that are geographically dispersed and experience localised and situated needs. On the other hand, infrastructures also require standardized and customised rules of use in order to ensure interoperability among localised and heterogeneous actors. The presence of universal rules defining and regulating the way in which the various actors accessing, and interconnecting to the network can use and operate the infrastructure itself is a necessary condition in order to guarantee the coherence, consistency and integrity of the system. The dual role of digital infrastructures unveils their dual nature, which gives rise to various paradoxes of digital infrastructures (Tilson et al., 2010; Lyytinen et al, 2018), as highlighted by the information system literature

Thus, Star and Ruleder (1996) discuss the tension between local and global concerns emerging from the duality between dispersed system access and centrally designed standards of use. Hanseth and colleagues (1996) explore the tension between interconnection of dispersed actors and the need to standardise the patterns of use of the infrastructure. Rolland and Monteiro (2002) inquire the tensions emerging between local adoption and use of infrastructures by actors operating in local contexts and the need for global and standardised control over the rules of use. Edwards et al (2007 and 2009) highlight how any infrastructure grow and expand first by incorporating heterogeneous components through interconnection, access and participation of new users, and subsequently by developing common standards regulating the nature of services and uses that can be shared and transacted by the heterogonous users. Similarly, Tilson and colleagues (2010) note that the impossibility to confine infrastructures to specific functions or predefined nodes fosters the paradoxes of change and control. In the same vain, Costantinides and Berrett (2015) highlight how infrastructures are at the same time integrators of dispersed and heterogeneous information spaces (bottom-up), but at the same time require clear design rules governing their collective and shared use (top-down). Rodon and Hanseth (2015) and Bygstad and Hanseth (2016) summarise the dual nature distinguishing between the architecture of an infrastructure shaping interconnection and its

\footnotetext{
${ }^{25}$ We will, for the purpose of the arguments in this report, assume that the concept of a "digital infrastructure" can be treated in similar terms as an "information infrastructure". While early works (for example; Gray, 1996; Hanseth \& Monteiro 1997; Kahin, 1998) used the latter, contemporary work is increasingly based on the former concept (such as: Tilson et al, 2010; Henfridsson and Bygstad, 2013).
} 
governance, which deals with the behavioural rules defining control and coordination of the activities undertaken over the infrastructure.

In sum, the aspects of access in the digital infrastructure literature characterises the inherent role of infrastructures in interconnecting heterogeneous actors over space, while the dimension of use denotes the inherent neutrality and convergence of digital infrastructures, which can be employed and allocated across a variety of uses, functionalities, services or purposes. The literature usually complements this picture by highlighting the problems and difficulties emerging from the growth and change of digital infrastructures over time along both dimensions of use and access (Bygstad and Hanseth 2016; Bowker et al 2010; Ciborra et al., 2000; Edwards et al. 2009; Hanseth and Lyytinen 2004; Monteiro et al 2013; Tilson et al., 2010). The introduction of time completes the picture by generating a three-dimensional framework for the study of digital infrastructures: over scale or space (access or interconnection), over scope or functions (use), and their evolution over time (change in both dimensions) (see also Bharadwaj et al., 2013).

\subsubsection{Platform Access and Use}

Similarly to the literature on digital infrastructures, the platform literature has also studied and analysed the platform concept along the two independent dimensions of access and use, even though it adopts a slightly different terminology and conceptual framing. In this case the interplay between access and use is usually framed in terms of the dichotomy between governance and architecture, or through the tension between value creation and value capture, which closely reflects the infrastructures' paradox of control (Ciborra et al., 2000; Tilson et al., 2010). On the one hand platform owners should open the platform to as many actors as possible, thus maximizing the adoption of the platform and the number of users able to access the platform and the number applications supported by it. Openness to interconnection (architecture) and participation maximizes value creation. On the other hand, platform owners are only able to capture some value by setting clear rules defining the way in which the platform can be used and how the various modules can interconnect and interoperate (governance) (Evans and Schmalensee, 2016).

The tension between access and use has been reframed in various ways by the platform and managerial literature. Gawer and Henderson (2007) highlight the dichotomy between control of the ecosystem architecture and free entry to maximise the ecosystem reach. In their definitions of platforms, both Baldwin and Woodward (2009) and Gawer (2009b) highlight the tension between the need to control the core and the interfaces in order to ensure stability and predictability over the way in which the platform can be used, and the need to open the platform to third parties' components and complements in order to pursue variety and variability. The same tension between access and control is also discussed by Eisenmann et al (2009) in relation to the two dimensions of openness: openness as interconnection, interoperability and compatibility with other systems, and openness in terms of the scope of exclusive functionalities and services supported by the platform. This same interpretation of the duality is also discussed in Benlian et al. (2015). Boudreau (2010 and 2012) distinguishes between two types of openness of a platform: openness deriving from granting access to it (permission to use it), and openness deriving from devolving control over how the platform should be used (definition of its functionalities). The same tension between access and interconnection and control over use is highlighted by the IS literature in Gulati et al. (2012), Ghazawneh and Henfridsson (2013), Eaton et al. (2015), and Tiwana (2015). Finally, Tiwana et al. (2010) employ the same distinction adopted by the digital infrastructure literature, distinguishing 
between architecture (interconnection) and governance (rules of use), while De Reuver et al., (2018) note that the same paradoxes of control and adaptability characterising digital infrastructures also apply to digital platforms.

In sum, the standardization and openness of interfaces ensuring the modularity and the wide adoption of the platform among a variety of actors can be applied and understood along two different dimensions. On the one hand, the platform can be open to other ecosystems, meaning that it ensures interconnection and interoperability across different ecosystem; in this case, the platform can be accessed by a multitude of users, meaning that the wide adoption of the platform is a function of the dimension of access - it is integratable (Kazan et al, 2018). Eisenmann et al (2009) and Benlian et al. (2015) define this as the "horizontal dimension" of openness (and modularity). On the other hand, the platform's modular interfaces can be open to a variety of functions or services provided by third parties' components and complements. In this case, the modularity brought about by standardised interfaces ensures an augmented set of functionalities and uses available to the platform's users - the platform is integrative (Kazan et al, 2018). The platform's adoption is in this case a function of the dimension of use (rules defining control over its functionalities and services). In the words of Eisenmann et al (2009) and Benlian et al. (2015) this is the "vertical dimension" of openness (and modularity).

\subsubsection{Platforms and Infrastructure Duality}

The dual nature of digital platforms and infrastructures retraces their dual role within a network, as commonly highlighted in the academic literature. For instance, Gawer (2014) distinguishes between the economic approach and the engineering approach to platforms. The former interprets platforms as central intermediaries providing interconnection and coordination capabilities to independent actors operating on different sides of a market place. Cusumano et al (2019) define these types of platforms as "transaction platforms". The engineering approach latter is closer to the digital approach to platforms, which interprets a platform as a core building block providing an essential functionality to a variety of other modules and components, thus acting as a common foundation for many other additional services and uses (Baldwin and Woodward, 2009; de Reuver et al., 2018; Gawer, 2009b; Tiwana et al., 2010). Platforms defined along the vertical dimension of use have recently been called "innovation platforms" by Cusumano and colleagues (2019).

The same holds for infrastructures as they fulfil both roles of interconnection tools among otherwise independent actors, and of general purpose or neutral tools for the flexible accommodation of unforeseen and diverse services or uses among actors having heterogeneous and evolving needs (Edwards et al 2009; Hanseth et al., 1996; Hanseth and Lyytinen 2004; Tilson et al 2010; Zittrain, 2008).

The dual nature and role of digital platforms and infrastructures implies that the concepts of modularity, standards and interfaces can also be intended along either dimension: as a way to enable seamless interconnection of heterogeneous modules and components along the horizontal dimension of access, and as a way to enable the implementation of heterogeneous services over a set of different physical supports. Modularity and interfaces along the vertical dimension constitute the layered stack of digital technologies (Fransman, 2010; van Schewick, 2010; Yoo et al., 2010; Zittrain, 2008). 
The dual nature of digital platforms and infrastructures retraces a well-known argument developed in various fields, from the philosophical and sociological literature on institutions (Searle, 1969) and law (Smith, to information systems to science and technology studies (STS): any artefact can be employed in a variety of uses depending on who employ (access) them. This duality is especially prominent in the case of digital artefacts (Kallinikos et al., 2013). The dual nature of digital platforms and infrastructures emerges from a loose coupling between access and use. The definition of who is able to employ the artefact is not informative of the way in which the same artefact will be used by those that are able to access it. Equally, vice versa, the definition of the way in which an artefact shall be used does not necessarily defines who will be able to do so (access it).

While the literature tends to associate the horizontal (access or interconnection) dimension of a digital network to its infrastructural nature (infrastructures interconnect), and to link the vertical dimension of use to the modular nature of a platform (Alaimo et al., forthcoming; Costantinides et al., 2018; Flew, 2019; Hanseth and Lyytinen 2010), this approach does not acknowledge the fact that both platforms and infrastructures have a dual nature and fulfil a dual role. Although our purpose in this paper is not to provide analytical criteria to distinguish the concept of platform form the one of infrastructure, we nevertheless remark that digital platforms and infrastructures both interconnect and coordinate independent actors (access), and both represent an underlying common support or functionality for the organization or transmission of data and can be devoted to a variety of uses by the part of the different interconnected actors for the provision of a variety of services and applications.

For this reason, although we do not make any claim on the matter, we suggest that the difference between digital platforms and digital infrastructures might lie in the different way in which interdependencies along the two dimensions of access and use shape the nature and behaviour of digital networks.

\subsection{A De/centralization Framework}

From the previous discussion, it emerges that any digital network can be studied along two dimensions: the dimension of access and interconnection (space) and the dimension of use - can alternatively be characterised in terms of service, purpose, or functionality. Both platforms and infrastructures can be understood and studied along either dimension. Access defines the inherent capability of both platforms and infrastructures to interconnect different actors who would otherwise be independent, and to transform independencies into interdependencies among these actors. Use defines the inherent capability of both digital platforms and infrastructures to support a variety of applications and services and to be used for a variety of functionalities and purposes. This capability of a digital artefact to be employed across a multiplicity of uses is sometime intended as neutrality, other times it is referred to as its generativity (Zittrain, 2006; 2008) or reprogrammability (Kallinikos et al., 2013; Yoo, 2013; Yoo et al., 2010 and 2012) whenever these new uses emerge expost and were not necessarily predefined or conceived ex-ante, when the network was originally deployed. In the case of generativity and reprogrammability the vertical dimension of use is inextricably linked to the temporal dimension.

Given the dual nature of both platforms and infrastructures, it is possible to understand the de/centralization of a digital network by looking at the a/symmetric interdependencies emerging 
along the two dimensions. Based on the definition of vertical and horizontal dimension, each dimension is characterised by the interdependencies between two different elements.

The horizontal dimension is characterised by a plurality of social actors willing to interact with others by accessing the physical network and interconnecting to it. The latter can be intended as the sum of the underlying physical and logical elements enabling and supporting these interactions. The two key elements of the horizontal dimension are therefore the set of actors of a social community willing to access the networked community and interact with each other, and the set of elements constituting the physical network and enabling these interactions, or "network topology". The network topology includes both the way in which the elements are spatially located, organised and physically interconnected to one another (the architectural dimension), and the rules defining the way in which the various elements can interact, interconnection is established, and the way communication can flow between them (logical dimension). The logical dimension is usually taken care by transmission and communication protocols, standards and communication interfaces. In other words, the network topology represents what has been previously defined as the technological dimension of a network, while the interactions among the various members of the social community define the social, or inter-personal, dimension of the networked system.

The vertical dimension is characterised by a plurality of services or functionalities conveyed, supported and delivered by the physical network to its various nodes through the transmission of data (and ultimately enjoyed and consumed by the various members of the interconnected community). The two key elements of the vertical dimension are therefore the set of data transmitted and supported by the physical network, and the set of rules and components of the physical network supporting and enabling the transmission of these data or, once again, the "network topology" (architectural and logical). Data, on the other hand, constitutes the symbolic dimension: as digital data is neutral, homogeneous and ontologically amorphous (Kallinikos et al., 2013; Yoo et al., 2010), their nature, valence and meaning depends on the specific symbolic framework adopted to interpret it and translated it into services, applications, and useful functionalities for those who employ and use them.

Horizontal and vertical dimensions can then be decomposed into their three core elements: 1) the social community, 2) the digital network (architectural and logical topology), and 3) the digital data. Each dimension characterises the interactions and interdependencies between a pair of elements. The horizontal dimension (access) can be understood as the locus of the dynamic interactions and interdependencies emerging between the members of the social community (SC) and the network, while the vertical dimension (use) becomes the locus of the dynamic interactions and interdependencies occurring between the network and the data exchanged and transmitted on it. These data are employed in order to provide useful service or applications that will be eventually used, consumed, and enjoyed by the various members of the social community interconnected to the network. This last linkage closes the circle of interdependencies between social community, network technology, and, data, even though it does not belong to anyone of the two dimensions.

The same framework can be better understood by decomposing the technological dimension (NT to be intended as network technology, or network topology) into its architectural component $\mathbf{A}$, and its logical component $\mathbf{L}$. The network architecture $\mathbf{A}$ represents the physical organization of the network, while the logic $\mathbf{L}$ represents the protocol rules regulating the behaviour of the network and its 
components, such as the rules defining the way in which the signal is transmitted or defining how communication and interconnection between the elements is established. In a general protocol suite, A would correspond to the physical (maybe link) layer, while $\mathbf{L}$ to the internet (network) and transport layers (see Tanenbaum and Wetherall, 2011).

In this case the vertical dimension comprises the three basic elements of the digital layered stack, the physical layer, the transmission protocol layers (TCP and IP protocols of the internet and network layers), and the data or application layer (D) (Fransman, 2010; Tanenbaum and Wetherall, 2011: van Schewick, 2010; Yoo et al., 2010), while access and interconnection along the horizontal dimension depend on both the physical architecture of the network and its logic. This is illustrated in Figure 4.1.

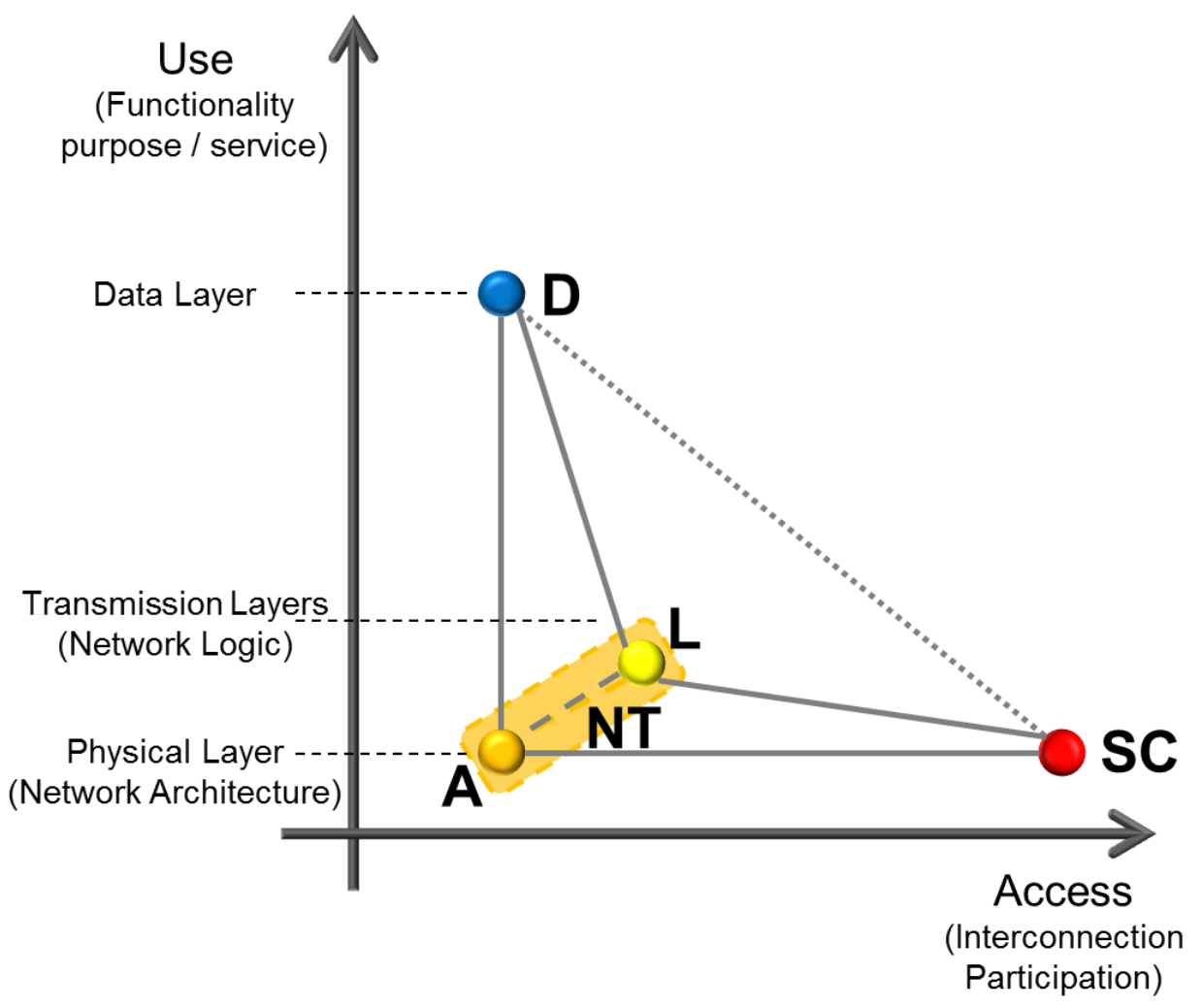

Figure 4.1: A topological overview of the conceptual model of inter-dependencies between Data (D), Network Technology (NT), and Social Community (SC) across the dimensions of Access and Use in digital networks.

These dynamic interactions and interdependencies between pairs of elements are also dynamic interactions and interdependencies between pairs of ontological and analytical dimensions. While the horizontal dimension of access links the technological dimension of the network to the social dimensions of the social community, the vertical dimension includes the interactions between the technological dimension of the network technology and the symbolic dimension of (digital) data. This reveals two different types of dimensions playing a role in the analysis of digital platforms and infrastructures. There are dimensions of first-order (fundamental dimensions) that are characterised by the interactions and interdependencies among homogeneous elements (components or actors) within a single institutional layer or ontological domain. Second-order dimensions (super-dimensions) are characterised by the dynamic interactions and interdependencies occurring between first-order 
dimensions - thus across heterogeneous institutional layers and ontological domains. This is graphically illustrated in Figure 4.2 below.

The decomposition of the two dimensions of platforms and infrastructures into its four core elements shows that digital platforms and infrastructures are composed of four fundamental dimensions, which we from now on will group into three as we consider the physical network architecture (A) and network logic (L) of the network technology as one theoretical category Network Technology (NT). The technological dimension captures the interdependencies between interacting technological components from both an architectural and logical perspective. The symbolic dimension of the data layer covers the symbolic interactions and interdependencies among different types of data and information signals. The social or interpersonal dimension is itself a sociopolitical system that includes the relationships, interactions and interdependencies between various actors and individuals. Each of the three fundamental dimensions is characterised by a certain degree of a/symmetric interdependencies among its homogeneous elements.

At the same time, the acknowledgement of the dual nature of digital platforms and infrastructures shows that these three fundamental dimensions are linked to each other and interact along the super-dimensions of access and use.

The decomposition of digital platforms and infrastructures into first-order dimensions (defining their fundamental building blocks) and second-order dimensions (defining the two axes along which these fundamental building blocks are linked) shows that interdependencies in digital networks occur at two different levels. Exactly for this reason they have a dual meaning: interdependencies among homogeneous elements within a given dimension, and interdependencies among heterogeneous dimensions along the dimensions of access and use. Consistent with the terminology adopted before, we name these first-order and second-order interdependencies. Both first- and second-order dimensions are systems of interacting elements; they are just defined at two different analytical levels of aggregation.

For this reason, while each of the basic building blocks of any digital network can be defined as a first-order system, a digital platform or a digital infrastructure can be regarded as a second-order system, or a system of systems. The fundamental characteristic of a secondary system is that interactions and interdependencies occur across institutional layers and have to be defined across ontological domains. For this reason, second-order systems are also multi-dimensional. For instance, the definition of infrastructures as socio-technical systems (Edwards et al, 2007; Henfridsson and Bygstad 2013; Karasti et al, 2010; Star and Ruhleder, 1996) makes the multidimensional nature of infrastructures explicit deriving from the interactions between the social and the technical dimensions. We illustrate this in Figure 4.3. 


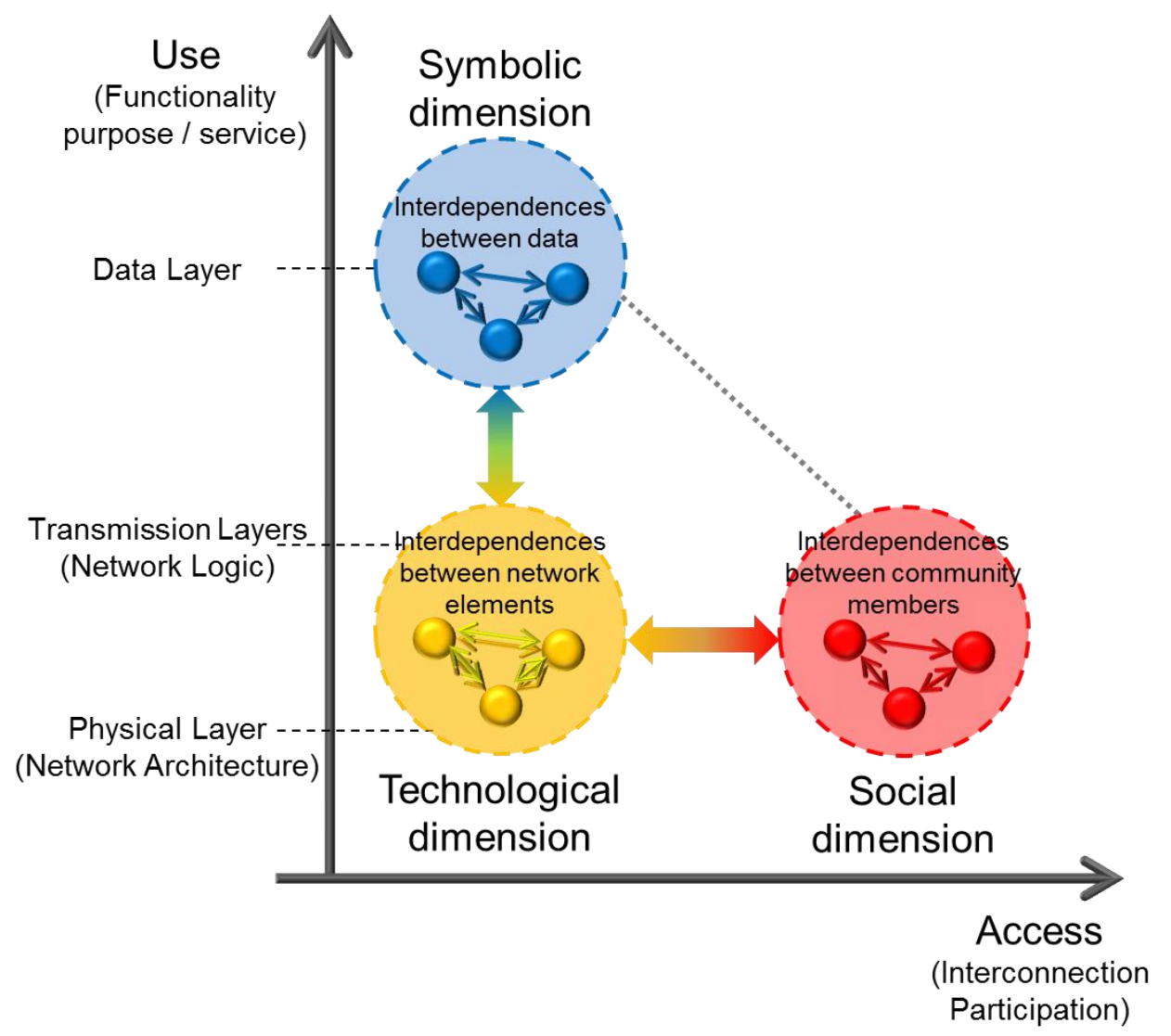

Figure 4.2: Topological illustration of the dual nature of digital platforms and infrastructures along the three fundamental dimensions along the super-dimensions of access and use.

Accordingly, it is possible to define de/centralization at two different analytical levels: at the firstorder level and at the second-order level. The latter builds on the former, but plays a very different role in understanding de/centralization in digital networks.

De/centralization at the level of the single dimension (first order) emerges as the result of a/symmetric interdependencies among the homogeneous components belonging to that dimension. The analysis of de/centralization at this level considers each dimension (the analytical and ontological locus) separately and independently from the others - as an insulated and autonomous system. For this reason, the nature of the interactions and interdependencies among the elements within each dimension are taken as given. The interactions are exogenous characteristics of the single dimension that are merely verified and described. In order to explain de/centralization at the systemic level (second order), it is fundamental to understand how first-order interdependencies among elements in one dimension both affect and are affected by the first-order interdependencies among elements in other first-order dimensions.

De/centralization in second-order systems emerges as interdependencies of interdependencies. These interdependencies of second-order characterise the way in which interdependencies of first order within one ontological domain are interdependent on the interdependencies of first order among the components within another ontological dimension. 


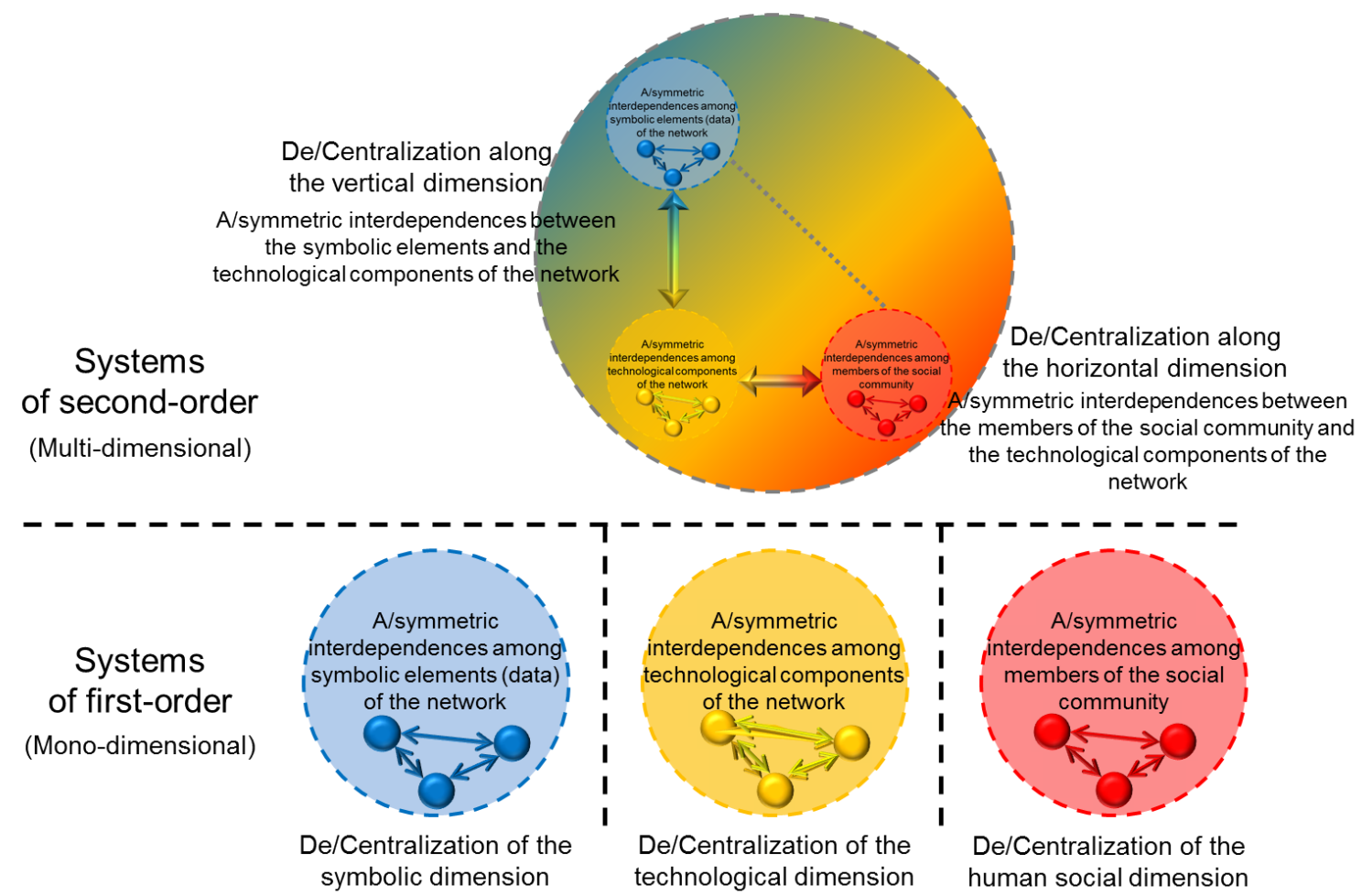

Figure 4.3: An illustration of the distinction between first order and second order systems.

Differently from the case of first-order systems, the concept of decentralization in the case of digital networks does not characterise the particular state of a dimension, nor a mere sum of states of the different independent dimensions, but the peculiar way in which various dimensions of a system interact among themselves and influence each other's states and behaviours. While the study of first-order interdependencies provides a snapshot of the relationship of dependence for each dimension, the study of dependencies across dimensions provides a perspective on the temporal and logical causality of the interdependencies by showing how the degree of a/symmetric interdependencies in one dimension depends on the extent of a/symmetric interdependencies in other dimensions, and vice versa. Stated differently, second-order levels define the way in which relationships of dependence among elements within each first-order dimension are in turn shaped by the relationships of dependence across first-order dimensions.

For this reason, an analysis of second-level interdependencies is the only way to endogenously derive the degree of de/centralization in each first-order dimension as a function of the degree of de/centralization in the other first-order dimensions it interacts with. It is, precisely, an analysis of de/centralization of second order that recognises the interdependencies are themselves the outcome of higher-level interdependencies.

We argue that when dealing with multidimensional systems such as digital platforms and infrastructures, interactions at all levels must be considered. Any digital network (no matter whether it is intended as a platform or an infrastructure) is a multi-dimensional system (a system of systems) composed of three fundamental dimensions of first order (defining the four core elements of data (D), social community (SC), and the network architecture (A), and logical architecture (L) from Figure 4.1) dynamically interacting along the two dimensions of access and use. As digital platforms and 
digital infrastructures are multidimensional concepts, the concept of de/centralization also becomes multidimensional, as also remarked by other authors (for example; Schneider, 2003; 2019).

As a result, once the single elements are characterised, the nature of any digital network (including its degree of de/centralization) can only be understood by looking at the nature of the interdependencies between these elements across disciplinary fields and ontological dimensions. Treating the degree of de/centralization of a digital network as a mere sum of independent dimensions or elements might not generate the appropriate understanding of the degree of de/centralization in digital networks.

\subsection{De/centralization in Digital Networks}

\subsubsection{First-Order Interdependencies}

The extent of de/centralization of each fundamental dimension defines the extent to which the nature or state of one element depends on the nature or state of others. At this level, de/centralization for each dimension can be defined as the extent to which the state, nature or role of any element of that primary dimension is dependent on the states, natures or role of other elements in that same dimension for given interdependencies occurring in the other two dimensions. In other words, at the primary level the degree of de/centralization in each dimension is defined as a feature of the dimension itself, independently from what is happening in the other dimensions.

\section{a. First order dimension 1: Technological de/centralization}

A digital network might be more or less centralized from a technological point of view if the ability of a network element to fulfil its role within the network and to perform its expected functions depend, in a more or less a/symmetric fashion, on other elements performing their functions and fulfilling their roles. The study of interdependencies characterising the network topology adopts a technological approach to de/centralization whenever the interdependencies among the elements of the network are understood and evaluated adopting a normative framework that relies on technological interpretative categories.

De/centralization from an architectural point of view is defined by looking at the nature of interdependencies among elements that emerge as a result of the way in which components are spatially arranged and organized. Asymmetries are present whenever the ability of a component to perform its functions depends on its location within the network and on its interconnection with other specific components. Whenever the ability of a network component to perform its functions is independent from the way in which the network is designed, or whenever there is mutual interdependence among the various components, interdependencies are symmetric from an architectural point of view. As already remarked, the architectural approach to de/centralization emerged in computer science (Baran, 1964), and then spread to a multiplicity of other fields, especially the one developing network analysis. The presence of a hub-and-spoke architecture based on a central intermediary is a straightforward example of architectural asymmetries as the ability of any peripheral component to fulfil its role within the network depends on the state or the behaviour of the hub. A distributed network where all components can communicate in equal measure with every other component (full redundancy) characterises a perfectly symmetric situation.

On the other hand, from a logical perspective, technological centralization occur whenever there are asymmetries in the way in which communication is transmitted or, in other words, in the respective 
roles that different nodes have in running the protocol or routing the information. More generally, any decentralized architecture can become logically centralized whenever there are asymmetric roles and capabilities among the network elements. A client-server design is a centralized solution from a logical point of view as different nodes perform different roles within the network. Peer-topeer networks are logically decentralized as they require the equality of all nodes with respect to the way in which the content can be transmitted, but they do not necessarily need to be architecturally decentralized (Agre, 2003).

\section{b. $\quad$ First order dimension 2: symbolic de/centralization}

Data belongs to the symbolic sphere. The study of the asymmetries emerging between data might follow a variety of normative and interpretative approaches (social, economic, legal, political) depending on the specific perspective adopted to evaluate and treat these data. The data can be endowed with a social valence (they might be evaluated based on their contextual utility and benefits deriving from the use or consumption of the service generated), economic value (data evaluated based on their monetary value in exchange), legal valence (data having value as property, as in the case of privacy), or even political value (data used to influence opinions and shape public discourse).

De/centralization in the symbolic dimension can be understood by looking at their symbolic interdependencies, which we define as the degree in which the meaning, value, and nature acquired by a digital data within a broader communication conveyed by the network depends on the meaning, value, and nature acquired by other data, and vice versa. Data are interdependent whenever different data have different value, nature, and meaning - when they have to be organised and managed in an ordered and coherent way based on their different contribution to the meaning and value of the final outcome to be provided (service or application). This generates rankings, priorities, or discrimination among different data. This interdependence can be symmetric whenever changing the symbolic meaning and nature of any data corrupts the overall meaning of the final outcome, thus affecting the meaning of all other data involved and vice versa. Asymmetries among data emerge whenever the knowledge of the symbolic meaning or value of one data is necessary in order to make sense of other data, but the former is not affected by a corruption of, or a change in, the meanings of the latter. In this case the meaning and value acquired by some data depend on the nature of some specific data. In this situation the independence between data emerges whenever the symbolic meaning acquired by a data becomes irrelevant for another one - the meaning of the latter does not depend on the value of the former and vice versa.

Asymmetric interdependencies among data might generate centralization as the meaning of some data can only be known, interpreted, or accessed by few actors. Whenever communications have to be encrypted or codified, the symbolic dimension of data is certainly characterised by strong interdependencies as it is necessary to know the value or meaning of some data to decrypt the whole message and retrieve the meanings and values of all other data. Asymmetries in these interdependencies among data signals generate centralization at the symbolic level. Problems linked to the private treatment of data, profiling, and identification of the underlying meanings and values of certain digital data generated online (usually addressed through encryption) are all linked to the degree of de/centralization of the symbolic dimension. 
More generally, whenever the symbolic dimension manifests interdependencies, there is a need to agree on some shared rules of communication defining meanings, nature, and value of data (how data should be interpreted, treated and understood). De/centralization in this case emerges from the emergence of some form of control in the way in which data is organised and the message is transmitted. This is evident when data can assume different nature: different patterns of interdependencies are generated by different types of data and this might require different rules for the organization of the transmission of this data. A straightforward example might be the transition from the "best effort" approach in data transmission of the original internet when only "downloadable" content had to be transmitted, to the transmission of media content or financial data, where a certain degree of organization of the message becomes fundamental. In the first case, whenever all data can be treated as pure content to be downloaded, such as in the case of a web page on the internet or an email, digital data can be treated as homogeneous and substantially symbolically independent from each other. In the second case, whenever data represents financial rights or economic value, their degree of interdependence is definitely higher as the value and meaning of one might depend on the value assumed by another one. When the nature and value of a data are strongly interdependent on others, jitter, latency, and errors in the order or transmission might all be fundamental network problems corrupting the meaning and value of the overall transmission. Temporal interdependence in this case plays a fundamental role, while it does not in the case of pure content.

\section{c. First order dimension 3: social de/centralization}

Finally, a digital network might be more or less centralized depending on the nature of the interdependencies characterising its social dimension. Similar to the definition given for the other two dimensions, de/centralization in this case can be defined as the extent to which the role played by a member of the community when accessing or using the network depends on the decisions or actions of other actors of the social community and vice versa.

Similar to the case of the symbolic dimension, the asymmetries emerging among the members of a social community can be analysed and understood adopting a variety of perspectives, depending on the nature of the factors that generate inequalities and asymmetries among the members accessing and using the network. Inequalities might be purely geographic whenever the ability of some member to access and use the network depends on their location vis-à-vis others. Inequalities might have a legal origin due to different rights to access or use the network. Alternatively, asymmetries can have a socio-economic or cultural nature as different actors might have different needs, capabilities or reasons for using the network. In this case, inequalities among members derive from the fact that some might derive greater benefits than others from accessing and using it, or from the fact that some members depend on others in order to enjoy the services and benefits provided by the network. Finally, asymmetries in accessing the network might be interpreted through a political lens, as in the case of concepts as the ones of universal service, public service, essential facility, privacy, and so on. In this case, interdependencies among members have a legal origin. Table 4.1 summarizes de/centralization in interdependencies across the three dimensions. 


\begin{tabular}{|l|l|l|l|}
\hline $\begin{array}{l}\text { First-order } \\
\text { dimension }\end{array}$ & $\begin{array}{l}\text { Unit of analysis } \\
\text { (element) }\end{array}$ & $\begin{array}{l}\text { Interpretative approach } \\
\text { adopted }\end{array}$ & Definition of de/centralization \\
\hline \hline Technology & $\begin{array}{l}\text { Network } \\
\text { Technology (NT) }\end{array}$ & Architectural (A) & $\begin{array}{l}\text { Technological interdependencies: } \\
\text { The extent to which the ability of a network } \\
\text { element to fulfil its role within the network and to } \\
\text { perform its expected functions depend, in a more } \\
\text { or less a/symmetric fashion, on other elements } \\
\text { performing their functions and fulfilling their } \\
\text { roles, and vice versa. }\end{array}$ \\
\cline { 2 - 4 } & Digital Data (D) & $\begin{array}{l}\text { Political / economic / legal / } \\
\text { cultural } \\
\text { (depending on the normative } \\
\text { framework adopted to } \\
\text { conceptualise the nature of } \\
\text { data as fundamental } \\
\text { informational elements of a } \\
\text { symbolic system) }\end{array}$ & $\begin{array}{l}\text { Symbolic interdependencies: } \\
\text { The extent to which the meaning, value and } \\
\text { nature acquired by a digital data within a broader } \\
\text { symbolic information transmission conveyed by } \\
\text { the network depends on the meaning, value and } \\
\text { nature acquired by other data, and vice versa. }\end{array}$ \\
\hline Social & $\begin{array}{l}\text { Members of the } \\
\text { social community } \\
\text { (SC) }\end{array}$ & $\begin{array}{l}\text { Political / economic / legal / } \\
\text { cultural / spatial } \\
\text { (depending on the normative } \\
\text { framework adopted to } \\
\text { conceptualise the nature of } \\
\text { actors as fundamental } \\
\text { members of a social } \\
\text { community) }\end{array}$ & $\begin{array}{l}\text { Social interdependencies: } \\
\text { The extent to which the role played by a member } \\
\text { of the community when accessing or using the } \\
\text { network depends, in a more or less a/symmetric } \\
\text { fashion, on the decisions or actions of other } \\
\text { actors of the social community and vice versa. }\end{array}$ \\
\hline
\end{tabular}

Table 4.1: A summery of de-centralization across the network technology, symbolic, and social dimensions.

\subsubsection{Access and Use Interdependencies}

The study of first-order dimension interdependencies is necessary but not sufficient to understand de/centralization of digital networks as such analysis provides a snapshot of the current states of each dimension, independently from each other. As a result, inter-dimensional dependencies are ruled out or ignored. This mono-dimensional approach to de/centralization is the one adopted by the overwhelming majority of the literature in the study of de/centralization in digital networks (and blockchain): de/centralization is treated as a sum of the characteristics of a set of independent dimensions. For this reason, this also implies a static, or exogenous, approach to institutions.

The understanding of first-order interdependencies is, however, a necessary precondition in order to have a full characterization of de/centralization in digital networks. It is first necessary to understand what defines de/centralization at the level of the single dimensions in order to be able to define de/centralization at the second-order level. This can be defined as follows: the extent to which the degree of asymmetric interdependencies in one first-order dimension is interdependent (in a more or less symmetric fashion) on the degree of asymmetric interdependencies in other first-order dimensions, and vice versa. This is a definition of interdependencies of interdependencies (secondorder interdependencies) that defines de/centralization of a system as the ability of a fundamental dimension to influence and affect other dimensions' characteristics, and vice versa. 


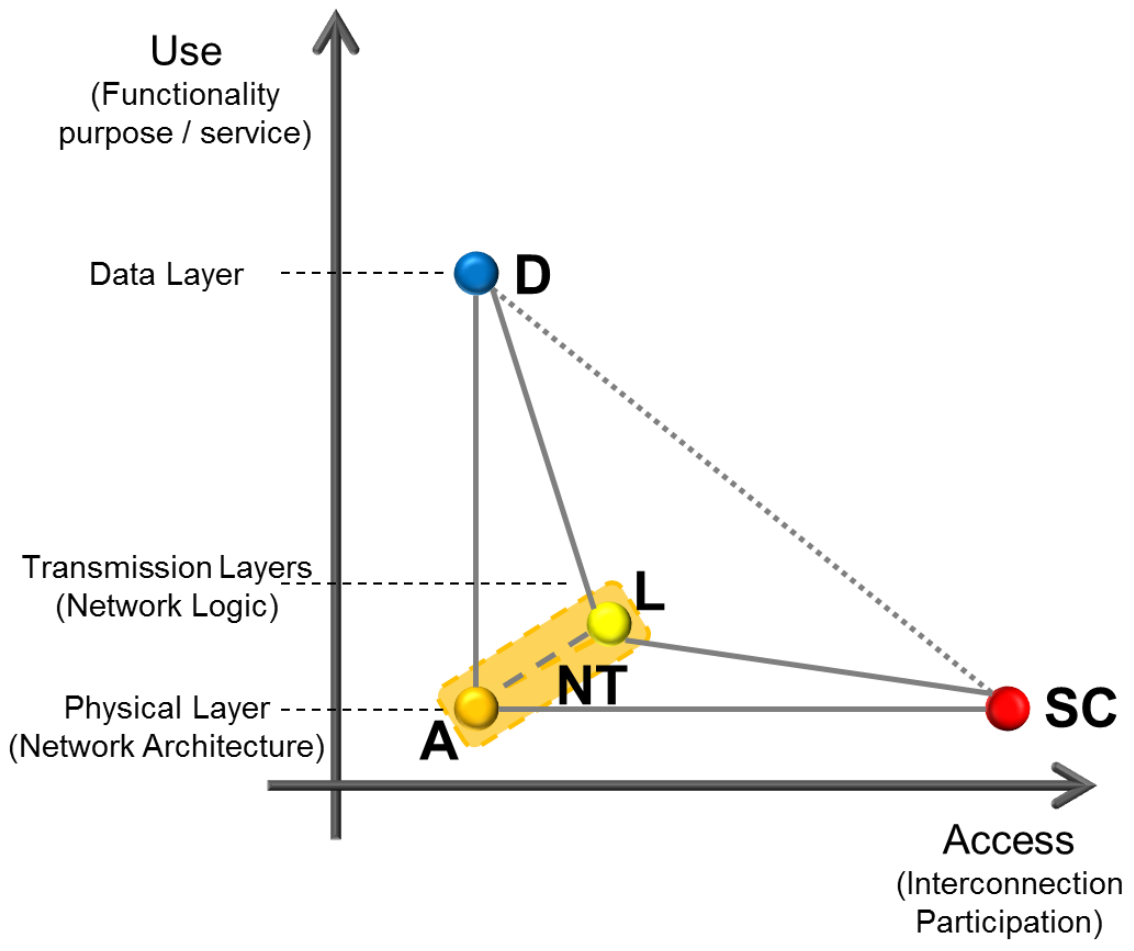

Figure 4.4 (identical to Figure 4.1 and included for easy reference): A topological overview of the conceptual model of inter-dependencies between Data (D), Network Technology (NT), and Social Community (SC) across the dimensions of Access and Use in digital networks.

A fundamental difference between first-order interdependencies and second-order interdependencies derives from their different temporal nature: first-order de/centralization characterise a-temporal (static) interdependencies in force in a certain dimension, while secondorder de/centralization reveals the causal (temporal) relationships behind first-order interdependencies. Symmetric interdependencies (mutual dependence) between two primary dimensions reveal a situation of endogenous (reciprocal) change between the two. Asymmetric interdependencies (relationships of dependence) denote a situation where asymmetries in one dimension are causally shaping and driving asymmetries in other dimensions - independence characterises a situation of no causality. For this reason, the study of how different dimensions of a complex system endogenously interact and influence each other's states and characteristics is also a theory of endogenous institutional change, understood as an endogenous and dynamic interplay between different institutional planes (Abdelnour et al., 2017).

The intuition here is that a/symmetries in one dimension do not merely affect the way in which the elements of that specific dimension interact, but they also have "spillover effects" in other dimensions. They are also affecting interdependencies among the elements of these other dimensions. For this reason, a mere analysis of de/centralization within single dimensions might be a partial one, providing a distorted understanding of the extent of de/centralization of a digital network.

Based on our definition of de/centralization as a/symmetric interdependencies, it is possible to analyse the role played by both the platform and the infrastructural nature of digital networks by looking at the a/symmetries emerging along both dimensions of access and use. 
a. Socio-Technical interdependencies in digital networks: de/centralization along the horizontal dimension of access

The horizontal dimension (see Figure 4.4) can be defined as the set of interactions between the members of the social community (SC) and the technological dimension of the network broadly defined (NT). The interdependencies occurring along the NT-SC linkage capture the way in which the nature and characteristics of a social community shape the nature and characteristics of the network topology (SC $\rightarrow \mathbf{N T}$ ) and, vice versa, how the nature and characteristics of the network in turn affect and shape the nature and characteristics of the social community (NT $\rightarrow$ SC). This second-order interdependence captures the fact that the nature of interdependencies among the actors of the social community might depend on the specific interdependencies in force between the elements of the network and, vice versa, the latter might be the result of the former.

Interdependencies between the social and the technological dimension can be symmetric, asymmetric or inexistent, depending on the causality of interaction. On the one hand, these interdependencies emerge because the way in which the social community is spatially organized and geographically dispersed affects, and is affected by, the physical organization and location of the network's elements ( $\mathbf{S C} \Leftrightarrow \mathbf{A}$ ). On the other hand, interdependencies arise because the way in which signals and communication is transmitted online affects, and is affected by, the patterns of communication and interaction among the actors of the social community offline (SC $\Leftrightarrow \mathbf{L}$ ). While the social community-physical layer (SC $\Leftrightarrow \mathbf{A})$ relationship mainly captures spatial (static) interdependencies in access, the social community-logical layer (SC $\Leftrightarrow$ L) relationship mainly captures dynamic interdependencies between the way in which interactions are organised and conducted offline (among the members of the social community) and the way in which interactions are organised and conducted online (among the nodes and components of the physical network). For blockchain, the dynamic interdependencies emerging between the logical dimension of network topology (P) and the social dimension of the social community $(\mathbf{S C})$ is usually framed as the interplay between "code as law" ( $\mathbf{L} \rightarrow \mathbf{S C}$ ) and "code of law" (SC $\rightarrow \mathbf{L}$ ) or, similarly, between "code is law" and "law is code" (De Filippi and Hassan, 2018; Yeung, 2019).

Centralization along the horizontal dimension emerges whenever these interdependencies between the social community and the network topology are asymmetric. Asymmetries in this case emerge whenever the interdependencies within one dimension is causally dependent (determined) by the specific nature of interdependencies in force in the other dimension (and vice versa). These asymmetries can of course be of different nature, have different origins and come in different forms along the architectural, logical, political dimension. The next section will engage in an in-depth analysis of how de/centralization in blockchain arrangements can be characterized in terms of these dimensions.

Second-order centralization along the horizontal dimension can be architectural or structural $(\mathbf{A} \rightarrow$ SC). In this respect, centralization emerges whenever the dependence of a member of the social community on other members in order to access, interconnect and use the network depends on the specific architectural dependencies shaping the spatial organization of the elements of the network. This is the typical case of path dependency of a digital infrastructure (Arthur, 1989), where the ability of a member to access, interact or interconnect to the given network depends on the preexisting peculiar relationship between the nodes of the network. Alternatively, the causality can work the other way around, whenever asymmetries in the social dimension generate asymmetries in 
the architecture of the network (SC $\rightarrow \mathbf{A}$ ): centralization along the horizontal dimension emerges whenever the chosen architectural configuration of the network depends on the specific way in which the members of the social community can interact. This is the case of a local network where a specific configuration or characteristic of the network becomes preferred to others depending on where the actor willing to access and use the network is located.

The same considerations hold for the logical dimension of the network. Asymmetries emerging because the different social roles, status, or identities among the various actors of the social community can determine the protocol rules defining the way in which the various elements of the network can behave, interact, and interconnect (SC $\rightarrow \mathbf{L}$ ). In this case, a given socio-political context shapes the way in which a network is designed: communication rules online might reflect the specific patterns of interactions offline. Alternatively, it is possible to conceive a situation where the social status or roles of the members of the social community is determined by the specific logic of the network ( $\mathbf{L} \rightarrow \mathbf{S C}$ ). In this case asymmetries emerge among the members of the social community because a certain network topology favours a certain kind of actor to the detriment of other kinds of actors. This is also a form of technological path dependency: asymmetric interdependencies among network's components shape the interdependencies among the actors of the social community.

In all cases, centralizing tendencies emerge because asymmetries and dependencies among elements in one dimension foster and generate asymmetries and dependencies among elements in the other dimension. Centralization in this case denotes univocal causality from one dimension to the other. On the other hand, decentralization along the horizontal dimension denotes a situation where there is no univocal causality form one dimension to the other.

In the case of symmetric interdependencies, decentralization along the horizontal dimension denotes a situation where the specific technological solution emerges as the preferred choice of the members of the social community, but does not necessarily reflect or retrace the asymmetries characterising the social community. This misalignment between online and offline interdependencies enables new interactions and new social interdependencies among the actors of the social community. The latter might, in turn, decide to change the network topology. Differently form the asymmetric case, the specific network topology in this case does not univocally reflect the specific asymmetries of the social community, and interdependencies work both ways: the specific network topology can now, in turn, influence future community arrangements.

As a result, while the network topology depends on the specific dynamics of the social dimension, the latter, in turn, also depends on the former in a self-reinforcing cycle. As the asymmetries in one dimension are not necessarily consistent with the asymmetries in the other dimension, the interdependencies are mutual, and the influence works both way generating an endogenous dynamic cycle, which really is the essence of symmetry in interdependencies between two elements (SC $\Leftrightarrow \mathbf{N T}$ ) along the horizontal dimension. Symmetric interdependencies between the social dimension and the technological dimension can be understood as an endogenous coevolution between the social community and the network topology.

This dynamic interaction where the nature of one dimension endogenously affect the nature of the other and vice versa can also be intended as a process of institutional change (Abdelnour et al., 2017). A simple example of endogenous institutional change in the case of symmetric 
interdependencies between the social and the technological dimension is the one of technological innovation, whenever the process guiding the adoption of new technological solutions is neither codified nor properly regulated by the current social structure or political process in force in a social community. In this case, the solution adopted in terms of network topology might still reflect particular interests of the members of the social community, but is also misaligned with the current rules regulating the organization of the social community, thus generating new opportunities for dynamic change. The definition of standards and new regulatory rules generates new social interdependencies, but also requires adaptation and adjustments in the network technology.

Finally, the case of independence is simply the one developed earlier with respect to first-order de/centralization. In this case decentralization along the horizontal dimension denotes a situation where the way in which the elements of the network are organised (from an architectural (A) and logical (L) standpoint) is irrelevant for the way in which social interactions are arranged among its members. This means that the specific technological solution has no effect on the way in which these members interact and arrange their social relationships offline, and vice versa. This horizontal independence might be understood in two specular ways depending on whether the focus is on the independence of the social community from the technological solution or the other way around. It can be viewed as a case of technological neutrality (the topology of the digital network is neutral in the sense that it does not affect, or have any impact on, the specific socio-political context) or as a case of technological determinism (there is an objective technological solution that is unaffected by the specific socio-political context). In either case, second-order decentralization along the horizontal dimension defines a condition where the technological solution adopted is unaffected by the specific way in which the various members of a social community interact or, vice versa, a condition where the specific characteristics of the social community are completely unaffected by the way in which the various elements of the digital network are organised and designed.

As this decentralization occurs across elements (it defines second-order decentralization), this does not imply that there are no first-order asymmetric interdependencies among the members of the social community or among the various components of the digital network. It is possible to define independent first-order asymmetric interdependencies. In this case, we define independence to underline the situation where the nature of the interdependencies characterizing first-order elements is uninformative to define decentralization along the second-order dimension as one is not affected by the other and vice versa: second-order decentralization becomes independent from the nature of the interdependencies within first-order elements.

Although it is not clear whether it is possible to conceive a situation where technologies are politically neutral, or one where social arrangement operate in a technological vacuum, the conditions characterising second-order independencies are precisely the one usually (implicitly) assumed by the literature in the study of digital networks. A mono-dimensional approach to decentralization that only focusses on a/symmetries of first order can only be justified under the assumption that inter-dependencies of second-order across ontological dimensions are inexistent (or negligible at least). 
b. Techno-symbolic interdependencies in digital networks: de/centralization along the vertical dimension of use

The vertical dimension (see Figure 4.4) is defined by the interactions between the network (NT) and the data transacted over it (D). In this case, the interdependencies occurring between NT and D capture the way in which the nature and characteristics of the network shape the nature and characteristics of the data supported and transmitted by it (NT $\rightarrow$ D) and, vice versa, how the nature and characteristics of the data transmitted shape and affect the nature and characteristics of the network in turn ( $D \rightarrow N T$ ). A specific network topology can be chosen in function of the specific nature of data to be transmitted and in function of the type of service or functionality to be provided or, vice versa, the communication and transmission of data can change and modify its symbolic meaning in function of the specific network topology in force. This second case is especially true in the case of digital data, given its amorphous and neutral ontology (Kallinikos et al., 2013; Yoo et al., 2012).

Even in this case two sub-types of interdependencies can be identified. Interdependencies between NT and $\mathbf{D}$ might emerge because the meaning of data depends on the geographical dispersion and distribution of the various data and this might affect, and is affected by, the physical organization, arrangement and location of the network's elements (A $\Leftrightarrow \mathbf{D})$. This interdependence captures the way in which the asymmetries in the way in which the network is spatially organised and structured affect, and are affected by, the symbolic meaning of data distributed and dispersed offline. In this case asymmetries in the dispersion of relevant information might generate symmetries in the way in which the network's elements are arranged and located. The opposite can also be true: the specific asymmetries in the architectural topology of the network might affect the way in which data are collected and generated, and therefore the overall meaning and significance of the communication and information transmitted. The case of symmetric interdependencies defines a situation where the symbolic nature of data and the architectural organization of the network's elements dynamically and endogenously coevolve.

At the same time, interdependencies along the L-D link emerge as the meaning, value and nature of different types of data affect, and are affected by, the way in which these data are organized, combined and transmitted online among the various nodes of the network. This is the case where the symbolic meaning of data depends on the specific protocol rules adopted ( $L \Leftrightarrow \mathbf{D}$ ). This specific interdependence captures many aspects associated to the neutrality and convergence of digital networks, due to the fact that the type of transmission protocol can depend, and be dependent on, the symbolic nature of the various data transacted.

The way in which a certain digital network can support different uses, services, or functionality and can allow for their implementation and performance can also be characterised by higher or lower degrees of asymmetry. Asymmetries in this second case arise whenever the nature of the service or functionality that can be supported or provided is more or less dependent on the specific nature or characteristics of the underlying physical support network and vice versa. If the nature and characteristics of the underlying network favours one use over another one (the nature of the latter depends on the nature of the former) or, vice versa, whenever the nature and characteristics of the underlying physical network depends on the nature of a specific chosen use, the network manifests asymmetries over the dimension of use. Even in this case, asymmetries can emerge for different reasons and can have different origins: architectural, logical, economic, and so forth. 
For instance, a particular configuration of a network (originally devised for the transmission of a particular service or set of services) might become outdated or impractical whenever a new, different, service is supported by the network. In this case, the specific peculiarities of the different uses create symmetries among different network configurations, meaning that the provision of a certain service depends on a specific network configuration and vice versa. This is the case of the reconfiguration of the open internet from the peer-to-peer transmission of data and information to the transmission of digital value through the introduction of centralized intermediaries based on a client-service configuration. Alternatively, asymmetries might emerge in the way in which different types of services are treated and supported by a given network's configuration. The introduction of deep packet inspection and the breach of the neutrality principle based on the discrimination among different services is an example of emerging asymmetries among uses for a given network configuration and characteristics. Even in this case, decentralization along the vertical dimension denotes either a situation of mutual coevolution between the technological and the symbolic dimension, or a situation of independence between the two. The latter is particularly interesting as complete independence between data and the underlying network is exemplified by the neutrality of the original internet, where the Internet network protocol (the IP protocol) could ensure a complete decoupling between the technological layer and the data layer (Zittrain, 2008).

\section{c. Second-order interdependencies and de/centralization of digital networks: summing up}

The goal of this paper is to theoretically investigate the issue of centralization and decentralization in digital platforms and -infrastructures. The purpose is neither to theoretically define platforms and infrastructures, nor to provide a comprehensive framework distinguishing digital platforms from digital infrastructures. However, what emerges from this discussion is that centralization and decentralization are not peculiar and exclusive characteristic of either platforms or infrastructures. The dual nature of platforms and infrastructures implies that both platforms and infrastructures can be at the same time a source of de/centralization as both centralization and decentralization can simultaneously emerge along both dimensions of access and use. We can summarise the present discussion in four categories of cases depending on whether asymmetries emerge: 1) along the vertical dimension (asymmetries in use); 2) the horizontal dimension (asymmetries in access and interconnection); 3) along both dimensions at the same time; or 4) along neither. 


\section{Blockchain as a Digital Network}

The aim of this section is to apply the theoretical framing from the previous section to the case of blockchain through relating the extant blockchain literature to our conceptualization of de/centralization in digital networks, and discussing how the literature has largely failed to address the issue of de/centralization of blockchain arrangements comprehensively.

\subsection{Blockchain Dimensions in the Literature}

Blockchain has been defined in various ways by the current literature. Three main approaches can be identified, in line with the three fundamental dimensions of a digital network discussed in the previous section. One common approach adopted by the literature is to define blockchain in function of its main technological features and characteristics, such as, for example, these five aspects: 1) distributed; 2) transparent; 3) anonymous - or in most cases more precisely, pseudonymous; 4) tamper-resistant; and 5) peer-to-peer (p2p) nature. Thus, Rodrigues (2018) adopts the first four concepts to describe the blockchain, while Reyes (2018) defines blockchains as distributed ledger technologies characterised by a distributed architecture with tamper-resistant and transparent records. Wright and De Filippi (2015) identify four key features of the blockchain: 1) the $p 2 p$ architecture; 2) the decentralized storage of records; 3) the reliance on cryptographic algorithms; and 4) the operation of a decentralized consensus mechanism (see also De Filippi and Wright, 2018: chapter 2). Davidson et al. (2018) identify two key characteristics of the blockchain: p2p connections and cryptography. These characteristics, the authors argue, enable trust-less consensus in order to achieve decentralized consensus. Many other examples can be found in the literature, which usually represent a variable combination of the five aforementioned concepts.

The shortcoming of many of these works is that they fail to distinguish the necessary technological conditions that univocally characterize a blockchain network from other superfluous or accessory ones. As a result, very often means are confused with ends. The transparent and tamper-resistant characteristics of the blockchains are means to achieve an end (the distributed time stamp among all nodes) rather than goals in themselves, while anonymity (or pseudonymity) certainly is an "end" of the system. It is important to distinguish a necessary property of a blockchain technology from the variable and contingent solution adopted in order to guarantee that technological property.

Other works define blockchain mainly in function its institutional properties, by looking at how blockchain can coordinate different social actors, rather than its technological characteristics (Davidson et al., 2018). These works mainly focus on either the nature of the consensus protocol, or on the nature of the blockchain's governance mechanisms. Both strands define blockchain in function of the characteristics of its decision-making process and of the way in which the interactions among the various actors are regulated. While the former mainly look at the formation of the decision-making process among nodes on-chain, ${ }^{26}$ the latter defines blockchain in function of the decision-making process occurring among the members of the social community. The concept of "decentralization" is used to characterise the on-chain consensus protocol among the network's

\footnotetext{
${ }^{26}$ The distinction between on-chain and off-chain basically denotes what data is stored and managed directly through the combination of data and network technology along the vertical dimension in Figure 4.4, while offchain denotes activities beyond this arrangement, which are organized within a social context, or via sociotechnical arrangements, such as on-line discussion forums, but not directly embedded within the blockchain more on this later in the document.
} 
nodes (De Filippi, 2016; Kwon et al., 2019; Reyes 2016; Tasca et al., 2017; Xu et al 2017; Wright and De Filippi 2015), the off-chain decision-making process among the members of the social community (Arrunada and Garicano 2018; Azouvi et al, 2018; Dupont, 2018), or both (Abramowicz, 2016; DeFilippi and Loveluck 2016; Gervais et al., 2014; Hsieh et al., 2018; Walsh et al 2016).

When blockchain is defined in function of its "decentralized" consensus mechanism, other problems emerge, as already remarked above. The definition of blockchain in function of the term "decentralization" is here analytically unverifiable and logically unsustainable. Also, decentralization is usually conflated and confused with distribution, even though the two terms have two completely distinct meanings characterizing two different aspects of digital networks (Rauchs et al., 2018: 44-45; Troncoso et al., 2017). Moreover, the literature often fails to recognize the importance of the distinction between on-chain and off-chain consensus and coordination. This confusion is particularly evident in the numerous debates on the utility of blockchain that fail to distinguish between a productivity approach and a coordination approach to blockchain (Davidson et al., 2018). There is still a fundamental confusion in the literature between the adoption of blockchain as: 1) A productive technology used by firms as a strategic resource to increase the efficiency and productivity of the existing business practices and the speed and reliability of the settlement of inter-firm transactions; and 2) blockchain as a socio-institutional technology, which enables new ways in which unknown and independent actors can cooperate in the mutual provision and consumption of services. Efficient "decentralized" coordination on-chain does not necessarily imply decentralized coordination off-chain.

A third strand of works characterises blockchain by mainly focusing on the characteristics of the data transacted and exchanged over the blockchain network and on the nature of the incentives generated by the acquisition or access to this data. The works belonging to this third interpretative strand highlight the fact that the blockchain should be intended as an "internet of value" (Kewell et al., 2017; Swan, 2015; Tapscott and Tapscott, 2017). In this case, the feature that mainly distinguishes blockchain from other digital networks is the fact that blockchain allows for digital scarcity and therefore for the transaction of economic value and for the enforcement of economic incentives (Miscione et al., 2018).

Many of these works do not adopt a mono-dimensional understanding of blockchain, but adopt a multiplicity of perspectives, and define blockchain in function of a combination of different characteristics (Abramowicz, 2016; Hsieh et al., 2018; Pereira et al., 2019; Reyes at al 2017; Swan and De Filippi, 2017; Ziolkowski et al., 2018b). What is missing is a general understanding of how these various dimensions (and approaches) interact, how they should be conceptually linked and what their respective roles are within a broader coherent picture. On the one hand, the various aspects of a blockchain are often conflated. For example, the consensus protocol conflated with the technological characteristics of the underlying network, or the properties of the coordination mechanisms on-chain with the ones of-chain. On the other hand, it is not clear in this strand of literature, how the definition of blockchain should depend on the dynamic interplay between these various approaches and dimensions.

Yet other works provide a more comprehensive, unified, and coherent overview of blockchain by focussing on its modular layers: the blockchain stack. There are various approaches and interpretation of the blockchain stack, all slightly different. Some authors merely distinguish 
between a "fabric" layer (enabling network transmission) and an "application" layer (Glaser, 2017). Many more identify three different layers, which are variously defined based on the approach adopted by the authors but that commonly distinguish between a hardware or network (infrastructural) layer, a protocol or consensus (platform) layer, and a data or application layer (Chu and Wang, 2018; De Rossi et al., 2019; Glaser et al., 2019; Neudecker and Hartenstein, 2018; Rauchs et al., 2018; Werbach 2018).

As remarked by many authors, blockchain is not a digital network per se, but represents an additional protocol, data, and application layer over the TCP/IP stack (see for instance De Filippi and Wright, 2018: 46-49). As a result, the blockchain protocol (the consensus rules) are often added as an additional layer of the stack and kept separate from the network protocol rules (network logic) regulating transmission and transactions among the nodes of the network (Gervais et al., 2016; Monegro et al, 2014; Rauchs et al., 2018; van Wirdum, 2016). For this reason, some approaches add extra-layers to the basic three, and sometimes they also including the external technological or social context (Ballandies et al, 2018; Notheisen et al, 2017).

The layered approach to blockchain provides a unified overview of the logical elements of a blockchain, but it does not try to understand how the different nature of these layers impact the way in which a blockchain should be understood. Most importantly, no model tries to understand how the dynamic interactions of these various elements impact the nature of the blockchain.

In this section, we provide a unified framework for the definition of blockchain in function of the framework developed in the previous section, and we discuss the conditions that make a digital network a blockchain based on the nature of the first-order dimensions. The following section will then discuss how the changing nature of these elements and their dynamic interdependencies affect the nature of blockchain and its most fundamental characteristic: its de/centralized nature.

\subsection{Defining Blockchain as Digital Network}

Based on the analytical framework developed in the previous section, we argue that any digital network becomes a blockchain whenever it addresses the following three necessary and sufficient requirements: 1 ) it enables for peer-to-peer transactions among users that are broadcasted to all nodes (NT); 2) it is used in order to transact social institutions in digital forms (i.e. digital representations of rights and value), here denoted a Coin (C), and is equivalent to data (D) in the general model in Figure 4.1; and 3) it does so by employing cryptographic algorithms in order to ensure at the same time the privacy (freedom to transact unhampered by surveillance, supervision and consent of political authorities) and the equality (identical roles and capabilities) in transactions of all its members vis-à-vis each other (social community SC). Figure 5.1 provides an overview of this and is unfolded in the following discussion. Consistent with the common understanding of a blockchain layered stack, it also adds a fourth element, the blockchain (cryptographic) protocol (CP), which operates as a logical intermediary between the various dimensions by ensuring their mutual consistency. 


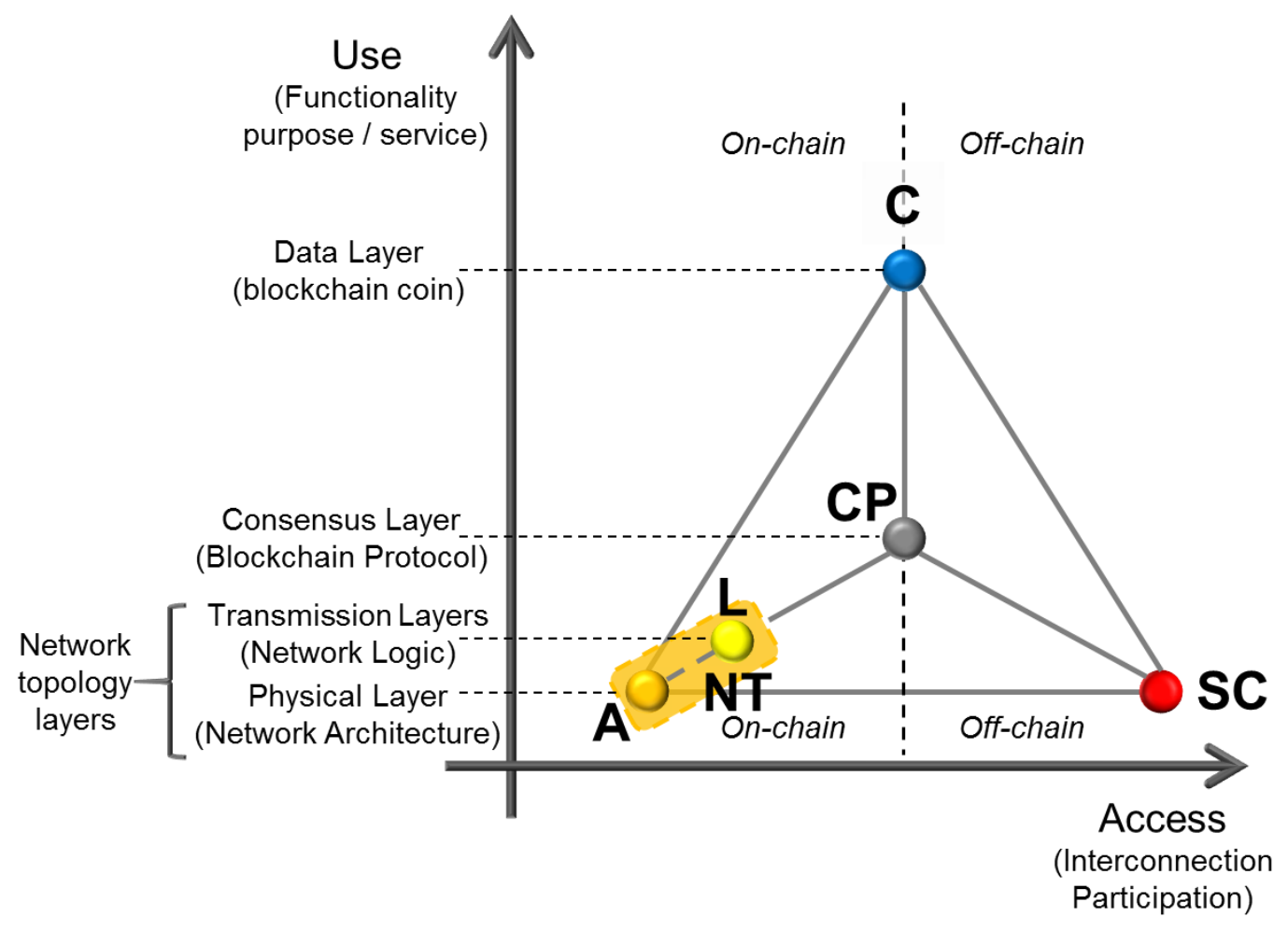

Figure 5.1: The general conceptual model translated to the context of blockchain with the addition of the consensus protocol (CP).

\subsection{1 (C) Coin: Data as Social Claims}

One of the most idiosyncratic features of a blockchain as compared to any other digital networks derives from the fact that the data transacted over it represent social institutions or, more specifically, claims valid against all other members of the blockchain.

While digital data can be indefinitely copied, duplicated or modified across libraries (digital data are, in principle, public goods), a fundamental characteristic of a blockchain is its capacity to forbid the double-spending of any digital data, and this means that transacted data in the blockchain is not copied and duplicated, but transferred. As noted by many other authors (Miscione et al., 2018, 2019; Ziolkowski et al 2018b), as a direct result, data on the blockchain ceases to be a public good, and becomes rivalrous and excludable among nodes. The capacity to enforce property rights in digital forms introduces scarcity in the digital world and ensures uniqueness of digital data. This also means that blockchain data not only have legal value as titles of ownership, but they also acquire economic value in exchange (monetary value). ${ }^{27}$

Money and property rights are social institutions and can both be intended as claims against society, or as rights. A claim against society is a claim that holds indiscriminately against all members of the social community regardless of the fact that any specific bilateral relationships between the title holder and any other member of the social community exists. It is an impersonal "right in rem" in

\footnotetext{
${ }^{27}$ As it has been effectively described by some authors, the blockchain provides the necessary digital infrastructure for the "internet of value", "internet 3.0", "Web 3.0", or an "internet of money" (Kewell et al., 2017; Swan, 2015; Tapscott and Tapscott, 2017).
} 
Hohfeld's (1913) terminology. In this case the society corresponds to the circle of members of the particular blockchain community holding and transacting the specific coin endowed with the agreed legal and economic significance. The shared or distributed ledger ensures that all claims enjoyed by all member of the community can be accessed and known to all other members of the social community, and is therefore ideally enforceable against the whole society (Arrunada, 2012, 2018). The autonomous and impersonal enforcement of these claims against all other members is the essence of a so-called "smart contract" (Werbach and Cornell, 2017) ${ }^{28}$.

As the symbolic meaning of the blockchain data is legal or economic, the symbolic dimension of a blockchain network should be understood adopting a legal and economic interpretative approach. As a result, while a digital data can in principle acquire a variety of symbolic meanings depending on its functionality and use as a bit of communication, content, video, information, and so on, the symbolic dimension in the case of a blockchain really has a legal or economic significance. The digital data (D) in Figure 4.1, is here in the context of a blockchain, a coin (C in Figure 5.1), or token, representing a title, a right, or in other words, a social claim endowed with economic and legal meaning. While blockchain coins were originally endowed with economic meaning and defined as "digital money", "virtual money", or cryptocurrencies (Birch, 2017; Gleeson, 2018; DuPont, 2019).

Starting from 2018, even the concept of cryptocurrency will be abandoned and replaced first by "token", then by the one of "asset" (digital or tokenised asset) (FCA, 2018), and finally by the much more general concept of digital right (property or contractual right) (US congress 2018 H.R. 7356) (See Blandin et al., 2019). This progressive conceptual movement away from the concept of currency or money came full circle with the conceptual shift from the original physicalist and metallist interpretation of the coin's economic value (Maurer et al., 2013; Swartz, 2018) towards a modern "legal" interpretation of exchange value (and money) intended as a redeemable claim (or right) on the social community or part of its members (Simmel, 2004; Dodd, 2014; Gleeson, 2018). The coin becomes socially valuable within the blockchain network as it represents a legal claim (right) against the members participating in the network.

\subsection{2 (NT) Network Technology: Interactions among Equal Nodes}

From a technological standpoint, a blockchain is a transparent p2p messaging system that allows each node to directly transact these titles with all the other nodes. Although there are various ways in which a $p 2 p$ system can be defined, we here adopt the most accepted interpretations and define a p2p network as a system where any node of the infrastructure can at the same time operate as a client of other nodes and as a server for other nodes (Tanenbaum and Wetherall, 2011: 6-10). As a result, $p 2 p$ is as much an architectural as a logical property of a digital network, which ensures symmetry in roles of all nodes over the blockchain. Because of the $22 p$ logic, each node is at the same time an enforcer of a claim on other nodes and subject to the claims of other nodes. Alternatively, each node is at the same time a seller and a receiver of a title. The peculiar nature of the coin transacted over the network also involves another peculiar feature of the logical aspect of the technological dimension of a blockchain and of its network protocol: all transactions changing the state of the network have to be broadcasted to the whole network and this ensures that the same copy of the blockchain can be accessed and shared by all nodes and that any claim or title can

\footnotetext{
${ }^{28}$ The concept of a "smart contract" was first coined by Szabo (1996).
} 
be known, accessed and enforceable by any node against any other node. This ensures on-chain symmetry in information among all nodes and makes the network logic of a blockchain different from standard $\mathrm{p} 2 \mathrm{p}$ networks where communication operates point-to-point, rather than broadcasted. ${ }^{29}$

While the symbolic dimension of the coin ensures that claims apply to the whole social community off-chain, the technological dimension ensures that these social claims are transmitted and known to the entire community on-chain. The network technology then defines the way in which various nodes can communicate and share information among themselves and the way in which transactions among these nodes occur.

A digital network where all nodes can play the same role within the network and where the information on the state of the system can be freely accessed and commonly shared and stored by all nodes with no distinction (so that the overall system behaves like a unique and coherent entity) is usually characterized as being distributed (Baran, 1964). As a result, from a technological (or network topology) point of view, a blockchain is de facto a distributed digital network, where all nodes behave as independent elements, but the system retains overall integrity and behaves as a unique system. This coherency and integrity is guaranteed by the common timestamp that also ensures that all nodes share the same common ledger containing the updated state of the claims enjoyed by each node vis-à-vis the others. Thus, while a blockchain is sometimes defined as a "distributed ledger", this is a narrow and partial understanding of the blockchain as it only characterises one dimension of the blockchain out of three: its technological one. Any blockchain is necessarily also a distributed ledger, but not just a distributed ledger.

\subsection{3 (SC) Social Community: Privacy (Liberty) and Equality in Transaction}

A blockchain is not just a shared collection of data among different nodes and a shared understanding of the rules governing transactions on-chain. It also represents shared ethical and political principles that inform and shape the nature of those rules and meaning of this data: liberty to transact unhampered by third-party consent or supervision, and the equality in treatment and social status among all participants (meaning that no party has a preferential role). These political ideals ensure that the validity of the social claims represented by the coin does not depend on the geographical boundaries of a State's rule of law (liberty), or by the peculiar institutional arrangement in force (equality), but only by the consensual agreements of the members of a social community. May (1994) calls these land-less and law-less communities where members are simply linked by "a shared ideology or particular interests" - in effect virtual communities. As a result, the third element (SC in Figure 5.1), the social community, represents the set of members sharing these moral and political principles and agreeing to abide by these rules of transaction automatically enforced by the blockchain protocol.

The moral and political ideals of liberty (and privacy), and equality emerged in different stages as they represent the two sets of normative principles inspiring the two main groups that conjointly contributed to the development of the bitcoin's blockchain.

\footnotetext{
${ }^{29}$ For a good discussion of the architectural consequences of traditional p2p file-sharing networks, see Chapter 7 in (Goldsmith and Wu, 2006).
} 
The first contribution came from the writings of crypto-anarchists and cyperpunks (Hammill, 1987; Hughes, 1993; May, 1988a; 1988b; 1994). Both these movements were inspired by anarcolibertarian ideals, and focussed on the role of cryptography as a way to enable individuals to freely interact, transact, and communicate with anyone they wish to, without an external entity or authority being able to identify, censor, interfere, spy, tax, or deny permission altogether. These ideals were shared by a set of close friends, mainly cryptographers, such as David Chaum (1981; $1982 ; 1988$ ) and by hackers, such as the Anonymous community.

The second contribution mainly came from computer scientists and coders, following the lead of Dai (1998) and Szabo (1997, 1998, 2001, 2003, 2005), and, eventually, Nakamoto (2008). While the first wave of writings mainly stressed the importance of cryptography in order to preserve privacy and liberty for all members of the social (virtual) community, the second wave of contributions tried to "operationalise" the crypto-anarchist ideals and to make sure that liberty and privacy did not come at the expense of equality. They did so by addressing the problem of how to design the right incentives mechanisms and protocol rules in order to make sure that privacy and liberty in transactions should not necessarily rely on central intermediaries validating and settling the transactions among unknown nodes on-chain. In sum, the work of this second wave of ideologues ensured that political freedom among members off-chain did not necessarily require asymmetries and inequalities among the nodes on-chain. This conundrum (in technical terms, the solution to the "byzantine general's problem" ${ }^{30}$ ) was solved by creating self-enforcing incentives to maintain the integrity of the network by endowing the coin with economic value (and making sure that the members agree on that) (Narayanan et al., 2016). As a result, the economic value of the coin is as much an end of the blockchain as it is a means to fulfil its political precepts: in order to solve the privacy-equality conundrum, one cannot be achieved without the other and vice versa.

The combination of these two set of contributions ensures freedom and privacy among all members of the social community on the one hand, and equality of all actors on the other hand. Both waves of contributions inform the nature of the other two dimensions - coin (C) and network topology (NT). But while the former mainly focussed on the ideological problems associated to the nature and content of the data transacted (what is being transacted), the latter took this problem further and mainly focussed on the ideological problems associated to the way in which the content should be transacted and the network should be designed (how this specific data is being transacted).

The motives for any social community to join a digital network can be driven by different reasons, such as geographical proximity, national identity, cultural background, social status, professional associations, behavioural routines, and a variety of other drivers. As a result, the social dimension characterising the interactions and interdependencies among the members of a virtual community can be understood and interpreted from a variety of perspectives. In the case of blockchain, the actors were originally motivated to join and develop the blockchain predominantly by the ethical, ideological, and political ideals and principles of classical liberalism (Locke, 1947; Mill, 1867) embodied in the blockchain. For this reason, the social dimension of a blockchain network should be understood and interpreted adopting a political and ethical perspective: the social interactions and bonds among the actors of the blockchain community should be understood as the political or

\footnotetext{
${ }^{30}$ https://en.wikipedia.org/wiki/Byzantine_fault
} 
ethical precepts informing both the nature of the coin and the way in which these transactions take place on-chain (protocol). Both coin and protocol respect these political principles and represent the way in which privacy and equality ideals offochain are reproduced on-chain and vice versa.

\subsection{4 (CP) Consensus Protocol: Holding Everything Together}

The three core elements of a blockchain are (See Figure 5.1): 1) the social community (SC) characterised by the political and ethical precepts shaping the interactions and interdependencies among its members; 2) the digital network and its topology (NT); and 3) the coin (C), which represents the social claims transacted over it. Any blockchain needs a consensus protocol (CP) providing the right incentives to ensure that all three dimensions can hold together and can interact in the right way.

Each blockchain is univocally associated to a cryptographic protocol and its native cryptographic coin - originally called a cryptocurrency. There is a multiplicity of consensus protocols adopting a multiplicity of cryptographic algorithms. ${ }^{31}$ Both the coin (C) and the blockchain protocol (CP) provide the necessary logical instructions to the nodes on-chain, and the right incentives to the members ofchain, to make sure that social claims can be transacted and enforced on-chain in a p2p manner while also respecting the equality and liberty (privacy) political principles.

As a result, both the coin and the blockchain protocol can be treated as interfaces between the interactions occurring among the blockchain nodes off-chain (offline) and the ones occurring among the members of the social community on-chain (on line). The coin represents digitised social claims transacted and enforced on-chain, but retaining their legal and economic validity among the members of the social community off-chain, while the blockchain protocol represents protocol rules defining the way in which different nodes can interact and communicate consistent with the political and ethical principles that also regulate the way in which the various members of the social community can interact and coordinate off-chain (Lessig (1999) "code is law"). The dual role of the blockchain's coin and protocol makes blockchain an institutional, rather than a productive, technology, as remarked by Davidson et al. (2018). Moreover, as digital data now represent social institutions with a non-modifiable ${ }^{32}$ meaning regulating incentives and social coordination among the members of the social community. The coupling between data and social community is now much more meaningful than in the case of standard digital networks, where digital data are perfectly reprogrammable, reproducible and editable.

More generally, the blockchain protocol can be understood as the fundamental logical intermediary between the three core elements of a digital network along both dimensions of access and use (See Figure 5.1 above). Firstly, the blockchain protocol regulates the way in which coins can be transacted

\footnotetext{
${ }^{31}$ Consensus algorithms, such as; proof-of-work, proof-of-stake, proof-of-authority, and proof-of-burn, apply a range of cryptographic algorithms, such as, SHA-256, X11, Dagger Hashimoto. The mechanics of these is not the focus of this document but can be studied elsewhere, such as in and so forth are discussed widely, for example by (DuPont, 2018).

${ }^{32}$ This is a different issue than whether or not blockchain arrangements are "tamper-proof." Once established, the agreement of what value the coin constitutes and by which means this is secured through the protocol, cannot be changed unless 1 ) the community as a whole decides so, or 2) the community split and continues in separate directions. The fact that blockchains are seeking to be tamper-proof but rather should be seen as tamper-resistant is a separate issue related to the ability to uphold the protocol-coin binding agreed upon.
} 
on-chain on-the-top of the network ( $\mathbf{C}<->\mathbf{N T}$ ) and accumulated and created by its nodes. Secondly, it regulates the on-chain/off-chain interfaces between the relationships among the members of the social community and their digital claims transacted, stored and enforced on-chain (SC <->C). Thirdly, it also represents the interface between the political rules of the social community off-chain and the topological rules of the network on-chain (SC <-> NT). In other words, the blockchain protocol provides overall coherence and consistency to the three dimensions of a blockchain. For this reason, it will also play a fundamental role in shaping the nature and the degree of de/centralization of a blockchain network, as discussed below.

\subsubsection{Blockchain as a Digital Network}

Based on the previous discussion, it is possible to modify our tripartite framework characterising digital networks as represented in figure 5.1. Differently from the standard framework for digital networks, both the blockchain protocol and the coin sit at the interface between the social interactions of the members occurring off-chain and the on-chain technological interactions of the network elements. The new element, the blockchain consensus protocol (CP) sit on the top of the network's physical and logical layers and operates as an intermediary and a "buffer" between the three elements, by regulating the way in which they interact and the way in which the characteristics of one dimension affect, and can be transmitted to, the other dimensions.

The vertical "use" axis in figure 5.1 reproduces the four typical layers of the blockchain stack. Some authors also add a fifth application layer (Monegro et al, 2014; van Wirdum, 2016), while others ignore the physical layer and only focus on the core three layers of network logic, consensus protocol, and data layer (Rauchs et al., 2018). Both the network transmission layers and the blockchain consensus protocol define the logic according to which the nodes and elements of the system can communicate and interact. But while the network logic regulates the way in which nodes can transact the coin among themselves, the consensus logic regulates the way in which the nodes are incentivised to verify, validate and append these transactions to the ledger, consistent with to the equality and privacy principles.

In conclusion, there are two ways in which a blockchain can be studied and understood. One way is to focus on the single elements and on the static first-order characteristics of the single dimensions, while an alternative way is to focus on the dynamic interactions and interdependencies among these dimensions. As we will argue below, a static characterization of the single dimensions and their firstorder interdependencies is only sufficient in order to define a blockchain, while an analysis of the dynamic second-order interdependencies between these dimensions becomes necessary in order to characterise a blockchain and understand its nature and degree of de/centralization.

\subsection{First-Order Blockchain Dimensions}

The decomposition of a blockchain into its core components shows that any digital network can be defined as a blockchain whenever all its fundamental dimensions are characterised by static firstorder symmetries among their respective elements. This does not mean that a blockchain is decentralized, but only that a digital network characterised by first-order decentralization for all dimensions is a blockchain. We will explore this aspect this aspect by considering each of the three first-order dimensions in turn. 


\subsection{1 (NT) Architectural and Logical A/symmetries}

The distributed logic that supports the $p 2 p$ architecture ensures symmetric interdependencies among all nodes from a topological point of view. The fact that any node can act at the same time as a beneficiary of a data transaction (recipient), or as a repository of information for other nodes (sender), generates interdependencies as any node is able to affect the others. The fact that the same set of information is broadcasted to all nodes and is identically stored by all nodes in the network ensures that these reciprocal influences are in principle identical, and therefore perfectly symmetric. Moreover, the way in which the consensus protocol was originally conceived also ensured perfectly symmetric capabilities among nodes in their ability to verify transactions and set incentives.

From a topological point of view each node is perfectly equivalent (and therefore symmetric) to any other node, consistent with the precept of privacy and equality. As a result, the technological dimension is characterised by first-order decentralization among its elements. This means that a breach of this symmetry implies that a digital network fails to be a blockchain. However, this does not mean that the blockchain is technologically decentralized: blockchain is a distributed system and, as such, it is logically centralized. Centralization in this case derives from the fact that all nodes share the same data within the common resource (the ledger) and cannot behave independently from each other. Logical centralization in this case emerges over time and derives from the fact that all nodes share the same distributed time stamp that logically links them all. As a result, while symmetry emerges as a static characteristic of the technological first-order dimension, asymmetries emerge in a dynamic setting, due to the way in which the various nodes can dynamically interact among themselves over time.

\subsection{2 (C) Legal and Economic A/symmetries}

The symbolic dimension is usually characterised by independence among its elements. Digital data usually do not generate interdependencies because of their public good nature: any data can be copied and reproduced, and therefore used independently from all others. Conversely, scarcity and rivalry always generate conflicts and, as a result, interdependencies. For this reason, differently from standard digital networks, the symbolic dimension of a blockchain is characterised by interdependencies among its data, meaning that the value or significance of one data depends on the value assumed by others.

The definition of data as social claims (titles of property) implies symmetric legal and economic interdependencies between the data belonging to the symbolic dimension. On the one hand, all rights or titles are, by definition, interpersonal institution that only makes sense in relationship to each other: any claim generate a duty on all other claims and vice versa (Hohfeld, 1913; 1917). For this reason, the value and nature of each claim can only be understood in relationship to all other claims. On the other hand, claims against society such as money or property rights are impersonal and universal, meaning that they equally affect all members of the social community indifferently. In the most basic case in which each coin is symbolically identical to all other coins (symbolic homogeneity or equality), interdependencies among coins are completely symmetric, in the sense that any coin is affected by the others in the same measure in which it affects the others.

From a legal and economic point of view interdependencies among homogeneous coins are always perfectly symmetric. As a result, the symbolic dimension of a blockchain manifests first-order 
decentralization from a legal and economic point of view. However, this does not mean that a blockchain is symbolically decentralized. The presence of a distributed time stamp introduces temporal (dynamic) asymmetries among coins transacted and enforced in different moments in time: the value and significance of newer coins depends on the value and significance of older ones. In other words, the blockchain cannot be reverted as a change in the value of past coins necessarily affects the value of more rent ones. Temporal asymmetries among coins ensure the tamperresistance of a blockchain and derive from the fact that the value and significance of any coin in a specific point in time cannot be understood independently from (depends on) the value and significance of past coins. Even in this case, symbolic centralization emerges over time and derives from the fact that all coins in a specific point in time share the same history that symbolically links them all. As a result, while the symbolic dimension of a blockchain is characterised by first-order symmetries among its coin for any specific point in time, symbolic asymmetries emerge in a dynamic setting, due to the way in which the various coins depend on the previous ones over time.

\subsection{3 (SC) Political and Ethical A/symmetries}

Finally, the political and moral precepts of liberty and equality ensure first-order decentralization among all members of the social community for two distinct reasons. In the crypto-libertarian and cypherpunk ideal, liberty can either imply pure independence whenever a member is not willing to engage in a transaction (liberty as a lack of coercion), or it implies symmetry in knowledge and reciprocal information whenever two members decide to interact (liberty as privacy in transaction). This second case implies that no member has any advantage over the other due to its greater knowledge of the state of the system. On the other hand, the definition of a cryptographic consensus algorithm ensures that, at least in principle, all nodes can play the same role and have the same capabilities in accessing, validating, verifying and appending a transaction. This implies that not only are all nodes symmetric when it comes to their role as repository of information ( $p 2 p$ symmetry), but also when it comes to their role as verifications, settlers, and validators of transactions (political symmetry). In all three cases (liberty as independence from transactions, liberty as privacy in transactions, equality in roles, status and capabilities), the social dimension of blockchain ensures symmetric interdependencies among all its members and therefore it manifests first-order decentralization from a political and ethical point of view.

The fact that the blockchain protocol ensures and enforces first order symmetries among the members of the social community does not mean that a blockchain is a politically decentralized system. The dynamic evolution of the value and nature of the claims over time and of the motivations and incentives shaping the decisions of the nodes can generate temporal asymmetries among the members of the social community over time. Similarly, to the other two dimensions, even in this case political centralization can emerge over time and derives from the status and capability of a member can evolve and change based on its past decisions. As a result, while the social dimension of a blockchain is originally characterised by first-order symmetries among all its members, social asymmetries emerge in a dynamic setting, due to the different ways in which the various members adjust and update their decisions over time. 


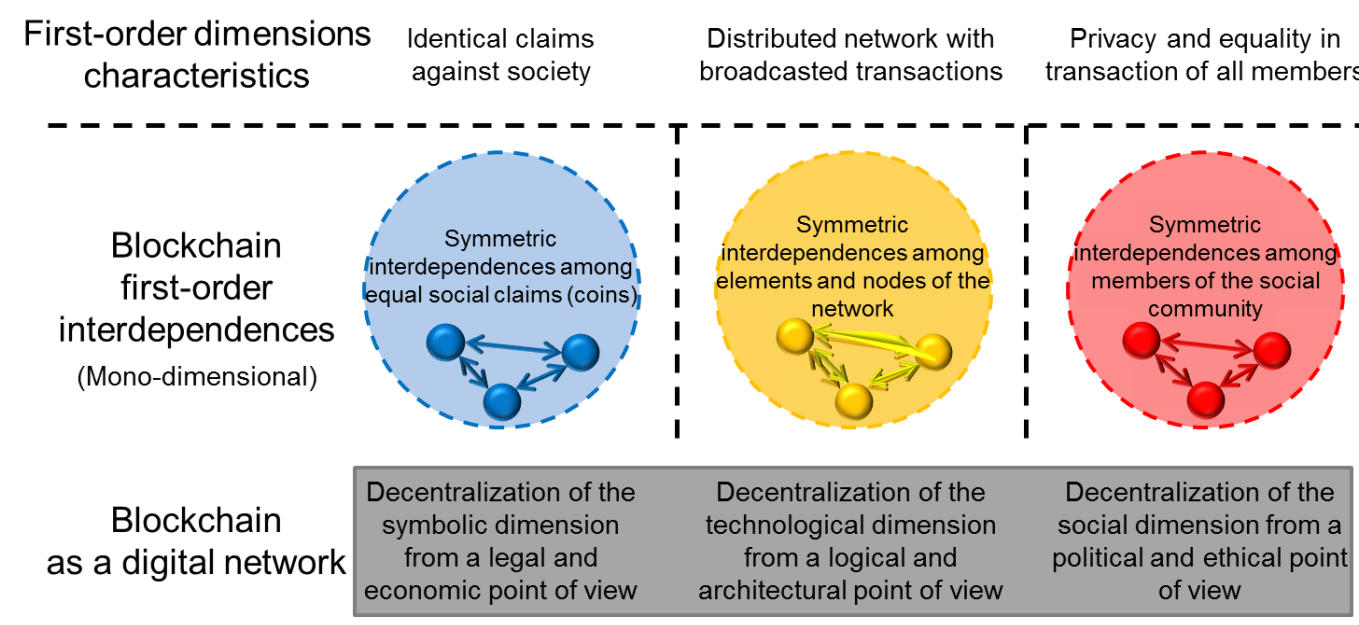

Blockchain as a first-order decentralised digital network

Figure 5.2: Characterization of the first-order dimension characteristics within Coin, Network Technology, and Social Community.

\subsubsection{First-Order Decentralization}

Based on our framework, we argue that first-order decentralization in all three dimensions of a digital network is both necessary and sufficient to characterise a blockchain. Whenever one feature is dropped, the same outcome can be easily replicated adopting a different, and potentially more efficient, technological solution. Tables 5.1 and 5.2 as well we Figure 5.3 provide overview of this discussion.

\begin{tabular}{|l|l|l|l|l|}
\hline $\begin{array}{l}\text { First-order } \\
\text { dimension }\end{array}$ & Unit & Approach & Characteristics of the element & $\begin{array}{l}\text { Alternative configuration in case } \\
\text { of asymmetry }\end{array}$ \\
\hline \hline Technological & NT & $\begin{array}{l}\text { Architectural / } \\
\text { Logical }\end{array}$ & $\begin{array}{l}\text { Distributed system: } \\
\text { P2p and Broadcast transmission of } \\
\text { transaction to all nodes }\end{array}$ & $\begin{array}{l}\text { Client-server database for exclusive } \\
\text { access to encrypted valuable data }\end{array}$ \\
\hline Symbolic & C & $\begin{array}{l}\text { Legal / } \\
\text { Economic }\end{array}$ & $\begin{array}{l}\text { Social claim: } \\
\text { Digitised institution representing a } \\
\text { claim against all members of the } \\
\text { social community (unique, non- } \\
\text { replicable, thus valuable) }\end{array}$ & Standard p2p file sharing systems \\
\hline Social & SC & $\begin{array}{l}\text { Political / } \\
\text { Ethical }\end{array}$ & $\begin{array}{l}\text { Classic liberalism “natural rights": } \\
\text { Privacy (liberty) in transaction and } \\
\text { equality in role and status among all } \\
\text { members of the community }\end{array}$ & $\begin{array}{l}\text { Two-sided market place run by a } \\
\text { two-sided platform characterising } \\
\text { the current platform (or sharing) } \\
\text { economy }\end{array}$ \\
\hline
\end{tabular}

Table 5.1: Overview of situations where one of the three necessary and sufficient first-order dimension blockchain definitions fail

The open internet has proven particular effective in the direct p2p exchange of data through file sharing protocols, such as, BitTorrent and eMule. Such $\mathrm{p} 2 \mathrm{p}$ file sharing software are fully distributed systems that can ensure the secure and potentially completely private $p 2 p$ transmission of encrypted data between two equal nodes. The use of encrypted and anonymised distributed systems has been historically regarded by hackers and crypto-anarchists as the only way to safeguard complete liberty and privacy on the web, while their distributed p2p nature also ensures 
complete equality and symmetry among all actors. ${ }^{33}$ Standard $p 2 p$ file sharing systems, however, cannot support the exchange of anything scarce as they cannot enforce property rights. They merely provide easy means by which data can be duplicated across a network of servers. For this reason, these files and data do not have any economic value in exchange. This is the case of a p2p community without a coin acting as an enforceable claim against the members of the digital community. This arrangement does not introduce rivalry among peers and for this reason it cannot generate on-chain symmetric interdependencies among the various data in the symbolic dimension.

Alternatively, it is possible to exchange objects that have economic value, such as paying for a second-hand piano on eBay. The $p 2 p$ transmission of valuable data that does not need to ensure privacy and/or parity of status of all nodes can be easily achieved through the operation of a central platform acting as a central intermediary in a symmetric p2p marketplace, where all nodes can be either clients or servers. Such preservation of value in exchange, however, necessitates trade through a central intermediary in charge for the validation and settlement of transactions: it is the well-known double-spending problem. In this case not only is the equality principle always violated, but the necessity to verify the validity of transactions also requires actors to trade in some of their private data. When purchasing an item from a merchant via Amazon.com in exchange for money, the interacting parties are not all equal. The central digital mediator, Amazon.com, has a different status than the surrounding buyers and sellers, and will, furthermore, become the owner of all data pertaining to the transaction and can potentially therefore subsequently monetise it (Zuboff, 2019). This is the case of a classic distributed business model, or digital market place, run by a two-sided (or multi-sided) platform, which characterises the current platform (or sharing) economy (de Reuver et al, 2018). In this case the first two requirements of a blockchain are respected, but not the third: the political and ethical preconditions of privacy and equality of all members operating over the infrastructure. For this reason, this solution cannot ensure symmetric interdependencies (from either an informative and structural point of view) among the various members of the social community.

The previous two configurations highlighted a choice between a situation of $p 2 p$ anonymity for the free sharing of plentiful digital objects, and one where members pay with their privacy when trading anything socially scarce and valuable, such as goods, money, or rights of ownership. Lastly is the case of a classic client-server database, where all members of a social community can request exclusive access to encrypted valuable data upon request from a common repository. This is the case of the access economy where claims and transactions are one-sided, rather than two-sided as in the case of $p 2 p$ marketplaces characterising the sharing economy. As the transaction is one-sided, there is no need to verify the legitimacy and validity of a transaction between unknown parties, so privacy can be ensured much more easily than in the case of a distributed two-sided configuration. By granting temporary and exclusive access to a protected and non-duplicable file or data, digital claims might be enforced, and privacy and equality of all members might be preserved, but the symmetric p2p architecture is substituted by the asymmetric hub-and-spoke one. Asymmetries in this case are both

\footnotetext{
${ }^{33}$ As summarised by Satoshi Nakamoto in one of its email exchanges with James A. Donald: "Yes, [You will not find a solution to political problems in cryptography] but we can win a major battle in the arms race and gain a new territory of freedom for several years. Governments are good at cutting off the heads of a centrally controlled networks like Napster, but pure p2p networks like Gnutella and Tor seem to be holding their own." (at https://satoshi.nakamotoinstitute.org/emails/cryptography/threads/1/)
} 
logic and architectural and, for this reason, this solution cannot ensure symmetric interdependencies among the nodes of the digital network in the technological dimension.

\begin{tabular}{|l|l|l|l|l|}
\hline $\begin{array}{l}\text { Multidimensional } \\
\text { systems }\end{array}$ & $\begin{array}{l}\text { Architectural and logical } \\
\text { decentralization of the } \\
\text { technological dimension }\end{array}$ & $\begin{array}{l}\text { Legal and Economic } \\
\text { decentralization of the } \\
\text { symbolic dimension }\end{array}$ & $\begin{array}{l}\text { Political and ethical } \\
\text { decentralization of the } \\
\text { social dimension }\end{array}$ & ( \\
\hline \hline Client-Server database & X & $\mathbf{V}$ & $\mathbf{V}$ & $\begin{array}{l}\text { Pre-Web } \\
\text { Web } 1.0\end{array}$ \\
\hline p2p file sharing & $\mathbf{V}$ & $\mathrm{X}$ & $\mathbf{V}$ & Web 1.0 \\
\hline $\begin{array}{l}\text { Multi-sided platform / } \\
\text { marketplace }\end{array}$ & $\mathbf{V}$ & $\mathbf{V}$ & $\mathrm{X}$ & Web 2.0 \\
\hline Blockchain & $\mathbf{V}$ & $\mathbf{V}$ & $\mathbf{V}$ & Web 3.0 \\
\hline
\end{tabular}

Table 5.2: Overview of the combinations of symmetry requirements across the technological, symbolic, and social dimensions.

\subsubsection{Summarizing the Framework}

In this section we have argued that a blockchain is a specific type of digital network characterised by the $\mathrm{p} 2 \mathrm{p}$ transmission of digitised (tokenised) social claims through the execution of a cryptographic protocol that ensures the equality and privacy (liberty) of all parties off-chain is also enforced and respected on-chain.

The previous three necessary and sufficient conditions retrace a common way to define blockchain in the literature as a: 1) distributed system, 2) for the transaction of digital value, and 3) employing a decentralized consensus protocol (or cryptographic algorithm). These three conditions are usually associated to the three modular layers of the blockchain stack (Rauchs et al., 2018: section 3; Blandin et al., 2019: section 2): 1) network layer, 2) data or application layer, and 3) protocol layer. However, some remarks are in order with respect to this understanding of blockchain.

Firstly, our framework shows that the use of the concept of decentralization to characterize a specific element or layer (the blockchain protocol) is not completely satisfactory and can be the cause of various misunderstanding and misleading conclusions. All layers and dimensions can be understood through the lenses of de/centralization (see also Rauchs et al., 2018: 44-46). When the present framework is adopted, blockchain does emerge as a decentralized digital network. However, decentralization should characterise all dimensions. We argue that a digital network becomes a blockchain only when decentralization characterises the first-order interdependencies among the elements for all dimensions, not just one.

Secondly, to merely focus on the nature and characteristics of the three digital layers on-chain overlooks the characteristics of the social dimension of a digital network and the role played by the members of the community off-chain. Consistent with the point above, a blockchain is a blockchain if and only if the social interdependencies among the members of the community can also be characterised as "decentralized".

Thirdly, and most importantly, we argue that any digital network is a system of systems, or a secondorder system. The identification of the characteristics that make each individual dimension decentralized is necessary and sufficient to characterise a digital network as a blockchain. However, it is only necessary and never sufficient to characterise the entre blockchain system as decentralized. Whenever a system is multidimensional, decentralization is not merely a function of the 
characteristics of the single dimensions, but a dynamic and relational characteristic of a system as a whole, which also takes into account the dynamic interdependencies between layers and dimensions (Schneider, 2003, 2019). As a result, the mere focus on the static symmetries characterising each dimension underestimates the degree of centralization of the entire system as it does not capture the asymmetries emerging from the dynamic interactions between different dimensions over time. In order to characterise a multi-dimensional system as decentralized, dynamic interdependencies among dimensions should be considered too.

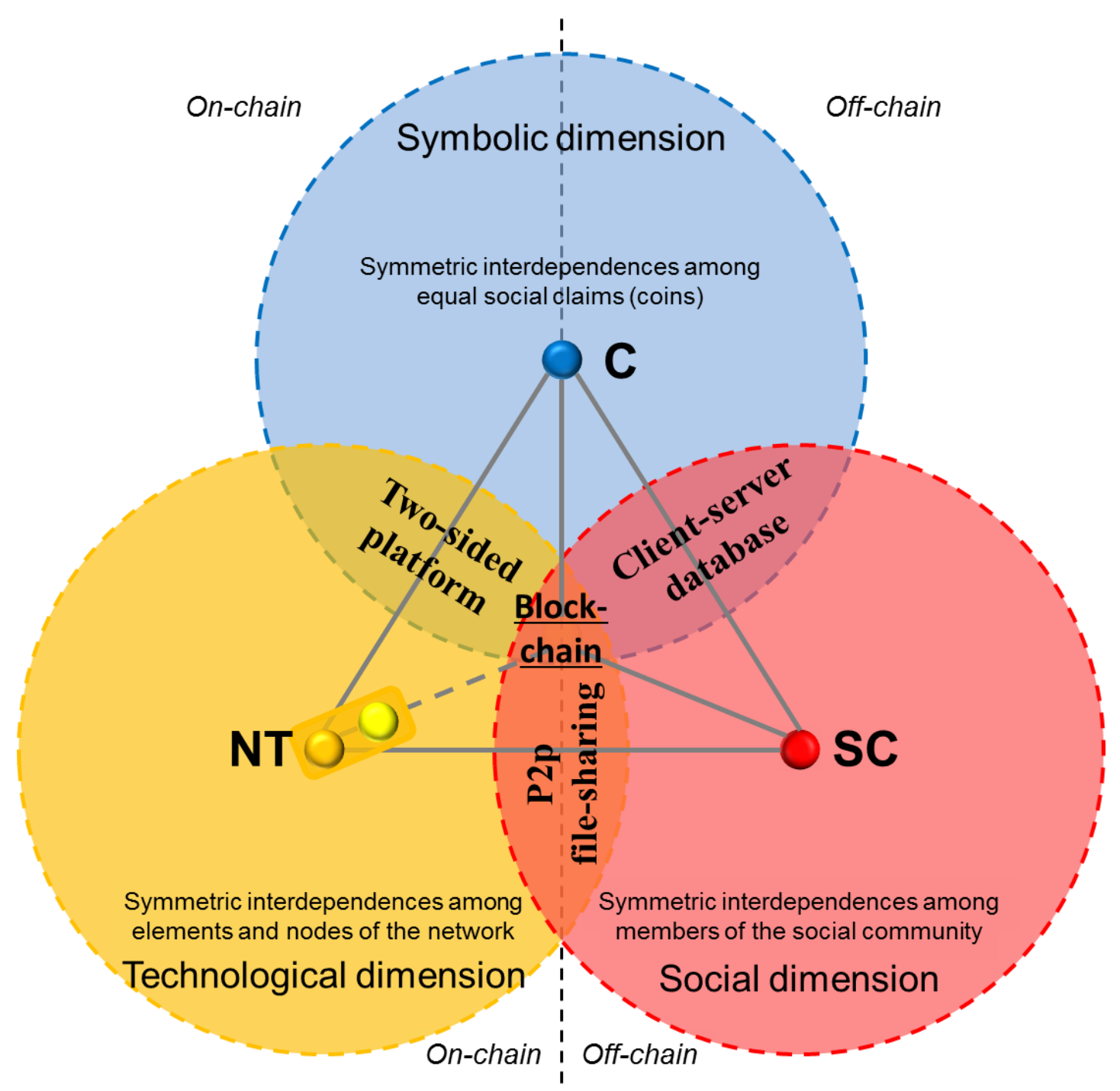

Figure 5.3: Topological overview of failures to establish symmetry on one of the first-dimension aspects

In the case of blockchain, this distinction should be understood as follows: the fact that a digital network can only be defined a blockchain if and only if all its first-order dimensions manifest static decentralization does not mean that a blockchain network is also decentralized. Stated differently, first-order symmetries in all dimensions of a digital network are a necessary and sufficient condition to conclude that a digital network is a blockchain, but we argue that they are only necessary and never sufficient to conclude that the blockchain is also decentralized. A blockchain is a term that 
should be used to characterise the static nature of a digital network (and its dimensions). Yet, a blockchain can be more or less de/centralized due to the dynamic (causal) interdependencies of its dimensions over time.

While it is true that the blockchain protocol represents the logical interface between the three dimensions, we argue that it is not the blockchain protocol per se to be more or less de/centralized, as it is frequently argued in the literature. Rather, de/centralization emerges from the peculiar ways in which the blockchain protocol shapes the dynamic interactions and interdependencies among these dimensions.

In conclusion, to understand whether a digital network can be defined a blockchain, it is necessary (and sufficient) to look at the characteristic of each first-order dimension, while in order to understand degree of de/centralization of a blockchain it becomes necessary (and sufficient) to analyse the degree of de/centralization across dimensions (horizontally and vertically). In order to do so, the focus of the analysis should be shifted from the interdependencies of first order within each dimension to the interdependencies of second-order across dimensions. This also implies a shift from the analysis of the static characteristics of each dimension to the analysis of the dynamic causal interdependencies between dimensions. 


\section{De/centralization and Blockchain Networks}

This section takes up the task of mapping and discussing de/centralization in second-order dimensions, i.e., dynamic a/symmetries between the three fundamental dimensions (symbolic, technological, and social) rather than static a/symmetries within each dimension of the first-order system. Figure 6.1 provides an overview of the interdependencies discussed.

\subsection{Second-Order Blockchain Symmetries}

The three dimensions of a blockchain assume three very specific analytical meanings and play three very specific roles within the broader blockchain system.

The technological dimension represents the locus of on-chain rules regulating and shaping the interactions among the various nodes; it is where the set of technical rules codified in the "code is law" formula operate, are implemented, and enforced. The technological dimension is a mix of architectural (spatial) and logical (procedural or organizational) rules.

The coin belongs to the symbolic dimension from where value and incentives come and where legal claims are defined. Depending on the nature of the institution represented, on the source of value, and on the nature of the claim. This dimension can assume a chiefly economic significance whenever the coin is purely intended as a means of exchange whose value is backed by social conventions. Alternatively, it can take on a legal significance if the coin is intended as a legal claim or title, which is enforceable against some other legal institution such as a court, a central bank, a government, and so forth.

The social community is the element embodying the political and ethical dimension ruling the agreements among the members of the real world. From an analytical point of view, it represents the normative precepts that inform the way in which the network rules and protocol are defined and interactions among the members of the social community occur. By sharing the same motivations and principles, the members of the social community establish the rules of the cryptographic protocol and agree on the necessary decisions facilitating the exchange of coins without the need to trade in private data, under the condition of equality and personal liberty. If the technological dimension represents the positive set of rules defining "code is law", the political and ethical dimension represents the normative precepts that inform the rule of code in the first place: "law is code" (De Filippi and Hassan, 2018; Reijers et al., 2018).

Given the significance of the three dimensions, it is possible to study the degree of centralization/decentralization of the blockchain by looking at how asymmetric dependencies in the form of powers and rights emerge and are shaped by the various patterns of interdependencies between these three dimensions. Differently from standard digital networks, second-order interdependencies in the case of blockchain do not merely emerge along the two "orthogonal" dimensions of access and use, but also along the new linkage between the coin and the social community. This third interdependency characterises the way in which value and incentives on-chain shape, and are shaped by, principles, beliefs, and agreements on value and incentives off-chain, and it is what distinguishes the blockchain from standard digital networks. 


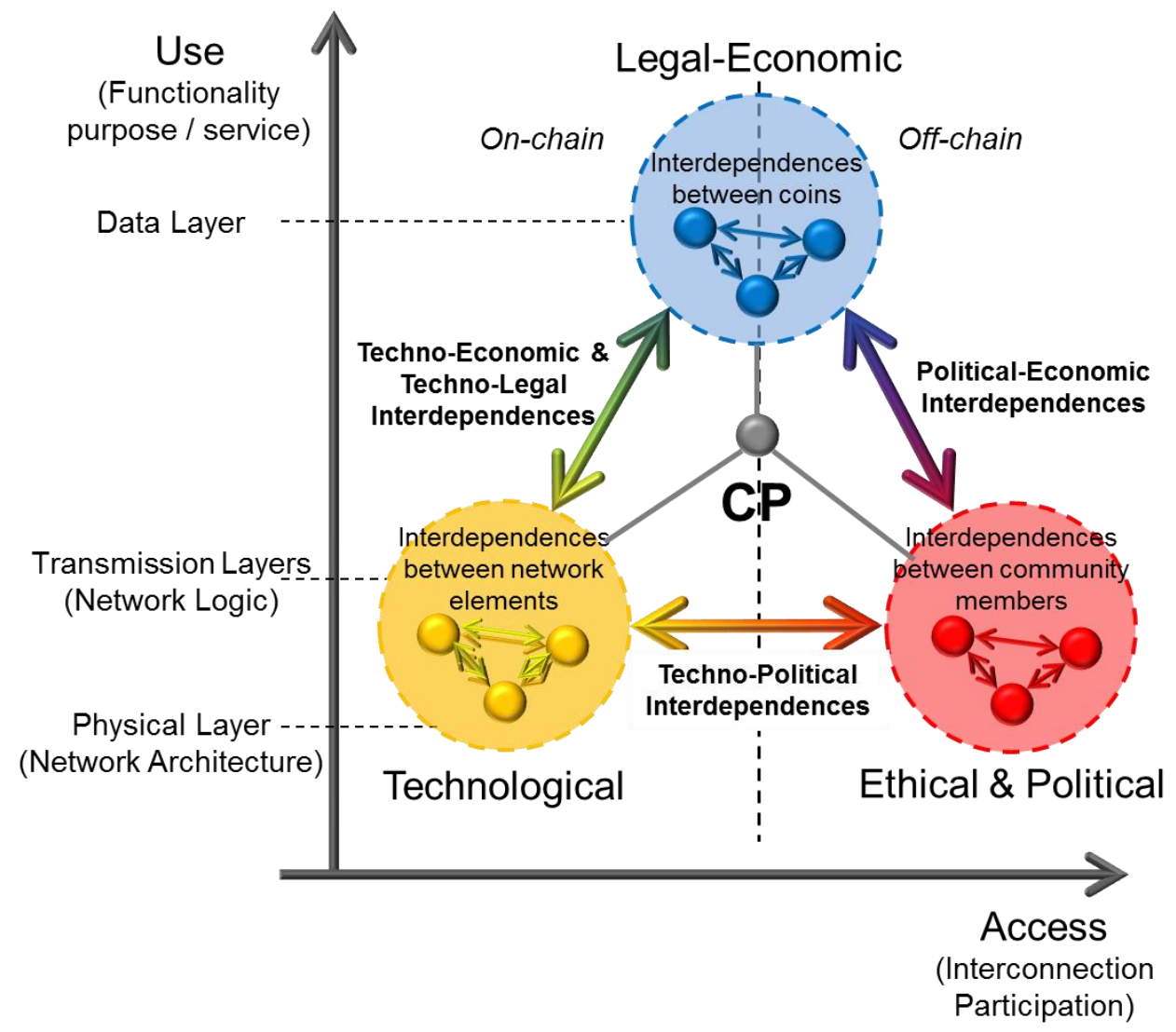

Figure 6.1: Illustration of the second-order interdependencies between the first-order dimensions.

As a result, three interpretations and sources of blockchain de/centralization can be identified, which we classify as follow (See also Table 6.1): 1) the political-economic (or political-legal) dimension of de/centralization focusses on the asymmetries emerging from the coin-social community relation in function of a given technological design; 2) the techno-economic (or technolegal) dimension of de/centralization focusses on the asymmetries emerging from the network-coin relation in function of a given political design; and 3) the techno-political interpretation of decentralization focusses on the asymmetries emerging from the network-social community relation in function of a given coin.

\begin{tabular}{|l|l|}
\hline Second-order interdependencies & Second-order asymmetries in rights and powers emerging from: \\
\hline \hline Political-Economic/ Political-Legal & Dynamic interdependencies between the coin and the social community \\
\hline Techno-Economic/ Techno-Legal & Dynamic interdependencies between the network technology and the coin \\
\hline Techno-Political & $\begin{array}{l}\text { Dynamic interdependencies between the network technology and the } \\
\text { social community }\end{array}$ \\
\hline
\end{tabular}

Table 6.1: Overview of the three second-order interdependencies

All these second-order interdependencies occur through the blockchain protocol that acts as a logical intermediary between the dimensions, regulating the way in which these can interact and affect each other. 


\subsection{SC $\Leftrightarrow$ C: Social Community and Coin}

As far as we have been able to trace, the first academic publication on Bitcoin was in 2013 following the revelations of the Silk Road on-line marketplace. This also marked the start of the academic debate on decentralization, and this debate was in the first couple of years focused exclusively on Bitcoin and not on the blockchain, which remained a little-known concept. This changed around 2015, which was the year the Ethereum infrastructure started its operations. Researchers started to enquire the meaning and nature of decentralization as applied to currencies: whether a virtual currency could be treated as money, whether a decentralized currency such as bitcoin could have any value if not backed by any legitimate institutions, where this value comes from (in case it did have some value), and how its decentralized nature could affect its value.

In other words, the first serious attempts to understand the concept of decentralization, to try to define and characterise its nature in the crypto-world, mainly centred on one element of the blockchain triad, the valuable coin. It did so mainly by treating the coin as an economic or financial tool. More specifically, the literature initially addressed the interesting problems raised by a decentralized currency. It did so by looking at the way in which the concept of decentralization is shaped and affected by the relationship between the coin and the social community using it. These first inquiries then adopted a political-economic (or political-legal) approach to define de/centralization that mainly looks at how the political precepts of the social sphere and its resulting institutional arrangements affect the significance of the coin and the nature and origin of its value. In other words, this first line of works tried to understand how the value of the various coins (and therefore their symbolic interdependencies) is affected by, and interact with, the specific interdependencies and relationships between the members of the social community.

Under this political-economic approach the technological dimension plays an important role but it often remains in the background. The network technology and protocol are usually given parameters of the problem that support and enable the interaction between the symbolic and the social dimension. Yet, technological considerations are usually taken for granted and rarely enters the analytical considerations of how centralization should be interpreted or how they might endogenously affect the very meaning of de/centralization. It is the de/centralized nature of the coin and the social origin and meaning of its value that spawned interest, not necessarily the nature of the underlying technology supporting its transactions.

If one looks at the way in which the literature in this branch developed, one will note that two different subsets of works can be distinguished: a "metallist" and an "informational" interpretation of decentralization. Both treat the coin as a fungible ${ }^{35}$ entity that can be used to conduct bilateral and private transactions and both try to understand how the value and significance of the single coins depend on social asymmetries among the members of the social community. However, the

\footnotetext{
${ }^{34}$ The term "metallist" has been chosen as the proponents generally subscribe to the notion of bitcoin as "digital gold" and sees the 1970s collapse of the gold-standard (The Bretton Woods Agreement) as negative since it resulted in inflationary national currencies and is discussed further in the following sub-section.

${ }^{35}$ The discussion of the extent a cryptotoken coin can be fungible is beyond the direct scope of this report. As all coins/tokens are enumerated, the coins are not fungible in a strict sense. However, the social community may still treat coins as though they are. Also, some arrangements, such as Zcash, allow completely anonymous transactions and thus rendering the coins fungible.
} 
two approaches adopt two different perspectives and two different interpretations of de/centralization.

\subsubsection{Metallist De/centralization: Coin as Digital Commodity}

The first approach defines a cryptocurrency as decentralized whenever it is neither governed, created, nor owned by any identifiable entity. In the physical world, this entity would be a central bank or a central government. In the crypto-world, this entity is probably a member of the social community or an identifiable organization claiming ownership over the coin. As a result, this interpretative approach defines decentralization by looking at the extent to which asymmetries among the members of the social community are reflected in the value of the coin for the whole community.

In this case a decentralized currency is characterized by the fact that no special member of the community is endowed with special powers or rights to own, control, or redeem the supply of the coin and therefore, by doing so, to directly and unilaterally affect its value for the whole social community. In other words, a decentralized system is one where the value and significance of the coin do not reflect the asymmetries in status, powers and rights among the various members of the social community. On the other hand, a centralized currency is characterised by the presence of a single actor (or a subset) endowed with the special powers or rights to directly control the economic value, supply, existence, validity and significance of the coin. These members would have the same powers and rights that central bankers or national governments enjoy.

In sum, the metallist interpretation of the political-economic approach to de/centralization looks at how asymmetries (inequalities) in the social powers, or legal rights, of the various members of the social community affect the dynamic evolution of the social value and significance of the coin (for all members of the social community) over time. In a decentralized system, there are no such powers or rights. These changes affect all identical coins and all members in the same (symmetric) way. Asymmetries therefore emerge either over time, or across coins. Whenever there are different coins appertaining to different communities, asymmetries in the social values and significance among the various coins emerge as a result of different asymmetric interdependencies among the members of the different communities.

The metallist interpretation of de/centralization looks either at the a/symmetries in the way in which the different preferences and wills of the various actors are aggregated at the social level and incorporated into the coin's value (focus on the political-economic aspect) or, alternatively, at the way in which single actors (or a group of actors) are responsible and liable for backing the value of the coin (focus on the political-legal interaction). The two correspond to, respectively, the political and the legal understanding of de/centralization provided previously. In both cases decentralization is understood along the lines of Austrian monetarists such as Hayek (1976), as a lack of central institution responsible to set (and back) the value of the coin on behalf of all other members of the social community. As highlighted by various authors, the independence of bitcoin from the control of socio-political institutions such as central banks and national governments represented precisely the fulfilment of the political and ideological goal of crypto-anarchists and Austrian monetarists consistent with Hayek (1976) writings (Ametrano, 2016; Corradi and Hofner, 2018; Dodd, 2018; Swartz, 2018; White, 2015). This is also consistent with the cross-border and trans-national essence of virtual social community. For this reason, the extra-legal and extra-institutional origin of value of a 
cryptocurrencies generated some interest in the economic and financial literature inquiring what could sustain the value of a coin that is neither backed by any social institution, by any commodity off-chain, nor has some intrinsically useful properties itself. ${ }^{36}$

Regarding the meaning of decentralization applied to a coin's value and nature, there is a difference between "setting the value" of a coin (mainly the political-economic perspective) and "backing the value" of a coin (political-legal). The members of a social community can agree that a certain coin has a certain value. However, in the absence of some recognised authority, they might be unable to back it up or ensure that this will always be the case regardless of the decisions and convictions of the participants. In the first case, decentralization along the symbolic-social linkage characterises the absence of asymmetric interdependencies among actors affecting the value of the coin. In the case of political-economic decentralization, the value of the coin remains socially-determined, even though it is not determined by a subset of unequal actors with special rights or powers. In a decentralized system the value of the coin must remain the indirect outcome of the collective and uncoordinated actions of equal and anonymous members of the community, not the direct outcome of a purposeful decision of one member (or group of members) of the social community endowed with special powers or rights over the coin itself. As symmetry in interdependencies work both ways, this also means that uncoordinated changes in the value of the coin provokes unplanned and uncoordinated changes in the way in which the different members of a social community interact among themselves. The metallist interpretation of decentralization represents Hayek's (1978) world of perfect competition applied to monetary theory. Any member of the social community can react and adjust its behaviour in response to a free-floating value and no special member can univocally and unilaterally determine this value based on its preferences or goals. In this case decentralization denotes a situation of symmetric interdependence between the value assumed by the coin and the interactions between the members in the social sphere, other than a situation of symmetric interdependence between different decentralized coins (floating exchange rate).

From a political-economic point of view, value in a decentralized system is then a social agreement among equal social actors. This does not explain what backs, enforces, and ensures the social value and significance of this coin. An important implication deriving from this peculiar approach is that a decentralized currency cannot represent a legal claim or liability of the owner against any specific entity as there is no special entity issuing, owning, or backing it. But this means that, while the value of a centralized currency can have a legal nature due to the presence of centralized institutions (it can be enforceable on all members as a legal tender), the value of a decentralized currency can never have a legal origin. The presence of a value rooted in the legal system would already denote

\footnotetext{
${ }^{36}$ The first works that started to inquiry the decentralized nature of bitcoin, decentralization focussed their efforts in trying to understand how the lack of control over the supply of coins would generate inherent volatility and instability of the coin's prices, and how this would affect the general welfare of society. (Ali, 2014; Ammous, 2018; Cheah and Fry, 2015; Li and Wang, 2017). Other works tried to understand and inquiry where the value of a decentralized coin comes from and how it should be understood (Cheah and Fry, 2015; Dwyer, 2015; Hayes, 2017; Li and Wang, 2017; Peters et al. 2015; Phillip et al. 2018; Schilling and Uhlig., 2018), whether a decentralized coin can be theoretically regarded as "money", or at least fulfil some the function of money (Ammous, 2018; Carney, 2018; Corbet at al., 2018; Corbet et al., 2019; Guesmi et al., 2019; Klein et al., 2018; Kubat, 2015), how decentralized digital currencies should be understood and what type of asset class they are (Burniske and Tatar, 2018; Dyberg, 2016; Nabilou and Prum, 2018; Wei, 2018), and how central banks and governments should respond (Barrdear and kumhof, 2016; Raskin and Yermack, 2016).
} 
some form of asymmetries and therefore of centralization of the monetary system. Decentralized cryptocurrencies are necessarily lawless coins and titles. The inherent lawlessness nature of decentralized currencies represents its core essence and one of the fundamental original motivations for their development as State-less and law-less currencies.

In the case of decentralized coins, the backing derives from the computer protocol running the network. As remarked by Swartz (2018), what was particularly appealing to the early cryptoenthusiasts was the fact that the supply and the creation of bitcoin is only governed by the protocol of the network, and is subtracted from the direct control of a central authority. This protocol-based value not only decouples value from the legal system, but it also gives the illusion that the coin has an intrinsic and a-social value, which only depends on the specific technology employed to generate it and run the system. It is the network itself and the rewards associated to the costly operation of its protocol that provide the backing for the value of the coin.

In the eyes of crypto-anarchist, a decentralized coin is therefore very similar to gold (or to commodity money): it has an intrinsic value that does not (necessarily) depend on the institutional and social context, but might only depend on its given scarcity, and on the effort or costs sustained in order to "mine" it. As a result, as noted by Swartz (2018), when decentralization is interpreted by adopting the crypto-anarchist perspective, the coin resembles more a store of (intrinsic) value (like gold) and less a currency (Ammous, 2018). Maurer and colleagues (2013) and Swartz (2018) call this approach "digital metallism" (see also Dodd, 2018; and Musiani et al., 2018). For this reason, we here define this peculiar political-economic understanding of decentralization in function of the coin-social community linkage the metallist approach to decentralization.

In conclusion, the metallist approach understands the centralization-decentralization dichotomy by looking at the extent to which the value of the digital coin derives from (and is dependent on) the peculiar nature of the social interdependencies among the members of the social community. Perfect decentralization implies that there is no central institution with the special rights or power to unilaterally redeem and enforce the social claim embodied by the coin on all members of the society, as this would generate asymmetries. Under this perspective, fiat money is always generating some centralization (especially if it becomes a legal tender) as its value among the members of the social community depends on the peculiar way in which social institution operate to back the value of the digital coin and ensure trust among the various members of the social community. In the case of fiat currencies, the nature of social interdependencies among the social actors off-chain does matter and has direct effect on the coin value. This situation denotes political-economic (political-legal) centralization as any change in the nature of the interdependencies among the members of the social community (such as a change in the power or legitimacy of a particular member in guaranteeing trust) necessarily also affects the value of the coin transacted and its social significance.

As a result, the symmetric interdependencies between the social and the symbolic dimensions can explain how value is set, but not how the coin can retain any value at all, or what gives the coin any binding force within the social community.

Decentralization from a political-legal point of view characterises a situation where not only no individual (or group of individuals) has the power to unilaterally affect the value of the coin, but where the value of the coin does not depend on the specific nature of social interdependencies at all. In a metallist perspective, decentralization does not merely require symmetric second-order 
interdependencies between the symbolic and the social dimension, but independence between the two dimensions. The absence of any legitimate institution able to back the value of the coin and to enforce it over the whole community (as a claim against society) implies that the origin of the value of the coin must be independent from the social dimension altogether. For this reason, a decentralized coin can retain any value only if it is understood as a commodity-money.

In the extreme metallist ideal, a decentralized cryptocurrency is characterised by the fact that the value of the coin is only "intrinsically" determined by the given technological dimension that supports it. According to this perspective, commodity-money is always decentralized, while fiat money will always generate a causal dependence of the coin from the peculiar interdependencies of the social dimension. This is also consistent with the anti-inflationary stance of Austrian monetarism regarded as a precondition of liberty and equality along the lines of Hayek (1978).

This also implies that in case of a decentralized coin there is little need for any mechanisms ensuring trust among the members of the community, as the only form of trust that is really needed is the one in the technology running the system, which also provides the ultimate backing and enforcement of the social claims embodied in the coin. The nature of trust supporting the value of the coin in the metallist approach is the trust in the correctness and reliability of the code. This is the essence of the so-called "trust-less trust" (or decentralized trust) of decentralized currencies. As the metallist understanding of de/centralization is only interested in disentangling the linkage between the social and the symbolic dimensions, all outstanding issues emerging from the problematic relationship between the technological infrastructure and the social community are completely neglected at this stage (and relegated to the techno-economic interpretation).

Bitcoin is the most obvious example of a decentralized coin from a metallist point of view: it behaves like a scarce commodity whose supply (and intrinsic value) is regulated by the cryptographic protocol run by the network. Its commodity-money nature derives from the fact that it is neither minted nor owned by any authority and, as such, no special member of the social community can redeem it or back its social significance. On the other hand, Ripple's coin XRP is usually accused of being a centralized cryptocurrency by crypto-metallists as the coin depends on asymmetric relationships within the social dimension: the coin is owned and can be redeemed by a central authority. For this reason, XRP is also more similar to a fiat currency (a pure means of exchange) rather than a commodity-money endowed with some a-social intrinsic value. The same is true for cryptoassets released through ICOs as their value can be controlled by a central authority that is also responsible for their creation and redemption. Finally, stable coins such as that proposed by the Facebook-led consortium Libra, are much more similar to fiat money as they are anchored to the value of other fiat currencies that, in the metallist view, are centralized forms of payment. Centralization in this case derives from the fact that their value directly depends on the decisions of the consortia or organization releasing them and deciding the benchmark they should be anchored to (and therefore directly setting and influencing their value). However, some findings suggest that the price of bitcoin might be strongly correlated to the price of the stable-coin Tether (Griffin and Shams, 2019; Wei, 2018), thus implying that the value of bitcoin might be substantially backed by the US Federal Reserve, as any other fiat money, and far away from the metallist ideal of bitcoin as a decentralized (in the sense of un-backed) digital commodity. 


\subsubsection{Informational De/centralization: Coin as Digital Fiat Money or Social Claim}

In the ideal world of cryptoanarchists and Austrian monetarists, perfect decentralization along the second-order coin-community interaction dimension can only be achieved by a coin behaving like a deflationary commodity: intrinsically useful, transnational, whose value is only dependent on asocial characteristics of the technological and ontological context. For these reasons, among the three or four ${ }^{37}$ key characteristics of money (Ali, 2014; Birch, 2017), a decentralized coin becomes a store of value, similar to gold. Decentralization in this case denotes a situation where the coin value is independent from the specific and contingent institutional arrangements of a particular social community and from the specific (asymmetric) status of some of its members.

According to the metallist definition of decentralization, only bitcoin and all cryptocurrencies following the original Proof-of-Work (PoW) consensus protocol can fit the definition of a purely decentralized coin. The fierce debate over the bitcoin block size and its scalability that led to the fork of bitcoin in 2017 showed that not all bitcoin enthusiasts intended the decentralized nature of bitcoin in the same way cryptoanarchists and Austrian monetarists did (De Filippi and Loveluck, 2016; Hsieh et al., 2018; Walch, 2018). Although much of the debate focussed on the problems associated to the centralization of the nodes on-chain (see the techno-economic approach), the quarrel also revealed a different understanding of bitcoin: advocates of smaller blocks intended bitcoin more as a means of exchange rather than a commodity for the store of value (Swartz, 2018).

As a result, the other way in which decentralization can be understood and defined along the coincommunity relationship is by considering the informational content of a coin qua means of exchange. Differently from the metallist interpretation, the informational approach does not define de/centralization by looking at the origin of value. The informational approach characterises the de/centralization of fiat money with a social value, rather than commodity money with an intrinsic value. The degree of centralization of a cryptocurrency in this case can be understood in function of its privacy-preserving characteristics and on its discriminatory properties. This interpretation of decentralization appealed especially hackers (such as Wikileaks members) and software developers, all particularly concerned with the threat that intermediation poses to individual privacy and freedom of expression (Swartz, 2018).

Consistent with the political-economic approach adopted by digital metallism, the informational approach also understands the degree of centralization of a currency by looking at the way in which asymmetries characterising the social community affect the value of the coin transacted and, more generally, generate symbolic asymmetries among the coins (and vice versa). However, differently from the metallist case, the interdependencies between the coin's value and the social community are interpreted differently, and so is the concept of de/centralization.

While in the metallist approach a decentralized coin becomes valuable because it is somehow intrinsically useful, deflationary, and intrinsically scarce, in the informational approach the coin becomes valuable to the extent in which it can convey the strictly necessary amount of information

\footnotetext{
${ }^{37}$ Money serves the purposes of: 1) a unit of account - can be measured; 2) a medium of exchange by being a commonly acceptable currency in exchange for goods or service; 3) a store of value as can be held as an investment and contain value as a result; and as added by Birch (2017), 4) a means of deferred payment supporting contractually arranged provisionf or future payment even subject to inflation or other changes.
} 
to the counterpart in order to enable trade and social coordination, while preserving privacy and freedom of transaction and communication. In the same vain, the decentralization of the coin is not interpreted as a way to tackle the special status of the central bank or central government and their legal monopoly over its supply. A "decentralized" coin mainly becomes a tool for free, open, disintermediated, and privacy-preserving social coordination among independent and equal members of the community. In this case, coordination, open access, and social interdependencies are valued above independence and a-social autarky fulfilled through the personal accumulation of an objectively and intrinsically useful commodity.

In the informational approach value is not univocally linked to the mechanism regulating the supply and creation of the coin (and the control over it), but it is linked to the informational content of the coin: it depends on the opportunities and options in which the coin can be used and which can be claimed by the coin holder while the coin is used as a means of exchange. The coin then plays a dual role: on the one hand it conveys information about what is possible and what opportunities are available to its holder before it is used and, and on the other hand, the specific way in which the coin is used by a member also reveals information about the coin holder status and preferences to the other members of the social community and in relation to them. The former defines the fungibility of the coin as a means of exchange, the latter defines the neutrality of the coin or, in other words, its capacity to discriminate one member from the other and to generate asymmetries among these.

The coin is therefore a dual informational tool endowed with informational value and for this reason we call this second political-economic approach the "informational interpretation". As a result, under the informational approach the distinction between centralization and decentralization becomes as follow.

In a decentralized system all actors transact the coin under the same informational setting and the coin has the same symbolic significance of fungible and neutral cash for all members. On the one hand, the coin has the same informational value for everyone (neutrality) and provides the same opportunities and capabilities to anyone (fungibility) regardless of how it is spent and who spends it: no special actor can obtain more value and enjoy more opportunities than the others while exchanging the coin. No subset of actors can benefit from unilaterally affecting the way in which others can use the coin. For this reason, in a decentralized system the symbolic value of a coin is perceived identically by all actors of the community independently from their relationships and asymmetric interdependencies, and no member can decide and constraint how others can use the coin. At the same time, all identical coins are symbolically indistinguishable, and this means that they are endowed with the same informational value, provide the same opportunities, and convey the exact same information to all social members (absence of discrimination). For this reason, in a decentralized system the symbolic value is identical for all identical coins, in the sense that all identical coins are endowed with the same symbolic content.

From an informational stand point, a coin is decentralized whenever second-order symmetries between the symbolic and the social dimension emerge: symmetries in the social dimension are reflected into symmetries in the symbolic dimension and vice versa. These two conditions reveal the dual nature of symmetries characterising the coin-social community relationship: decentralization in the informational approach implies symmetry in the value of a coin among all members (social symmetry) and symmetry in value among all identical coins (symbolic symmetry). The first implies 
symmetries in the social sphere: no actor has the right or power to benefit more from the transaction of a coin more than others, and therefore to benefit from it in an asymmetric fashion. The second implies symmetries in the symbolic dimension: no coin is special and is endowed with more (or less) informational value than all other identical coins. For this reason, it is not possible to discriminate among identical coins simply based on who employs them or how they are employed.

In conclusion, while a decentralized coin in the metallist interpretation is an intrinsically useful commodity endowed with a-social value (coin-community independence), a decentralized coin in the informational interpretation is a neutral and fungible means of exchange (or social claim) endowed with the same social value for all its users (coin-community symmetric interdependence), such as fiat money or cash. Differently from the metallist case, informational decentralization cannot, however, explain or justify what backs the value of the coin and what guarantees that this does not collapses to zero.

Conversely, a centralized system is characterised by informational asymmetries in the coincommunity relationship, which work both ways. Centralization can be understood as a situation where the informational content generated by any transaction of the coin spreads unevenly among the various members of the community. In this case, asymmetries derive from the fact that the symbolic significance of the coin (its informational content) is higher for some members than for others. This means that the value of the coin is higher for some members than for others: some benefit more than others from a transaction of the coin as a result of the fact that they know more than others.

This informational imbalance among the various members of the community reveals some form of (asymmetric) social power among the various members and the potential capability of these powerful members to control or purposefully shape the value of the coin at their advantage. In virtue of their social status or identity some members can extract more value from a coin than others, meaning that the coin is not neutral and offers an asymmetric set of opportunities or options to its different users. In this case, the fact that some actors have the privilege to better understand the social significance of a coin than others violate the equality principle of the social dimension and therefore the symmetry among all members of the social community. The same is true in the case of legal claims: having more information of who is exercising some claims and why can put some members in a position to influence the significance and the value of these claims.

In all these cases, some members might have a better overview or understanding of the value of the coin (or significance of the claim) compared to the other members of the social community in virtue of their special status within the community. As a result, some members with a better understanding of the symbolic significance of the coin might be able to purposefully and asymmetrically affect the value of the coin for other members.

On the other hand, asymmetries can operate the other way around: asymmetries in the symbolic dimension can be used in order to discriminate among members and therefore to generate asymmetries in the social dimension. In this case informational centralization characterises a situation where some coins can be distinguished and discriminated from others even though they are seemingly identical. In this case different coins have different informational value than others and for this reason they present different opportunities and fungibility to their holders. Asymmetries among coins in the symbolic dimension can be used to discriminate one member (coin holder) from 
the other: the social community is able to discriminate against some of its members by discriminating against some coins.

A typical example is one where a part of the community decides that certain coins identified by certain identifiers or serial numbers should be discriminated against, and therefore be regarded as "inferior" or "superior" social claims as compared to the other originally identical coins. The goal of this discrimination is clearly to place the holders of these coins in different social positions vis-à-vis the others members of the social community. Asymmetries in the symbolic dimension are used to purposefully generate asymmetries in the social dimension.

\begin{tabular}{|c|c|c|}
\hline & Metallist Approach & Informational Approach \\
\hline & \multicolumn{2}{|c|}{ Decentralization } \\
\hline $\begin{array}{l}\text { Meaning of } \\
\text { decentralization }\end{array}$ & $\begin{array}{l}\text { Interdependence between the symbolic } \\
\text { dimension and the social dimension }\end{array}$ & \begin{tabular}{|l|} 
Symmetric interdependence between the \\
symbolic dimension and the social dimension
\end{tabular} \\
\hline $\begin{array}{l}\text { Condition for } \\
\text { decentralization }\end{array}$ & $\begin{array}{l}\text { Socio-symbolic Independence: the coni can retain } \\
\text { an intrinsic value independently from the } \\
\text { contingent arrangements and interdependencies } \\
\text { in force among the members of the social } \\
\text { community }\end{array}$ & $\begin{array}{l}\text { Symbolic and social symmetries: identical coins } \\
\text { are endowed with identical claims (fungibility of } \\
\text { the coin) and the same coin gives identical claims } \\
\text { to any member (privacy and equality) }\end{array}$ \\
\hline Causality / Directionality & $\begin{array}{l}\text { Absent or } \\
\text { from the symbolic dimension to the social }\end{array}$ & Bidirectional / Symmetric \\
\hline Nature of the coin & Digital commodity (commodity money) & $\begin{array}{l}\text { Digital social claim (fungible and neutral fiat } \\
\text { money such as cash) }\end{array}$ \\
\hline Nature of value & Objective and a-social & Objective and social \\
\hline \multirow[t]{2}{*}{$\begin{array}{l}\text { Implications for the social } \\
\text { sphere }\end{array}$} & Lack of central banks or government & $\begin{array}{l}\text { Lack of identification-based (account-based) } \\
\text { payments }\end{array}$ \\
\hline & \multicolumn{2}{|c|}{$\begin{array}{c}\text { Centralization } \\
\end{array}$} \\
\hline Meaning of centralization & $\begin{array}{l}\text { Interdependence of the symbolic dimension from } \\
\text { the social dimension }\end{array}$ & $\begin{array}{l}\text { Asymmetric interdependence between the } \\
\text { symbolic dimension and the social dimension }\end{array}$ \\
\hline $\begin{array}{l}\text { Condition for } \\
\text { centralization }\end{array}$ & $\begin{array}{l}\text { Social interdependencies among social members } \\
\text { driven by social claims }\end{array}$ & $\begin{array}{l}\text { Symbolic and social asymmetries: Asymmetric } \\
\text { claims among identical coins (no fungibility or } \\
\text { neutrality of the coin) and among actors (no } \\
\text { privacy or equality) }\end{array}$ \\
\hline Causality / Directionality & From the social to the symbolic dimension & Bidirectional / Asymmetric \\
\hline Nature of the coin & $\begin{array}{l}\text { Digital social claim (fiat money backed by a social } \\
\text { institution) }\end{array}$ & \begin{tabular}{|l} 
Individualised / personalised / specific digital \\
social claim (voucher, ticket or coupon)
\end{tabular} \\
\hline Nature of value & Objective and social & Subjective and social \\
\hline $\begin{array}{l}\text { Implications for the social } \\
\text { sphere }\end{array}$ & Presence of central banks or government & $\begin{array}{l}\text { Presence of a social tool for members' } \\
\text { discrimination and identity revelation }\end{array}$ \\
\hline
\end{tabular}

Table 6.2: Summery of differences between decentralization and centralization in terms of the metallist and informational perspective on the coin-social community second-order dimension interdependencies.

The history of cryptocurrencies is abundant with these kinds of coin-community asymmetric interdependencies where symbolic asymmetries have been used purposefully and instrumentally in order to discriminate against certain members. Standards examples of this case are the decision to invalid certain coins that have been stolen or counterfeits in order to punish those holders that broke a social rule, or a decision to put some coins out of circulation as they belong to an old social order that is not recognised any more (for instance a fork). The case of the hacking of the Distributed Autonomous Organization (The DAO) investment fund is a very well-known example (Zachariadis et al, 2019). Here, the Ethereum community and its funders decided to invalidate and nullify the value of all the stolen coins. In this case, discrimination among coins was operated in order to generate asymmetries among different members of the social community (the hackers vs. the others): while some coins still represented valid claims against the Ethereum society, others could not be enforced as social claims, thus creating a duality in the social meaning and value of the same coin. Eventually, 
this duality also generated a fork (split) in the social community, each coalescing around a different social significance of the coin. In all these cases asymmetric symbolic meanings among the coins reflect social asymmetries among coalitions of members of the community and in the authority of certain members or institutions over others. A discrimination of the social meanings of the various coins necessarily benefits some members at the expenses of others or, vice versa, penalizes some compared to others.

In conclusion, while a decentralized coin in the informational interpretation is something like cash or a banknote (it is neutral, fungible, and retains the same value for all its holders), a centralized coin in the informational interpretation is more like a voucher or a personalised ticket or coupon: it allows to discriminate among members as it attaches to a specific member (it is not neutral as its value depends on the specific identity of the holder), and/or it can only be employed for a specific subset of purposes or uses (it is not fungible). Table 6.2 summarizes the differences between decentralization and centralization in terms of the metallist and informational perspective on the coin-social community second-order dimension interdependencies

\subsection{NT $\Leftrightarrow$ C: Network Technology and Coin}

Soon after the first works discussing the decentralized nature of bitcoin and cryptocurrencies, and contextually with the first conceptualization of Ethereum in 2014 (Buterin, 2014; Wood, 2014), the literature started to realise that bitcoin could just be one possible coin exchanged on the blockchain and one possible use case among others. This was realised as the attention gradually shifted from the economic nature and significance of the coin to the role of the underlying network technology in enabling the "trustless trust" of decentralized currencies or, in the case of digital metallism, their "society-less value". Starting from 2015, an increasing number of publications and reports conclude that the essence of a blockchain does not depend on the characteristics of the coin transacted over it, but on the underlying network technology enabling its transactions and supporting its value and meaning (Economist, 2015; Underwood, 2016; UK Government Chief Scientific Adviser, 2016; Tapscott and Tapscott, 2016). The focus shifts from the interdependencies between the coin and political rules of the social community to the interdependencies between the coin and the protocol rules of the network technology.

Considering the second-order interdependencies illustrated in Figure 6.1 above, it possible to identify six different interpretations of decentralization in the second-order interdependencies between the two first-order systems of Coin (the legal-economic system), and Network Technology (the technological first-order system). These six approaches fall within two distinct categories; a techno-economic, and a techno-legal approach:

\section{Techno-economic}

1) protocol-logic: the decentralized protocol as a risk-free lender of last resort;

2) permission-logic: the decentralized protocol as a neutral validation mechanism;

3) market-power: the decentralized network as an egalitarian distribution of capital;

\section{Techno-legal}

1) platform-logic: the decentralized network as a neutral and open general-purpose technology.

2) code-is-law: the decentralized network as an on-chain governance mechanism;

3) principal-agent: the decentralized network as coordination mechanism for the on-chain division of labour. 
In the following we will briefly contextualize these six perspectives before the following six subsections discuss each in turn in more detail.

In all six perspectives the concept of de/centralization is used to define the network rather than the coin. De/centralization ceases to define the coin and the decentralized nature of its value and starts to define the underlying network and the decentralized nature of its technology. De/centralization becomes a characteristic of the technological dimension, rather than a characteristic of the symbolic dimension: from cryptocurrencies as decentralized currencies (or money) to blockchain as a decentralized technology.

The expression "network technology" is quite broad and refers to various important aspects that are useful to distinguish. The two components of a network technology, its logic and architecture, perform two fundamental roles as they define: 1 ) the way in which coins can be transacted among nodes; and 2) the ways in which these transactions are verified and validated by the network, and subsequently appended to the common ledger. The characteristics of the network topology strictly defined directly deal with the former, while the ethical and political precepts of the social dimension inform the way in which the blockchain cryptographic protocol carries out the second set of activities (verification, validation and updating). Thus, the original bitcoin blockchain is characterised by $p 2 p$ transactions among nodes and by a cryptographic protocol that ensures equality and privacy of all nodes not only off-chain, but also on-chain in the validation and verification process.

The techno-economic and techno-legal interpretations of de/centralization recognise that the value of the coin is endogenously shaped by both aspects of the network technology through a change in the way in which: 1) the nodes can communicate and transact on-chain and directly affect the economic value and significance of the coin, and 2) the various nodes can verify, validate, and append transactions with direct implications for the economic value and significance of the coin. The interdependencies might be symmetric so that the reverse also holds: transaction and validation rules can be directly affected by the specific value assumed by the coin and by its symbolic meaning and significance.

Although the three approaches following a techno-economic interpretation of de/centralization, and the three approaches following a techno-legal interpretation share the same general view with respect to the interdependencies between the symbolic (Coin) and the technological dimension (Network Technology), each approach focuses on one specific aspect of this interaction.

First of all, these six different approaches can be distinguished based on whether the focus is placed on the coin or on the network technology. Some approaches study centralization by looking at the way in which the de/centralized nature of the network technology shapes the value and significance of a coin, while others study de/centralization by looking at the way in which certain values and significance of the coins shape the degree of de/centralization of a network. Obviously, both mechanisms can play a role even though each approach usually only focuses on one specific causality.

Secondly, these six different approaches can also be distinguished based on whether the coin is interpreted as an economic or a pure legal entity. The first group of works belong to the politicaleconomic approach were heavily influenced by bitcoin and, for this reason, are mainly interested in the economic nature of the coin, which was regarded either as commodity money or as fiat money, 
but always as digital money or digital (crypto)-currency. Works belonging to the second stream realise that the focus on the monetary nature of the coin is misplaced and a very narrow interpretation of the meaning of the coin and its value (Hayes, 2019). Starting with Ethereum, the coin becomes a digital token for the provision of services over the blockchain and, more generally, a legal claim (right) transacted over the network. As a result, the six distinct approaches focussing on the technology-symbolic interaction can be divided into two broad groups (of three approaches each) based on their understanding of the coin.

Three techno-economic approaches: One set of approaches mainly retains the interpretation of the coin as digital currency or money adopted by the political-economic approach to de/centralization, but extends and complements their intuitions to the technological dimension. For this reason, they can be labelled as techno-economic (or, sometimes, crypto-economic). Differently from the politicaleconomic approach, the three techno-economic approaches recognise that the coin's value and significance is not only affected by the political precepts (and institutional arrangements) shaping the interdependencies among social members off-chain, but also by the architectural and logical rules shaping the interdependencies among the nodes and elements of the network on-chain. In other words, the three techno-economic approaches recognise that the social value and significance of the coin depends on the specific logic and architecture adopted in order to transact, distribute and exchange the coin among the various nodes of the network on-chain.

Three techno-legal approaches: A second set of approaches departs more or less explicitly from the original understanding of the coin as an economic tool and treat it as a legal claim. These other three approaches, which we can call techno-legal, are mainly interested in the way in which the validity and the enforcement of a claim such as a tokenised property or a contract depends on the specific technological solution adopted. The techno-legal approaches all recognise that the meaning, validity and significance of a legal claim strongly depend on the architectural and logical rules shaping the interdependencies among the nodes and elements of the network on-chain. The shift from an economic to a more general legal understanding of the coin opens up new perspectives on the way in which de/centralization can be understood from a techno-legal perspective (and not merely techno-economic). These second set of techno-legal approach are sometimes also defined as institutional cryptoeconomics.

In sum, the techno-economic interpretation of de/centralization looks at how the technological characteristics of the blockchain network shape and affect the significance of the coin and the nature and origin of its value and vice versa. Decentralization is therefore defined in function of the changing network-coin relationship, while keeping the political and ethical dimension of the social community fixed.

The following three sub-sections will each discuss a techno-economic perspective with a fourth subsection summarizing the discussion: 1) protocol-logic: the decentralized protocol as a risk-free lender of last resort; 2) permission-logic: the decentralized protocol as a neutral validation mechanism; and 3) market-power: the decentralized network as an egalitarian distribution of capital;

\subsubsection{Protocol-Logic: Protocol as Risk-Free Lender of Last Resort}

The value and significance of a coin does not only depend on its interactions with the social dimension, but also on its interaction with the technology of the network. This is particularly evident in the case of digital metallism (the original understanding of cryptocurrencies such as bitcoin) 
where the coin's value is exclusively dependent on, and backed by, the network protocol and the onchain incentives generated by it, independently from the dynamics occurring in the socio-political dimension off-chain.

The bitcoin Proof-of-Work represents the original way to introduce economic incentives to ensure on-chain cooperation among all nodes and network integrity. The inherently costly and energy intensive process required for the solution of the cryptographic puzzle and the verification and validation of the transactions represents the way in which it was ensured that the coin could be perceived as an intrinsically valuable commodity by all those actors willing to participate in the process and therefore to incur in those costs. For this reason, the value of bitcoin was originally benchmarked to, and is still directly affected by, the costs to run the hardware performing the PoW algorithm.

The bitcoin PoW is usually defined a "decentralized" consensus protocol as it allows (in principle) any node to perform the verification and validation operations, while blockchains operating a "decentralized" consensus protocol are usually defined as "permissionless". However, the PoW is not the only example of "decentralized" consensus. Other consensus protocols ensure permissionless verification and validation of transactions, such as proof-of-stake (PoS), proof-ofauthority (PoA), and others (Narayanan et al., 2016: chapter 8). These alternatives become more and more appealing as some fundamental problems linked to the decentralized nature of the original PoW algorithm emerge, such as the: Extreme inefficiency and slowness; difficulty in scaling transactions; environmental impact and extreme energy consumption; tendency for on-chain concentration. Many blockchains are therefore abandoning PoW and are moving towards the adoption of PoS. Both are defined as "decentralized" and this creates some confusion on the meaning of decentralization in the case of consensus protocols, and on whether PoS is more or less centralized than PoW.

One way to distinguish the various protocols in function of their degree of de/centralization is to look at the nature of the interdependencies between the protocol and the coin that is univocally associated to the protocol run by the network. As discussed in section 5.2.1 above, the goal of many "decentralized protocols" is to disentangle the value of the coin from the social sphere, and to univocally link it to the technological dimension of the network. Based on that, here we define the protocol-logic approach as that specific subfield of the techno-economic interpretation of de/centralization that complements and extends the metallist interpretation of de/centralization, and defines de/centralization by looking at the way in which the value of the coin (intended as a digital commodity) is generated, set, and sustained in function of the interdependencies between the symbolic dimension of the coin and the technological dimension of the consensus protocol.

Consistent with a metallist approach to de/centralization, a network is more or less centralized according to a protocol-logic approach depending on whether the value of the coin depends on the contingent state of the network and on the way in which the various nodes decide to participate in the solution of the consensus protocol, or whether the value of the coin is independent from these and only depends on predefined protocol rules.

In the protocol-logic case centralization and decentralization are both defined in the case of socalled permissionless networks in order to distinguish degrees of de/centralization among permissionless protocols (among so-called "decentralized" protocols). A decentralized network 
protocol in the protocol-logic case does not characterise a permissionless network as opposed to a permissioned one (this will be the task of the permission-logic approach to de/centralization), nor it distinguishes centralization from decentralization based on the degree of asymmetric capabilities between nodes in validating transactions, We will define this later in the market power approach to de/centralization).

As a result, a decentralized protocol is characterised by the fact that the intrinsic value of the digital commodity only depends on the pre-established rules of the consensus protocol and is completely independent from the decisions, roles and capabilities of the single nodes participating in the consensus protocol. This means that in the case of a decentralized system value is independent from the specific and contingent state of the network and the way in which the various nodes decide to cooperate or compete in order to solve the consensus protocol, but it is established ex-ante by the protocol rules. In the protocol-logic approach the value of the coin is always intended as an objective and predictable property of the protocol independently from the network nodes. The coin value in this case becomes an intrinsic characteristic of the protocol itself, consistent with the spirit of digital metallism. Stated differently, decentralization implies a technological value of the coin defined bydesign. On the other hand, centralization in the protocol-logic approach emerges whenever the technological value of the coin is not set ex-ante and it is not a predictable and predefined property of the network protocol, but can change and fluctuate based on the contingent state of the network and on the contingent decisions taken by the nodes participating in the consensus mechanism. In this case the coin value is not independent from the decisions of the single nodes and therefore cannot be understood as an intrinsic characteristic of the network protocol.

Given the distinction between centralization and decentralization in the protocol-logic approach, a PoS protocol in which the rewards to nodes "staking" their coins in the consensus process are set by the protocol rules independently from the contingent state of the network is more decentralized than a PoW consensus protocol where rewards to miners (in the form of fees and mining rewards) can change and adjust based on the contingent state of the network and on the contingent decisions and conditions of the various nodes. This does not mean that a PoS consensus protocol is necessarily more decentralized thank a PoW consensus protocol, it only means that this is true limited to the protocol-logic definition of de/centralization. Alternatively, a peculiar PoW where all mining nodes only get a pre-established reward for their service based on the predictable amount of energy consumed in the process will be as decentralized as a PoS protocol.

The protocol-logic approach to define de/centralization has various similarities to the metallist approach. Both define decentralization as independence, rather than in terms of symmetric interdependence. In both the value of a coin does not depend on the specific interactions and organization of the elements of a system but only on pre-established rules that are immutable and independent from (thus exogenous to) the elements themselves.

Both perspectives, furthermore, intend decentralization by looking at the extent to which the value of the coin is independent from the contingent ebbs and flows of the system considered (either social or technological). In both cases the coin value becomes an exogenous and risk-free benchmark of value within the system in cases of decentralization. Centralization, on the other hand, denotes a situation where the coin value endogenously depends on the state of the system, and is therefore endowed with some risk and, consequently, with some variable and unpredictable return dependent 
on the specific interdependencies of the system actors (metallist) or network nodes (protocol-logic). While the coin in a decentralized PoW has the characteristic of an intrinsically valuable safe-haven asset (it behaves like a digital store of value), some authors are noticing that the coin in a decentralized PoS behaves more like a risk-free government bond, or a (risk-free) central bank base rate (Roda, 2019).

In conclusion, what characterises decentralization in the metallist and protocol-logic case is precisely the fact that the network is able to act as a lender of last resort and to guarantee a risk-free base rate on the coin, without the need for any central institutions managing it and baking it. This makes the value of the coin independent from the potential asymmetries of the system (social and technological). This characteristic of decentralized PoS has triggered new ways to benefit from the coin's risk-free "intrinsic" returns, notably the so-called "stake-as-a-service", which however looks dangerously similar to private banking (Casey, 2019). This is a plastic example of the fact that the approach adopted by the protocol-logic approach (as for the metallist approach) can characterise de/centralization according to one specific understanding of the coin-network interaction. A system decentralized according to the protocol-logic approach might not necessarily be decentralized according to other perspectives of the techno-economic interpretation, to which we now turn.

\subsubsection{Permission-Logic: Protocol as a Neutral Validation Mechanism}

While the protocol-logic approach is the equivalent of the metallist approach for the technological dimension, the permission-logic approach is the equivalent of the informational approach applied to the coin-network relationship on-chain. The first two define de/centralization by looking at the ways in which the coin can acquire social significance and social value independently from the presence of special actors endowed with asymmetric privileges, powers or rights to control, back and redeem the coin. The metallist and the protocol-logic define de/centralization by looking at the origin of the coin's value (off-chain and on-chain respectively) and conclude that decentralization characterises a condition of independence of the symbolic dimension from the underlying social (metallist) or technological (protocol-logic) dimension. This independence implies that the coin must be understood as a digital object having some form of intrinsic, fundamental, risk-free value that precedes and remains independent from, the social interdependencies among the members of the community (metallist) and/or the technological interdependencies among the network elements (protocol-logic). Value can be subjective and have an a-social origin.

Both the informational and the permission-logic approaches understand decentralization in terms of second-order symmetric interdependencies, rather than in the terms of independence. They are not interested in explaining where the coin value comes from, and both approaches assume that the coin can be intended as fiat money endowed with some social value. Differently from the protocollogic and the metallist approach, the permission-logic (and informational) approach assumes that the coin has a certain floating value that is determined by the contingent state of the networks (of social community) and can fluctuate and change in response to the decisions taken by the nodes of the network (or members of the social community). De/centralization is defined by looking at the asymmetries in the symbolic meaning and value of the coins at any given point in time emerging from the discrimination of the various members of the social community (informational approach), or elements of the network (permission-logic approach). 
In both approaches, a coin is decentralized whenever it is endowed with the same social value and significance independently from the identity of the actor transacting it (fungibility), and this makes it impossible to discriminate among its owners (neutrality). A coin is decentralized whenever its value and significance for anyone possessing it only depends on the collective and uncoordinated decisions of the various members, and does not depend on the unilateral decisions and identity of any specific actor.

There are two ways in which asymmetries here can be introduced in the symbolic dimension of the coin. Firstly, in the informational approach symbolic asymmetries can be used in order to generate social asymmetries by distinguishing one coin from the others and linking the value and significance of each coin to the specific identity of its holder. These symbolic asymmetries can be introduced in a token-based payment method, by simply verifying the nature of the coin and therefore ensuring that coin holders are favoured or penalised vis-à-vis the others. The informational approach is usually employed in order to discriminate and distinguish one member of the community form another one (Coin $\Leftrightarrow$ Social Community) and in the blockchain space is usually employed in the case of utility tokens whenever the subjective value and symbolic significance of a coin for a certain member must be different form the value that the same coin can have for another member: the social claims attached to a certain coin are subjective and asymmetric.

Secondly, symbolic asymmetries can also emerge as a result of architectural and logical asymmetries among the elements of the networks on-chain. In this case, the fungilibity and neutrality of a coin can be restricted by acting on the protocol of the blockchain: Centralization in the permission-logic approach denotes a situation where informational gatekeepers oversee transactions and can discriminate among nodes by verifying their status. These gatekeepers can decide which node can do what with its coin, whether a certain transaction should be authorised or not, and whether the social claim of a certain node is legitimate or not. Permission-logic centralization denotes a situation where the blockchain protocol allows for some special nodes endowed with special on-chain privileges or rights and having special on-chain capabilities. These are especially informational capabilities deriving from the fact that some nodes have a better understanding and overview of the state of the network and the status of the nodes than all other nodes.

Differently from the informational branch of the political-economic approach, the focus in this case is not on the asymmetry of powers among the members of the social community, but rather on the asymmetry of informational capabilities among the various nodes of the network. In this case a blockchain is decentralized if information can circulate freely and social coordination can be achieved without any need for a third party to reconcile private information and validate transactions. A centralized system is one where information capabilities are not evenly spread and social coordination among independent members can only be achieved if information has to be consolidated, reconciled and validated by a selected subset of nodes. Asymmetric information opens the door to asymmetric evaluations of the coin (lack of perfect fungibility) and therefore makes onchain discrimination among different nodes possible (lack of neutrality).

Informational asymmetries operate a token-based payment system where discrimination is based on the verification of the coin (its serial number, code and so on). Symbolic asymmetries are embedded in the coin and any node can verify the validity or legitimacy of the use of the coin. Conversely, the permission-logic approach operates an account-based payment system, where the legitimacy and 
validity of a transaction does not necessarily rely on the identification of the coin, but on the identification of the nodes undertaking the transaction. In this case centralization emerges as some special nodes operate as private banks, fulfilling the role of information intermediaries and brokers of information and value among coins on-chain. In this case, symbolic asymmetries depend on technological asymmetries of the network.

This type of centralized blockchains are usually called permissioned, while decentralized ones are usually called permission-less, and for this reason we named this specific techno-economic approach a permission-based interpretation of de/centralization. Technological asymmetries in a permissioned network are introduced de jure, by endowing different nodes with different rights. Depending on the specific protocol adopted and on the specific network, special nodes can be defined as full nodes, super nodes, mining nodes, master nodes, and so forth.

Both the protocol-logic and the permission-logic approaches share the intuition that the symbolic dimension is shaped by the nature of the protocol implemented and that the value and the significance of the coin might be affected by the different ways to operate the network. In both cases the degree of centralization of the network has a direct effect on the way in which the nature of the coin is perceived. Yet, the protocol-logic and the permission-logic interpretations of de/centralization might be incompatible. Both Proof-of-Stake (PoS) and permissioned logics where introduced to obviate to the shortcomings of the permission-less Proof-of-Work (PoW), but while the former is decentralized from a permission-logic point of view, the second introduces centralization.

A PoW protocol denotes a decentralized network under the permission-based interpretation, but it might generate more centralization from a protocol-based approach. The choice between the two might in turn depend on the way in which the coin is interpreted and on the political-economic approach adopted. A more centralized logic was favoured by those interpreting bitcoin as a store of value: centralization helped when it comes to the preservation of value of the coin deriving from an increased efficiency of the system. However, a decentralized logic of the infrastructure was mainly favoured by those interpreting the bitcoin in informational terms: as a means of exchange for interpersonal transmission of information.

Furthermore, different ways in which the information of the transaction is verified and validated by the network imply different expenditures of energy and, more generally, a different cost of running the system. The electricity cost sustained in order to validate transactions is a key variable entering the economic incentives of the validators in case of a permissioned PoW, but not necessarily under PoS. The value of the coin is not immune to the level of production cost that has to be sustained by the various validating nodes in order to operate the network. As a result, even when it comes to the direct expenditure sustained in order to operate the network, the degree of centralization of the network has a direct effect on the value of the coin.

None of these protocol solutions are immune to the concentration of power within fewer and fewer nodes than expected, which constitutes a third type of techno-economic centralization, marketpower, to which we now turn. 


\subsubsection{Market-Power: Network as an Egalitarian Distribution of Capital}

The previous sub-sections discussed the protocol-logic and the permission-logic. The former's interpretation of de/centralization considered at how the nature and significance of a coin is shaped by different protocol solutions, such as the shift from PoW to PoS, i.e., how the objective value and significance of the coin and its interdependencies with other coins are affected by asymmetries in the protocol logic. The latter's interpretation considers how the subjective value and significance of the same coin for different nodes is shaped by different consensus protocols based on the different role and status of these nodes within the network: how asymmetries in the subjective values and significances of a coin among different nodes transacting it are affected by asymmetries in the protocol logic.

Both consider how a/symmetries codified in the protocol logic of the technological dimension generate a/symmetries in the symbolic dimension. These asymmetries in the technological dimension might, in turn, themselves be the outcome of more fundamental asymmetries in the social dimension, which will be the topic of the three techno-political interpretations discussed in the three techno-legal interpretations discussed further below in this document. However, neither the protocol-logic approach nor the permission-logic approach considers the possible reverse causality between the two dimensions, namely the fact that asymmetries in the symbolic dimension might trigger asymmetries among the nodes of the network in the technological dimension. This is the domain of the third techno-economic interpretation of de/centralization, which we call the market power interpretation.

As opposed to the two previous interpretations, this approach seeks to understand interactions between the value of the coin and the economic decisions taken by the nodes. This stream of work belongs to the economic literature and is mainly interested in applying mechanism design and the theory of incentives to understand how the degree of centralization over the blockchain is endogenous shaped by actors' incentives. The incentives are first and foremost the coins that the validators can obtain as rewards for their roles of gatekeepers of the blockchain system through a consensus process. This approach then sees the coin as a pure economic instrument endowed with social value in exchange adopted as a reward for the work and effort spent by the various nodes. This interpretation of the coin as income, or reward for labour, is consistent with both the metallist approach (the coin's value has its origin the costly validation process) and the informational approach (the coin used as a fungible means of exchanged used to reward labour). In either case, what interests the market-power approach is the economic value of the coin not its legal significance and for this reason this is clearly a techno-economic approach, rather than a techno-legal one.

The market-power approach to de/centralization is now also referred to as "cryptoeconomics". ${ }^{38}$ Actors are utility maximises and profit seekers. The goal of this new field of the economic literature is the design of the optimal protocol that can maximize the welfare of the system while also guaranteeing on-chain "decentralization" among its actors. Consistent with a techno-economic approach, the focus of this type of analysis is the way in which the specific architecture and logic of

38 For an overview of "cryptoeconomics", see the useful summaries provided by Consensys (https://consensys.net/academy/blockchain-basics-book/cryptoeconomics); the Ethereum foundation (https://cryptoeconomics.study/); and Binance (https://www.binance.vision/economics/a-beginnersintroduction-to-cryptoeconomics) among others. 
the network infrastructure interact with the chosen values of the monetary rewards - typically, the coin, but some other times even the level of transaction fees. Some cryptoeconomics models are dynamic, meaning that on-chain concentration is a function of the incentives to enter (access) a blockchain system, others are static, in the sense that the competitive dynamics occurring on-chain only depend on a fixed number of actors operating over the blockchain. A non-comprehensive list of the works belonging to this stream is represented by: Abadi and Brunnermeier (2018), Biais et al (2019), Bonneau (2018), Bohme et al (2015), Budish (2018); Chiu and Koeppl (2017), Cong and He (2019), Easley et al. (2019), Huberman et al. (2017), Kroll et al. (2013), Ma et al. (2018). Narayanan and colleagues (2016: 130-136 \& 190-206) provide an exhaustive overview of the economic and technological problems linked to the incentives embedded in any crypto consensus algorithm.

As a result, de/centralization in the cryptoeconomic case can be defined as market power and market structure in economics and industrial organization is usually defined as follows. A technoeconomic system is decentralized if no node has the special power to unilaterally and purposefully affect the value of the coin independently form others. This approach can be understood as a conflation of the previous two techno-economic approaches. Similar to the metallist and the protocol-logic interpretations, a system is understood as decentralized whenever the social value and significance of the coin results from the uncoordinated and collective decisions of the various nodes, and centralized whenever it rests on the asymmetric powers of certain nodes, whose decisions count more than those of other nodes. Similar to the informational and permission-logic approaches, centralization reflect asymmetries in the status, capabilities and valuations of the coin among the different nodes (and not pre-defined and objective rules codified ex-ante in the blockchain protocol and homogeneously valid across all nodes indifferently).

The market power approach does not go as far as the other two approaches in wondering what confers economic value to the coin, nor does it consider the fact that the nature and significance of the coin depends of the specific social and technological context. But differently from the previous two interpretations, the market power approach introduces the new possibility that the blockchain protocol and the coin value do not necessarily interact directly, but that the interdependencies between the two might be mediated by the economic decisions of the various nodes that operate in response of a given value, and given certain protocol rules. The nodes in the market-power approach act as key intermediaries between the symbolic and the protocol dimension.

The field of cryptoeconomics is interested in devising the optimal protocol knowing that a change in protocol not only change the value of the coin, but in turn also provides a different set of incentives to the nodes whose decisions endogenously affect the value of the coin. The decisions of the nodes concern the economically optimal level of investments in those assets that allow them to gain coins as rewards for their participation in the consensus mechanism codified in the blockchain protocol. These investments can either be in specialised mining assets (in the case of PoW) or in voting rights (in the case of PoS). As a result, the market-power approach recognises that the distribution of power among the nodes is not given but reflect a Sutton-type strategic game (Sutton, 1991; 1997) where nodes escalate their investments and, in so doing, concentrate the power to validate and approve transactions in fewer and fewer hands. In economics terms, cryptoeconomics studies a situation of endogenous investment levels leading to an endogenous (market) "structure" of the blockchain network. 
Centralization in the market-power approach reflects a situation of on-chain concentration of capital in few hands, thus generating on-chain power asymmetries among nodes. Gencer and colleagues (2018) provide one of the first empirical studies comparing the degree of decentralization of bitcoin and Ethereum networks along this line, by checking the concentration (or dispersion) of mining power among bitcoin and Ethereum miners. Obviously (and this is a core aspect of the marketpower approach), the incentives to escalate in capital investment is directly affected by the social value and significance of the coin in turn, making concentration more likely the higher the return on the investment and therefore the more valuable the coin paid as a reward. While the protocol, and permission-approaches take the strategic decisions of the nodes as given, the market-power approach takes the de/centralized nature of the currency (and the origin of its value) as given. For this reason, the market-power approach complements and extends the other techno-economic approaches to decentralization, it does not replace these perspectives. At the same time, an excessive centralization of the validation power among the nodes of the chain might negatively impact the value of the coin in the eyes of the members of the social community, thus in turn shifting the value and appeal of the coin.

In the light of these double endogeneities, it is possible to summarise the meaning of de/centralization in the market-power approach as follows. Decentralization defines a situation of symmetric interdependencies between the coin's value and significance and the structure of the network in terms of distribution of validation power among the nodes. This means that a decentralized network is one where no node (or subset of nodes) has the power to exercise control over the coin and to univocally determine its value and significance of a coin through its escalation in validation investments. This also means that the distribution of the validation power among the network's nodes does not reflect the effects on the coin's value and significance of the decisions of any specific node on above the others. This situation of symmetric interdependencies retraces the nature of pre-ASIC or ASIC-resistant blockchains, where the technological and economic viability to escalate in CPUs or GPUs investments that concentrate mining equipment in few hands is particularly reduced. In this case, the value and social significance of a coin depends on the solution of the cryptographic protocol by the part of the network's nodes in the same way in which each node sees the value of the coin as a given, thus acting as an incentive-taker, rather than as a valuesetter.

At the same time, decentralization can also denote a situation where the incentives perceived by the nodes to verify and validate transactions and claims are independent from their escalation in investments, and therefore where the distribution of validation power among the nodes is independent from the value of the coin. This is the situation of a "democratic" PoS where each node as an equal amount of voting rights to validate transactions, and therefore an equal level of incentives, independently from their capital expenditures, and their relative status and the value of the coin itself. In this case market-power decentralization corresponds to a situation of protocollogic decentralization - independence between the symbolic and the technological structure of the network).

On the other hand, centralization emerge whenever there are asymmetric interdependencies between the value or significance of the coin and the distribution of the validation power among the nodes, meaning that asymmetries in the symbolic dimension foster asymmetries in the technological dimension and vice versa. This represents a situation where asymmetries in value among different 
coins generate incentives among the nodes of a certain network to invest in coin-specific assets that allow them to increase the rewards obtained in one coin at the expenses of the rewards obtainable in other coins. This generates escalation in investments and capital accumulation for some specific coins, and therefore asymmetric nodes in turn.

The current history of cryptocurrencies and cryptoassets shows that market-power centralization appears in both PoW and PoS. Both can be understood as two different types of capitalistic centralization, revealing a pure economic understanding of centralization (see section 2.4).

With respect to PoW, market-power centralization characterises the typical situation where the greater value and popularity of some coins compared to other alternative coins incentivizes nodes to shift from general purpose machines such as CPUs or GPUs towards specialised equipment, such as ASIC mining hardware. In this case, the economic justification of the investment derives from the greater value of the coin compared to others. At the same time, centralization also denotes the opposite causality. As fewer powerful nodes operating ASIC farms can now operate as value-setters, rather than value-takers, they can directly affect the value and significance of their coins vis-à-vis the same coins held by the other nodes of the network, and vis-à-vis other coins altogether. The first case of centralization reflects a so-called "majority attack" (or $51 \%$ attack) leading to the manipulation of the value and significance of certain specific coins vis-à-vis the others internally to a single blockchain network. The second case represents the essence of a market manipulation in the crypto-space, where the decisions taken by few powerful nodes of a blockchain network create unjustified and inflated valuations of a coin vis-à-vis other coins supported by other blockchains.

With respect to PoS, market power centralization emerges whenever the voting rights held by each node do not reflect a democratic principle (one-vote per head), but are weighted according to the economic relevance of the node: nodes with higher levels of coin stock or capital investment also obtain higher stakes, which make them more relevant than nodes with lower stakes. This system where on-chain voting rights are not counted but weighted is also usually defined in the cryptoliterature as "plutocracy" (Buterin, 2018). As a result, the interpretation of the coin as a digital commodity backed by protocol-logic decentralization also supports market-power decentralization, while an interpretation of the coin as digital fiat money might lead to on-chain capitalistic centralization (but do not need to).

\subsubsection{Summary of Techno-Economic Interpretations of De/centralization}

The three techno-economic interpretations of de/centralization discussed above conceive the coin as an economic tool endowed with some economic value that can be either monetary (in exchange) or subjective (in use) depending on the specific interpretation of the coin adopted. All three technoeconomic approaches reveal different reasons why the value and significance of a blockchain coin can be affected by asymmetries in the network technology and, vice versa, why certain asymmetries in the coins' values and significance might trigger on-chain asymmetries among the nodes of the network.

A blockchain network is decentralized from a protocol-logic perspective only if the objective value of the coin is completely independent from the contingent state of the network, interdependencies and decisions of the nodes. The value of the coin is common to all nodes and is predefined ex-ante by the protocol rules. This peculiar interpretation of decentralization derives from the fact that the protocol-logic approach adopts the metallist understanding of decentralization that implies that 
whenever the value of the coin depends on the contingent agreements, interactions and interdependencies between different actors or elements, some form of centralization will always emerge.

A blockchain network is decentralized from a permission-logic perspective whenever the value of the coin can change and fluctuate based on the contingent state of the network, but it is common to all nodes and does not depend on the asymmetries in special status between the nodes validating the transaction and the ones transacting. In this case the coin can be a socially constructed means of exchange endowed with pure exchange vale on-chain, but centralization emerges whenever the consensus process is permissioned.

A blockchain network is decentralized from a market-power perspective whenever the value of the coin for any node cannot depend on the strategic behaviour and investment decisions of the same node: any node must remain value-taker, and no node can purposefully act in order to discriminate its coins from the ones of others.

The protocol-logic approach makes sure that the blockchain protocol renders network asymmetries among nodes irrelevant, the permission-logic approach makes sure that the blockchain protocol rules them out ex-ante (de jure), while the market-power approach makes sure that the incentives built in the blockchain protocol prevents on-chain network asymmetries to emerge ex-post as well (de facto).

Both the permission-logic and the market power approach treat centralization in terms of discrimination and inequalities among nodes. But while the permission-logic approach is mainly interested in making sure that a node's coin is not negatively discriminated against the ones of special status nodes, the market-power approach mainly makes sure that a node cannot positively discriminate its own coins cis-a-vis the ones of other less powerful nodes.

Table 6.3 and 6.4 recap the key characteristics of the three techno-economic approach to de/centralization

\begin{tabular}{|l|l|}
\hline $\begin{array}{l}\text { Techno-Economic second- } \\
\text { order interdependencies }\end{array}$ & $\begin{array}{l}\text { How the technological characteristics of the blockchain network shape and affect the significance of the } \\
\text { coin and the nature and origin of its value and vice versa. }\end{array}$ \\
\hline \hline Approach & $\begin{array}{l}\text { Tries to understand how the value of the coin is endogenously shaped by the network technology and } \\
\text { vice versa: a change in the way in which the nodes can communicate and transact on-chain and can } \\
\text { verify, validate and append transactions directly affects the economic value and significance of the coin } \\
\text { and vice versa. }\end{array}$ \\
\hline $\begin{array}{l}\text { Definition of } \\
\text { de/centralization }\end{array}$ & $\begin{array}{l}\text { How asymmetries in the social significance and meaning of coin (the symbolic dimension of a digital } \\
\text { network) depend on the asymmetries among the elements of the network (the technological dimension) } \\
\text { and vice versa. }\end{array}$ \\
\hline $\begin{array}{l}\text { Unit of analysis } \\
\text { (De/Centralization applies } \\
\text { to) }\end{array}$ & The blockchain network (\& protocol) \\
\hline \hline
\end{tabular}

Table 6.3: Overview of the techno-economic perspective. 


\begin{tabular}{|c|c|c|c|}
\hline & Protocol-logic & Permission-Logic & Market-power \\
\hline & \multicolumn{3}{|c|}{ Decentralization } \\
\hline $\begin{array}{l}\text { Meaning of } \\
\text { decentralization }\end{array}$ & $\begin{array}{l}\text { Independence of the symbolic } \\
\text { dimension from the technological } \\
\text { dimension }\end{array}$ & $\begin{array}{l}\text { Symmetric interdependence } \\
\text { between the symbolic dimension } \\
\text { and the technological dimension }\end{array}$ & $\begin{array}{l}\text { Symmetric interdependencies or } \\
\text { independence between the symbolic } \\
\text { dimension and the technological } \\
\text { dimension }\end{array}$ \\
\hline $\begin{array}{l}\text { Condition for } \\
\text { decentralization }\end{array}$ & $\begin{array}{l}\text { Techno-symbolic Independence: } \\
\text { The intrinsic value of the digital } \\
\text { commodity only depends on the } \\
\text { pre-established rules of the } \\
\text { consensus protocol (ex-ante) and is } \\
\text { completely independent from the } \\
\text { specific and contingent state of the } \\
\text { network and the way in which the } \\
\text { various nodes decide to cooperate } \\
\text { or compete in order to solve the } \\
\text { consensus protocol }\end{array}$ & $\begin{array}{l}\text { Symbolic and technological } \\
\text { symmetries: } \\
\text { The value of the coin is not } \\
\text { predefined ex-ante and can } \\
\text { fluctuate according to the } \\
\text { contingent state of the network. } \\
\text { At any given time, identical coins } \\
\text { are endowed with identical value } \\
\text { (fungibility of the coin) and the } \\
\text { same coin gives identical claims } \\
\text { to any member (privacy and } \\
\text { equality) }\end{array}$ & $\begin{array}{l}\text { Symmetric interdependencies: No node } \\
\text { (or subset of nodes) has the power to } \\
\text { exercise control over the coin and to } \\
\text { univocally determine the value and } \\
\text { significance of a coin by escalating its } \\
\text { capital investments in validating assets. } \\
\text { Independence: } \\
\text { the incentives perceived by the nodes to } \\
\text { verify and validate transactions and } \\
\text { claims are independent from their } \\
\text { escalation in investments (distribution } \\
\text { of validation power among nodes) }\end{array}$ \\
\hline $\begin{array}{l}\text { Causality / } \\
\text { Directionality }\end{array}$ & Absent & Bidirectional / Symmetric & Bidirectional / Symmetric \\
\hline Nature of value & Objective and a-social & Objective and social & Objective and social \\
\hline \multirow[t]{2}{*}{$\begin{array}{l}\text { Implications for } \\
\text { the } \\
\text { technological } \\
\text { sphere }\end{array}$} & $\begin{array}{l}\text { Network protocol as a risk-free } \\
\text { lender of last resort }\end{array}$ & $\begin{array}{l}\text { No identification-based } \\
\text { payments (legal equality and } \\
\text { privacy of all nodes) }\end{array}$ & $\begin{array}{l}\text { Economic equality of all nodes (no } \\
\text { economic power) }\end{array}$ \\
\hline & \multicolumn{3}{|c|}{ Centralization } \\
\hline $\begin{array}{l}\text { Meaning of } \\
\text { centralization }\end{array}$ & $\begin{array}{l}\text { Interdependence between the } \\
\text { symbolic dimension and the } \\
\text { technological dimension }\end{array}$ & \begin{tabular}{|l|} 
Asymmetric interdependence \\
between the symbolic dimension \\
and the technological dimension
\end{tabular} & $\begin{array}{l}\text { Asymmetric interdependence between } \\
\text { the symbolic dimension and the } \\
\text { technological dimension }\end{array}$ \\
\hline $\begin{array}{l}\text { Condition for } \\
\text { centralization }\end{array}$ & $\begin{array}{l}\text { Coin value and significance depends } \\
\text { on the contingent on-chain } \\
\text { interdependencies among nodes of } \\
\text { the network and are defined ex- } \\
\text { post }\end{array}$ & \begin{tabular}{|l|} 
De jure symbolic and \\
technological asymmetries: \\
some special nodes have the \\
protocol right to determine the \\
validity and the value of other \\
nodes' coins leading to potential \\
asymmetries among identical \\
coins (no fungibility or neutrality \\
of the coin) and among nodes \\
(no privacy or equality) \\
\end{tabular} & $\begin{array}{l}\text { De facto symbolic and technological } \\
\text { asymmetries: some special nodes have } \\
\text { the economic power to determine the } \\
\text { validity and the value of other nodes' } \\
\text { coins leading to potential asymmetries } \\
\text { among identical coins (no fungibility or } \\
\text { neutrality of the coin) and among nodes } \\
\text { (no privacy or equality) - capitalistic } \\
\text { inequality }\end{array}$ \\
\hline $\begin{array}{l}\text { Causality / } \\
\text { Directionality }\end{array}$ & $\begin{array}{l}\text { From the technological to the } \\
\text { symbolic dimension }\end{array}$ & $\begin{array}{l}\text { From the technological to the } \\
\text { symbolic dimension }\end{array}$ & Bidirectional / Asymmetric \\
\hline Nature of value & Objective and social & Subjective and social & Subjective and social \\
\hline $\begin{array}{l}\text { Implications for } \\
\text { the } \\
\text { technological } \\
\text { sphere }\end{array}$ & $\begin{array}{l}\text { Presence of private traders and } \\
\text { brokers on-chain }\end{array}$ & $\begin{array}{l}\text { Presence of informational } \\
\text { intermediaries for the } \\
\text { settlement of transactions such } \\
\text { as private banks or on-chain } \\
\text { institutions entitled to regulate } \\
\text { the value and validity of the } \\
\text { coins on-chain }\end{array}$ & $\begin{array}{l}\text { Presence of private corporations or } \\
\text { private actors accumulating capital } \\
\text { endowed with economic power to } \\
\text { distort and shape the value and validity } \\
\text { of the coins on-chain }\end{array}$ \\
\hline
\end{tabular}

Table 6.4: Summary of the three techno-economic perspectives: the protocol-logic, permission-logic, and market-power

Having covered the three techno-economic perspectives on the second-order interdependencies between the Coin and Network Technology, the following three sub-sections discusses the three techno-legal perspectives on the same set of second-order independencies with a fourth subsection summarizing the discussion: 1 ) platform-logic: the decentralized network as a neutral and open general-purpose technology; 2) code-is-law: the decentralized network as an on-chain governance mechanism; and 2) principal-agent: the decentralized network as coordination mechanism for the on-chain division of labour. 


\subsubsection{Platform-Logic: Network as a Neutral and Open General-Purpose Technology}

The work discussed in this sub-section breaks away from the economic interpretation of the coin and treats the coin as a proper token or digital asset that can be used for different purposes and in different ways for the provision of a variety of services. The fundamental event in this respect was the launch of Ethereum in 2015 defined by its developer as a Touring-complete "Decentralized Application Platform" (Buterin (2014). The launch of Ethereum broadened the meaning of the coinnetwork relationship and the role of the blockchain protocol in mediating this relationship. The platform interpretation of de/centralization broadens the interpretation of the coin and treats it as a generic social claim. At the same time, it also broadens the concept of blockchain as a generalpurpose technology for the autonomous on-chain enforcement of rights, away from its initial understanding as a mere distributed ledger technology for the autonomous settlement of financial transactions. This change in the way in which the coin-network relationship is conceived is the essence of the techno-legal interpretation of de/centralization, as opposed to the techno-economic interpretation discussed above.

In order to understand the essence of the symbolic-technological interdependence (Coin $\Leftrightarrow$ Network Technology) in the platform logic, it is necessary to distinguish between two different types of coins. A native coin is defined as that token that is used by the consensus protocol in order to provide the optimal incentives in order to autonomously enforce transactions and settle claims over the blockchain. XRP and ether are two basic examples of a native coin. The value of native coins is univocally linked to the value of the protocol and can therefore be intended as digital commodities, in accordance with the metallist and the protocol-logic approach. However, the same network can also be used as a platform for the operation and exchange of other secondary coins for the provision of an array of alternative services over the top. We can define these as App-coins, or simply as tokens. The value of these App-coins is directly proportional to the utility and the value of the service that they provide.

The works belonging to the platform approach recognise that there is an inherent univocal relationship between the coins operated over a blockchain network and the protocol rules governing that network. Each coin has its own associated network protocol and can usually only be operated according to the protocol rules of that specific network. Yet, the presence of a multiplicity of concurrent App-coins might generate a competitive race among the various blockchain networks in order to gain the widest adoption among users. Because of network effects, the wider the adoption base the higher the chances that the underlying blockchain network will become valuable and widespread, and that the values of the coins associated with the platform (both native and App) will increase vis-à-vis the value of the coins of the other alternative blockchain networks. Coins in this case can be both native and cryptotokens operated over-the-top.

The problem faced by the platform-based approach is the determination of the optimal scope of applications and App-coins supported by the network that maximizes the adoption of the network and therefore the value of its native coin vis-à-vis other blockchain ecosystem. In other words, in this steam of literature blockchain networks are treated as platforms subject to network effects, and the concept of centralization is interpreted through the lenses of platform theory (Catalini and Gans, 2018; Gandal and Halaburda, 2016; Gans and Halaburda, 2015, Polasik et al., 2016; Luther, 2016). For this reason, and consistent with the literature on the topic, we define this interpretation of de/centralization as the platform approach. 
Consistent with the approach adopted by platform studies in management and organization studies, the platform approach interprets de/centralization in the light of the typical tension between the creation of value fostered by platforms open to a variety of services and functionalities, and the capture of value ensured by the closed and proprietary nature of the coins allowed to operate over the network (Benlian et al., 2015; Boudreau, 2010 and 2012; Eisenmann et al., 2009; Gawer and Henderson 2007; West, 2003). The fundamental problem analysed by this peculiar approach of the coin-network relationship is whether it is better for the success and wide adoption of the network to open the platform to other "non-native" coins by implementing agnostic or interoperable protocols, or if it is better to discriminate and deny access to other coins by remaining closed and autarkic. In other words, it is a dilemma of whether it is convenient to: 1 ) transform the blockchain network into a platform able to operate a multiplicity of non-native (non-proprietary) coins so to exploit a proliferation of use cases and gain increasing utility and value in the eyes of the users (value creation), or 2) to maximise value capture by remaining closed and forcing all members of the social community to exclusively deal with the proprietary coins provided by the platform. This approach adopts the typical normative paradigm of the efficiency or welfare analysis of the managerial and economic literature.

In this case de/centralization can be understood according to the categories of openness and neutrality. A network is centralized whenever it is not neutral with respect to the coins that can be operated over it and whenever it discriminates among various compliant coins or deny access to them altogether. On the other hand, a decentralized network is one characterised by symmetries among the various coins given a certain protocol, in the sense that all protocol-compliant coins are free to operate and being transacted over the network. The political requirements of equality and liberty characterising decentralization in this case apply to the coins operated over-the-top rather than the members of the social community, or to the nodes of the network: a decentralized network is open to support any protocol-compliant coin (liberty of all coins implies open access) and does not discriminate among them (equality of all coins implies neutrality). Stated differently, a centralized network behaves like a proprietary and/or closed platform, while a decentralized one can be intended as a neutral and open one.

The platform approach focusses on the way in which the value of the (native) coin is endogenously linked to, and affect by, the technological choices concerning the openness and neutrality of the underlying network. Different technological decisions on the openness and neutrality of the network have implications on the value of the native coin. The platform approach does not interpret de/centralization in terms of de facto market power, or de jure rights. De/centralization in this case is most appropriately defined in function of the concept of control, consistent with the platform literature (Boudreau, 2010; Eaton et al, 2015; de Reuver et al, 2018). A centralized technology represents a closed and/or non-neutral network that retains complete central control over the set of App-coins that can be operated over its protocol. Conversely, decentralization in this case denotes a situation where the control over the set of App-coins that can be created, launched, transacted and operated over the infrastructure is left to third parties among the members of the social community.

The paramount case captured by this type of analysis is the distinction between the Bitcoin infrastructure and the Ethereum platform. Both bitcoin and ether are univocally linked to their blockchain protocol and, for this reason, they derive their value from the popularity and trust in their respective blockchain networks - from a metallist point of view they can both be understood as 
digital commodities only backed by the technology. However, Bitcoin used to be characterized by a rigid protocol that does not allow any other coin to be transacted but for its native one. Ethereum has adopted a general-purpose Turing-complete protocol, ${ }^{39}$ that is general purpose and flexible enough to allow for a wide array of actors to create, launch and transact their own private coins, provided they are ERC-20 (or ERC721) compatible. Another very relevant open issue, still belonging to this interpretative approach is the problem of inter-network interconnection and interoperability. Networks can become platform not only because they decide to relinquish control to the edges of the network, but also because they enable the interconnection of different actors belonging to heterogeneous system. In this case, centralized exchanges (CEX) are the archetypes of centralization of control, while decentralized exchanges (DEX) represent preliminary attempts to behave like platforms with no control over the interconnection process. Even in this case, the centralizationdecentralization dichotomy reflects the problem of harmonizing different functionalities and Appcoins under the same protocol rules.

\subsubsection{Code-is-Law: Network as On-chain Governance Mechanism}

The second stream of the techno-legal approach is probably one of the most famous and acknowledged understandings of de/centralization. The code-is-law approach mainly emerged in the legal literature and deals with the role of code in shaping the decentralized on-chain governance of the blockchain. Many of these works draw on Lessing's (1999) argument that in the cyberspace "code is law" and use it in order to understand the governance role of smart contracts for governing Decentralized and Autonomous Organizations (DAOs) - here used as a generic concept and not to denote the specific case of the DAO investment fund.

Differently form the protocol-logic approach of de/centralization the code-is-law approach is not interested in defining de/centralization in function of the nature and significance of the coin and the origin of its value. Differently from the permission-logic approach, the code-is-law approach does not define de/centralization based on the different on-chain rights of nodes to supervise, check and validate the legitimacy of the transactions of coins between anonymous (pseudononimous) members (in other words, to act as informational gatekeepers). Moreover, differently from the market-power approach the code-is-law approach is not interested in fine tuning the right incentives in order to avoid ex-post accumulation of capital and power in the hands of few nodes. Conversely, the code-is-law approach developed following the implementation of smart contracts on the Ethereum platform.

$\mathrm{De} /$ centralization in this case is defined by looking at the way in which contractual conditions are observed and verified and claims are subsequently autonomously enforced following the verification of certain contextual conditions. The protocol, in this case, absolves the role of a legal institution regulating and enforcing contractual conditions among contracting parties. For this reason, the blockchain technology (and its protocol) in the code-is-law approach can be regarded as an on-chain governance mechanism where the conditions for the execution and enforcement of a contract are

\footnotetext{
${ }^{39}$ There are a range of ways in which Turing-completeness can be characterized, but easiest explained in terms of procedural programming languages, then to be Turing equivalent, such language must contain versions of the three basic programming constructs allowing for sequences of statements, selection (e.g., if-then-else), and iteration (e.g., while-do loops). For a more precise and programming-language invariant description, see: https://en.wikipedia.org/wiki/Turing_completeness
} 
detailed ex-ante, and verified ex-post. The works in this stream usually focus on problems emerging from asymmetries in information between two contracting parties with respect to terms and contextual conditions and understand blockchain as a solution to this information asymmetry problem.

Thus, Atzori (2015) notes how the decentralized governance of the blockchain can potentially overcome the state in the enforcement of individual rights and replace it with a set of bilateral private relationship, Rejers and colleagues (2016) study blockchain sovereignty in terms of social contract theories, Wright and De Filippi (2015) identify a new subset of law, defined as lex cryptographia, which would substitute standard legal rules in the admiration of DAOs through smart contracts (see also De Filippi and Wright, 2018, chapter 4). Other works highlight problems and limitations of the decentralized cryptographic mechanisms for the enforcement of individual claims and rights (Sklaroff, 2017; Verstraete, 2018; Werbach, 2018; Werbach and Cornell, 2017).

All these works are joined by their common focus on the way in which the transaction and exchange of valuable claims can be guaranteed, verified and automatically enforced by the operation of a "decentralized" governance mechanism over the blockchain infrastructure. Decentralization in this case qualifies the nature of the blockchain protocol, not the nature of the coin, or the origin of its value. In the code-is-law approach decentralization is mainly intended as the absence of an authority having legal power (residual rights) to verify facts, adjudicate between contracting nodes and to decide ex-post the course of actions to be taken based on its own understanding of the situation and personal considerations.

As a result, the difference between centralization and decentralization in the code-is-law approach retraces the distinction between the two adopted by the protocol-logic approach: they both define de/centralization based on the temporal distinction between ex-ante and ex-post decisions. But while the protocol-logic approach applies this difference to characterise the nature of the coin and the origin of its value (it is a techno-economic), the code-is-law approach applies the ex-ante/ex-post difference to distinguish between ex-ante verification and ex-post adjudication of a contractual relationship (it is a techno-legal interpretation). In both cases decentralization implies an ex-ante origin of the significance and value of the coin, which are independent from the peculiar contingencies and state of the network.

The two perspectives also share the same interpretation of the meaning of a centralized network, intended as a network where there is a special on-chain entity that operates as a backer of last resort (techno-economic) or as a ruler or adjudicator of last resort (techno-legal). The parallelism between the two approaches can be pushed even further. While the coin in the techno-economic case represents a risk-free investment guaranteeing a predictable base return backed by the rules of the protocol (intended as a trusted risk-free minter), the coin in the techno-legal case represents an uncertainty-free legal agreement that guarantees a predictable "base protection" backed by the protocol (intended as a trusted and uncertainty-free enforcer).

The way in which asymmetries are understood in the case of centralization is however probably more similar to the interpretation given by the permission-logic approach. Technological asymmetries in the code-is-law approach might emerge from two reasons: asymmetries in the verification and observation of facts (informational asymmetries) and asymmetries in the interpretation, validation and adjudication of claims based on these facts (decisional asymmetries). 
In a decentralized network the terms and conditions on which claims are based should not be situated (architectural aspect) and not subject to the interpretation of certain specific nodes (logical aspect). This means that in a decentralized network not only can all contracts be enforced ex-ante and on-chain, but also that the information and facts on which contractual terms are based should be common and identically perceived by all nodes on-chain. A decentralized network is, therefore, a network where all nodes can operate as distributed oracles, and can independently verify and check the contextual conditions.

Conversely, a centralized network is one where technological asymmetries among the nodes of the network generate symbolic asymmetries among the claims of the members. Symbolic asymmetries in this case denote a situation where similar claims held by different members lead to different outcomes based on the specific contingencies perceived by the nodes and/or the specific decisions and interpretations of the adjudicating arbitrator. Contingent-based enforcement and validity of claims implies subjectivity in the value and significance of a coin and might lead to discrimination among members - asymmetries breaching the equality and privacy conditions. In sum, centralization is mainly understood in terms of the subjectivities emerging from asymmetries in information and knowledge. In this case the blockchain is mainly an informational technology and, as such, it also fulfils the role of an institutional technology.

The code-is-law approach is heavily rooted in the economic theory of incentives based on incomplete contracts, (Tirole, 1999 and Maskin and Tirole, 1999), in the Transaction Cost Economics (TCE) literature (Williamson, 1979; 1985) and in the Coasean property right theory literature (Coase, 1960), and it represents the essence of institutional cryptoeconomics (Davidson et al., 2018; Arrunada and Garicano, 2018). All these works focus on the role of time and understand governance in the light of the incentives emerging from the ex-post adaptation of ex-ante incomplete contracts. As a result, in this case the word "governance" has its roots in the institutional economic literature and in the literature on the theory of the firm (Williamson, 1979; 1985; 1996; 1998). However, in the techno-legal approach adopted by the code-is-law interpretation governance remains on-chain and ex-post adaptation off-chain is ruled out.

\subsubsection{Principal-Agent: Network as Coordination Mechanism for On-Chain Division of Labour}

The last interpretation of de/centralization, the principle-agent interpretation, is strictly linked to the previous one and is still part of the so-called institutional cryptoeconomics. Both are interested in the way in which the blockchain network becomes a new technology for the organization and coordination of transactions among the various members of a social community. But differently from the previous code-is-law approach, the focus is not on blockchain as an autonomous enforcer of contracts, such as a court of justice, but on blockchain as a new organizational form for the alignment of incentives and the coordination of activities, alternative to markets and hierarchies. Although incentive asymmetries are still very relevant, the focus is mainly on the role of information and capabilities. Blockchain in this case is not understood as a tool for the unequivocal (uncertaintyfree) settlement and verification of the right version of facts or the correct state of the network.

In the principal-agent interpretation of de/centralization the blockchain network becomes a new technology for the organization and coordination of transactions among the various members of a social community. The focus is mainly on the organization of activities and tasks on-chain and on the 
emergence of private governance arrangements associated to the well-known make-or-buy problem (or division of labour).

The works in this area are numerous and not necessarily coherent. This might derive from the fact that different theories of the firm understand the make-or-buy problem differently. For instance, TCE is a theory of incentives based on incomplete contracts, as the works of Tirole (1999) and Maskin and Tirole (1999) are, but other theories of the firm based on incentives such as Grossman and Hart (1986) are not. At the same time, Coase's (1937) theory is mainly a theory of the firm based on market failures that is partially a theory of incomplete contract but it also contains some reference to incentives in the line of resource- and capabilities-based theories of the firm (Jacobidies and Winter, 2005). Another very relevant stream of the economic literature on the theory of the firm is the principal-agent theory (Holmstrom and Milgrom. 1991; Jensen and Meckling, 1976). Incentives-based theories of vertical integration that are also incomplete-contract theories of the firm (such as; Williamson, 1985) substantially overlap with the code-is-law approach as they treat governance arrangements in the same manner as arbitrators of last resort. Yet, there are many theories of the firm that do not inquire the vertical integration problem adopting an incomplete contract perspective (Gibbons, 2003).

In any case, all works that can be grouped under the "principal-agent" approach highlight how the asymmetry in information, incentives, and capabilities between two contracting parties generate all sorts of coordination failures, and therefore of transaction costs, for example due to opportunism, moral hazard, principal-agent, adverse selection, and so on. This can lead to costly intermediation and inefficient division of labour. Institutions in this case become tools to restore coordination and ensure efficiency among parties that would not otherwise naturally cooperate and agree on the best course of collective action to be taken. Under this perspective, blockchain becomes a new institutional arrangement alternative to hierarchies and markets (with the addition maybe of networks: Powell, 1990; Smith-Doerr and Powell, 2005 - see also Ziolkowski et al 2018a and 2018b).

The centralization-decentralization dichotomy should therefore be interpreted along these lines, by checking the distribution of decision rights among the various nodes of the network. Decision rights can either be intended as control rights or as property rights, depending on whether ownership and control are severed or not. Markets are decentralized as the locus of decision making is left to the single actors behaving in their self-interests. However, different actors might have different expertise, incentives, knowledge and opinions on a certain course of action. Centralized hierarchies are seen by the managerial and economic literature as central points of decision making emerging to reduce the divergence in incentives characterising different nodes of the system.

In contrast to standard hierarchies and market solutions, the blockchain becomes a powerful institutional arrangement to ensure the coordination of economic activities and collective production without any need for a central hierarchy, similarly to all distributed infrastructures (Benkler, 2006). Notable works that have adopted this interpretation and focussed on the on-chain governance of a blockchain are (among many others) by: Arrunada and Garicano (2018), Davidson et al (2016), Davidson et al (2018), Meunier and Zhao-Meunier (2019), Miscione et al (2018a and 2018b), Zachariadis et al. 2019, Ziolkowski et al. (2018). 
Based on our definition of a techno-legal approach, it is possible to define the principal-agent interpretation of de/centralization by considering the locus of decision rights in the context of the division of labour.

A centralized network is one where some nodes have the right to decide on behalf of other nodes, and thus exercise control over other nodes' activities, decisions or properties, i.e. the storage of their cryptographic keys, the operation of their mining hardware, the employment of their coins, and so forth. This is, after all, the essence of a hierarchy. Hierarchies are centralized as they aggregate the control of various assets and activities under the same decision maker. Hierarchies decouple decision and control rights from the title (claim) holder and makes the latter dependent on the decisions of the former. A decentralized network is one where each node takes all decisions concerning its own titles of property and claims "by itself", merely based on its own capabilities, information and knowledge and do not rely on others' capabilities, information, and knowledge. In the principal-agent approach a decentralized network is a network where there is no need to outsource tasks and activities to specialised nodes as all activities and capabilities are symmetrically distributed to all nodes so that all nodes can undertake all activities that are necessary to operate "by itself".

In the centralized case, technological asymmetries in the capabilities of the nodes generate subjective asymmetries in the way in which the coin is understood and valued. These are the asymmetries discussed in the permission-logic approach above. The essence of the principal-agent approach derives from the realization that these asymmetries might in turn generate a shift in the locus of decision rights from the asset owner (principal node) to the asset manager (agent node). The latter then becomes a central intermediary that acts on behalf of other nodes. The reverse is also possible: some nodes can decide to outsource the decisions concerning the employment of their coin to other third parties as a response to increasing asymmetries in the symbolic dimension as some nodes might be more specialised than others. In the case of a centralized network, asymmetries in the capabilities of the nodes lead to a specialization of the tasks of the various nodes and therefore to a division of labour.

On the other hand, decentralization emerges under perfect distribution of resources and capabilities and therefore under pervasive adoption of the "make" option vis-à-vis the "buy" option. In this case, symmetry in the symbolic dimension implies alignment of incentives and capabilities, meaning that there is no need to outsource tasks at all as nodes perceive the coin in the same way, and they would all follow the same course of action anyway. Thus, symbolic symmetry necessarily implies technological symmetry and, as a result, decentralized governance forms. In this decentralized case, each node retains control over its property (coins) and decides how to employ and use it. The blockchain intended as a distributed infrastructure adopts this understanding of decentralization.

It should be noted that the way in which the term "control" is employed in this case differs from the way in which "control" should be intended in the platform approach. In the case of platforms, control refers to the right (of the platform owner) to, broadly speaking, decide the rules of the platform. Control is about access to the platform and the way third parties ought to use and operate the platform, to be intended as the property of the platform owner. In the case of hierarchies, the logic is reversed: control refers to the right of the right holder to decide what others ought to do with their own property in virtue of some asymmetry between the two parties. Control in this case is 
not about exclusion of undesired hosts (as in the case of platforms); it is about command over others' decisions. The specialization of certain nodes into specific activities based on their capabilities and roles denotes some form of centralization based on some principal-agent (hierarchical) relationship. The current blockchain ecosystem has emerged as some nodes interconnected to the network specialised into certain activities of the blockchain "supply chain", leading to the outsourcing of control and decision rights over the coins. Some notable examples are: the emergence of wallet providers acting as third-party custodians of private keys; and the emergence of exchanges acting as specialised investors on behalf of third parties. The same holds for the emergence of mining nodes specialised in mining and validation of transactions, which are usually associated to broader mining pools and virtual pools directed by specialised third parties. Some nodes, usually called master nodes or super nodes, specialise in certain relay or transmission activities within the network for the broadcast of all transactions to all nodes. All these examples show an increasing degree of asymmetric interdependence of one node on other specialised service-providers within the network. More generally, any blockchain where light-nodes are distinguished from full nodes, manifest some degree of division of labour and specialization, and therefore some degree of centralization.

To summarize, decentralization in the principal-agent approach emerges whenever different nodes can operate independently under the condition of symmetric information, opportunities and capabilities. Mutual interdependence among nodes denotes a situation of asymmetric information, opportunities and capabilities. As a result, this approach characterises the relationship between the division of labour and decentralization in the opposite way in which it is understood by the standard theory of the firm.

Differently from the standard ideal market solution characterised by an extreme division of labour, decentralization in the case of blockchain is characterised by minimal (inexistent) division of labour, meaning that a decentralized network is one where all nodes are endowed with the same set of symmetric capabilities and, as a result, do everything in-house. The "make" option in this case implies the mining, verification, transmission, key storage, validation, voting, activities and so forth). In other words, according to the principal-agent interpretation of de/centralization, a perfectly decentralized blockchain is a blockchain exclusively operated by full nodes. On the other hand, differently from the standard hierarchical solution, the aggregation of different activities under the same "full" node is not understood as centralized response to a market failure (transaction cost) nor does it characterize a hierarchical solution, but as a precondition of symmetric distribution of information, capabilities and opportunities.

As a result, while hierarchies under the standard transaction-cost logic of Williamson (1985) and Coase (1937) reduce the extent to which labour can be divided among independent actors and emerge whenever the "make" option is more convenient and "economically rational" than the "buy" option, the exact opposite is true in the case of a centralized blockchain: centralization in the principal-agent perspective characterises a situation of greater division of labour and specialization of the tasks, where specialised nodes are mutually dependent on the actions and decisions of each other's. Consistent with the liberal understanding of liberty and equality, and differently from the neoclassical one, decentralization in this case corresponds to a situation where the absence of transaction costs leads to a minimal division of labour among the nodes on-chain (independence). The neoclassical understanding of decentralization is precisely the opposite: the perfect 
decentralization emerging from the ideal world of zero transaction costs lead to the maximum division of labour and interdependencies among economic actors.

One potential explanation of the reason why this paradox emerges derives from the peculiar way in which transaction costs are minimised in the blockchain: thorough the sharing and common access to the same common ledger and timestamp generating logical centralization of the distributed system. As a result, it is possible to conclude that while the nature of the interdependencies among the nodes can be treated as decentralized, the blockchain itself (as any logically centralized distributed system) is a sort of centralized super-hierarchy.

\subsubsection{Summary of Techno-Legal Interpretations of De/centralization}

The techno-legal interpretations of de/centralization recognize that the coin is a legal claim and that the technological dimension of the network has a direct impact on the way in which the various legal claims are treated and understood by the various nodes.

The platform-logic interpretation recognises that the range of applications and services transacted over a network can have an impact on the way in which the native coin of the network is valued and understood. A network can be more or less centralized based on its degree of openness to, and neutrality for, other App-coins. This is often the interpretation of de/centralization adopted by the legal, economic, and information systems literature with respect to the distinction between platforms and infrastructures. De/centralization in this case is linked to the concept of control.

The code-is-law interpretation recognizes that the blockchain network and its native coin can also be used as a legal enforcer of contracts and that the way in which these contracts are verified and enforced might impact their social value, validity and meanings. The concept of smart contract is mainly linked to this second techno-legal approach. A network can be more or less centralized based on the protocol used to verify and enforce claims on-chain. Whenever the value and significance of the claims held by any node depend on the specific way in which the contractual terms are understood and contingencies are verified (meaning that claims are incomplete and ambiguous), and on the specific identity of the nodes verifying, observing and interpreting these terms (meaning that the symbolic value and significance of claims can be subjective), the network is centralized. A decentralized network is a network where coins can be understood as complete, self-enforcing and unambiguous (context-independent) claims. This is the interpretation of de/centralization adopted by the cryptoinstitutionalist literature.

The principal-agent interpretation recognises that different nodes might treat their coins differently based on their own information, capabilities and roles. In this case, a network can be more or less centralized based on its degree of mutual dependence of the nodes and on the degree of the division of labour among those nodes. In this case, perfect decentralization emerges whenever nodes are completely symmetric so that they can do everything "in house" and do not need to rely or depend on the decisions of other nodes more specialised expertise or capabilities. Differently from the standard way in which markets and hierarchies are distinguished, decentralization in this case implies no division of labour at all.

The techno-legal interpretations of de/centralization reveal different aspects of the blockchain than the techno-economic approaches. However, it is interesting to notice that, even though each interpretation focusses on a different aspect of the blockchain, the three techno-legal 
interpretations of de/centralization can be understood as the legal equivalent of the three technoeconomic ones when the coin is treated as a generic right, rather than a valuable monetary tool.

The platform-logic interpretation is the equivalent of the protocol-logic interpretation: both define de/centralization by focussing on the fact that the value or significance of the coin reflects the value and significance of its underlying network and its technological characteristics. Different ways to control and affect the nature and value of the underling network have different implications on the value and significance of the native coin associated to it. The code-is-law interpretation is the equivalent of the permission-logic interpretation as both focus on the way in which the value and significance of the coin is assessed, verified, and validated. Different ways to maintain the integrity and validity of the coin have implications for its value and significance. The market-power interpretation is the equivalent of the principal-agent interpretation as they both focus on the concentration of activities and capabilities in few nodes and on the way in which this concentration of power (or distribution of labour) affects the value and significance of the coin.

This does not mean that the three techno-economic approaches are substantially a repetition of the three techno-legal approaches just in different sauce. Each interpretative approach of de/centralization focusses on its own peculiar mechanism and phenomena and on its specific blockchain aspect.

Table 6.5 and 6.6 recap the key characteristics of the techno-economic approach to de/centralization.

\begin{tabular}{|l|l|}
\hline $\begin{array}{l}\text { Techno-Legal second- } \\
\text { order interdependencies }\end{array}$ & $\begin{array}{l}\text { How the technological characteristics of the blockchain network shape and affect the significance of } \\
\text { the coin and the nature and origin of its legal claims and vice versa. }\end{array}$ \\
\hline \hline Approach & $\begin{array}{l}\text { Tries to understand how the claims embodied in the coin are endogenously shaped by the network } \\
\text { technology and vice versa: a change in the way in which the nodes can communicate and transact on- } \\
\text { chain and can verify, validate and append transactions directly affects the nature and legal significance } \\
\text { of the coin and vice versa. }\end{array}$ \\
\hline $\begin{array}{l}\text { Definition of } \\
\text { de/centralization }\end{array}$ & $\begin{array}{l}\text { How asymmetries in the social significance and meaning of coin (the symbolic dimension of a digital } \\
\text { network) depend on the asymmetries among the elements of the network (the technological } \\
\text { dimension) and vice versa. }\end{array}$ \\
\hline $\begin{array}{l}\text { Unit of analysis } \\
\text { (De/Centralization applies } \\
\text { to) }\end{array}$ & The blockchain network (\& protocol) \\
\hline \hline
\end{tabular}

Table 6.5: Overview of the techno-legal category of perspectives. 


\begin{tabular}{|c|c|c|c|}
\hline & Platform-logic & Code-is-law & Principal-agent \\
\hline & \multicolumn{3}{|c|}{ Decentralization } \\
\hline $\begin{array}{l}\text { Meaning of } \\
\text { decentralization }\end{array}$ & $\begin{array}{l}\text { Symmetric interdependence } \\
\text { between the symbolic dimension } \\
\text { and the technological dimension }\end{array}$ & $\begin{array}{l}\text { Symmetric interdependence between } \\
\text { the symbolic dimension and the } \\
\text { technological dimension }\end{array}$ & $\begin{array}{l}\text { Mainly independence of the } \\
\text { symbolic dimension from the } \\
\text { technological dimension }\end{array}$ \\
\hline $\begin{array}{l}\text { Condition for } \\
\text { decentralization }\end{array}$ & $\begin{array}{l}\text { Open and neutral network: } \\
\text { The value and significance of the } \\
\text { underlying network depends on the } \\
\text { technological characteristics of the } \\
\text { network (openness and neutrality to } \\
\text { other App-coins), and the value of } \\
\text { the coin depends on the value and } \\
\text { significance of the underlying } \\
\text { network. } \\
\text { However ,the reverse is also true: } \\
\text { the value and significance of the coin } \\
\text { affect the value underlying network } \\
\text { and this might determine the } \\
\text { technological nature of the network } \\
\end{array}$ & $\begin{array}{l}\text { Complete, unambiguous, and } \\
\text { objective claims: } \\
\text { The value and legal significance of the } \\
\text { coin are completely and } \\
\text { unambiguously defined ex-ante in the } \\
\text { network protocol and, for this reason, } \\
\text { claims can be enforced automatically } \\
\text { and autonomously } \\
\text { independently from the peculiar } \\
\text { contingencies and identity of any } \\
\text { specific ex-post enforcer or arbitrator }\end{array}$ & $\begin{array}{l}\text { No division of labour among the } \\
\text { nodes: } \\
\text { All nodes are endowed with the } \\
\text { same expertise, capabilities and } \\
\text { information so that each node is } \\
\text { identically able to carry out any } \\
\text { activity or decision } \\
\text { independently with no need to } \\
\text { outsource any activity to other } \\
\text { specialised nodes }\end{array}$ \\
\hline $\begin{array}{l}\text { Causality / } \\
\text { Directionality }\end{array}$ & Bidirectional / Symmetric & Bidirectional / Symmetric & Mainly absent (only indirect) \\
\hline Nature of claim & $\begin{array}{l}\text { Objective and technology- } \\
\text { dependent }\end{array}$ & $\begin{array}{l}\text { Complete, unambiguous, and } \\
\text { objective }\end{array}$ & $\begin{array}{l}\text { Objective and common to all } \\
\text { nodes }\end{array}$ \\
\hline \multirow[t]{2}{*}{$\begin{array}{l}\text { Implications for } \\
\text { the technological } \\
\text { sphere }\end{array}$} & Network as a general-purpose asset & $\begin{array}{l}\text { Network acting as a smart contract } \\
\text { enforcer }\end{array}$ & $\begin{array}{l}\text { Economic equality and self- } \\
\text { subsistence (liberty as } \\
\text { independence) of all nodes (no } \\
\text { economic dependence and no } \\
\text { specialization of roles) }\end{array}$ \\
\hline & \multicolumn{3}{|c|}{ Centralization } \\
\hline $\begin{array}{l}\text { Meaning of } \\
\text { centralization }\end{array}$ & $\begin{array}{l}\text { Asymmetric interdependence } \\
\text { between coins in the symbolic } \\
\text { dimension }\end{array}$ & $\begin{array}{l}\text { Asymmetric interdependence } \\
\text { between the symbolic dimension and } \\
\text { the technological dimension }\end{array}$ & $\begin{array}{l}\text { Asymmetric interdependence } \\
\text { between the symbolic dimension } \\
\text { and the technological dimension }\end{array}$ \\
\hline $\begin{array}{l}\text { Condition for } \\
\text { centralization }\end{array}$ & $\begin{array}{l}\text { Closed and coin-specific network: } \\
\text { The value of the native coin is } \\
\text { unilaterally pre-defined by the } \\
\text { network protocol and by the pre- } \\
\text { established range of DCoin and } \\
\text { DApps that are allowed on the } \\
\text { network. } \\
\text { The network discriminates among } \\
\text { different coins, thus generating } \\
\text { symbolic asymmetries among coins. }\end{array}$ & $\begin{array}{l}\text { Incomplete, ambiguous, and } \\
\text { subjective (context-dependent) } \\
\text { claims: } \\
\text { The value and legal significance of the } \\
\text { coin have to be established based on } \\
\text { the specific circumstances and, for } \\
\text { this reason, the nature of the claim } \\
\text { might vary based on the peculiar } \\
\text { contingencies and identity of the } \\
\text { specific ex-post enforcer or arbitrator }\end{array}$ & $\begin{array}{l}\text { Specialization and division of } \\
\text { labour among the nodes: } \\
\text { Nodes are endowed with } \\
\text { different expertise, capabilities } \\
\text { and information so each node } \\
\text { must outsource some activities } \\
\text { and decisions to other nodes so } \\
\text { that each node relies on others } \\
\text { in order to fulfil and carry out all } \\
\text { its required activities and } \\
\text { decision }\end{array}$ \\
\hline $\begin{array}{l}\text { Causality / } \\
\text { Directionality }\end{array}$ & $\begin{array}{l}\text { From the technological to the } \\
\text { symbolic dimension }\end{array}$ & $\begin{array}{l}\text { From the technological to the } \\
\text { symbolic dimension }\end{array}$ & Bidirectional / Asymmetric \\
\hline Nature of value & Objective and social & Subjective and contextual & Objective and social \\
\hline $\begin{array}{l}\text { Implications for } \\
\text { the technological } \\
\text { sphere }\end{array}$ & Network as a service-specific asset & $\begin{array}{l}\text { Presence of an enforcer and arbitrator } \\
\text { of last resort (i.e. a court or justice) }\end{array}$ & $\begin{array}{l}\text { The network as a system for the } \\
\text { efficient division of labour and } \\
\text { specialization of tasks }\end{array}$ \\
\hline
\end{tabular}

Table 6.6: Summary of the three techno-legal perspectives: the platform-logic, code-is-law, and principle-agent. 


\subsection{NT $\Leftrightarrow$ SC: Network Technology and Social Community}

The Ethereum project paved the way for the transformation of the original Bitcoin infrastructure into a proper platform for the operation of a variety of tokens supporting a variety of use cases. This opened up new perspectives on the blockchain that we called the techno-legal approach to decentralization. Differently from the Bitcoin infrastructure, the Ethereum platform enables the implementation of Touring-complete smart contracts for the automatic and autonomous enforcement of contracts between the various transacting parties.

On the $30^{\text {th }}$ of April 2016, nearly two years after the original conception of Ethereum, the DAO (decentralized Autonomous Organization) was launched in order to provide new decentralized business models to companies willing to offer services and products over the Ethereum platform. Few weeks after the project will be aborted and shut down due to an exploitation of a vulnerability of the DAO code that resulted in the theft of nearly $30 \%$ of the total number of ether coins raised by the crowdfunded token sale. A month later the majority of the Ethereum community decided to cancel the implicated transactions, to "bail out" the victims affected by the bug, and to create a fork in the Ethereum blockchain to restore a new status quo. This act substantially meant a redistribution of wealth among the community by fiat, in direct contrast with the protocol. As feared by the opponents to the decision, which will later create "Ethereum classic", this created a precedent: an ex-post purposeful intervention of a social community into the automated operations of the blockchain's code. Transactions that were "lawfully" approved and validated by the protocol were overruled on political ground, based on moral judgments entailing considerations of fairness, and rightfulness.

This event was not the first one of this kind. The Bitcoin community had already experienced something similar in 2013, when the bitcoin developers intervened to fix a hard fork that had developed in the bitcoin blockchain. But the Ethereum hard fork was certainly the first event to arise interest of the general public and that raised the attention of academics trying to understand the nature, the meaning and the implications of this act. Since the DAO collapse, the focus gradually started to shift from the rules according to which the coin is transacted among the nodes of the blockchain infrastructure (the techno-legal approach of "code is law"), to the rules and procedures followed by the members of the social community involved (more or less directly) in a certain blockchain ecosystem for the definition of the code, its update, amendment, abrogation, or change. Under this new approach, the focus of the analysis becomes the complex interaction between the social community of the members and the architectural design of the digital infrastructure.

In the literature this is commonly referred to as a shift from the inquiry on the on-chain governance of the blockchain to an inquiry on its off-chain governance. In reality, things are more complex than that as the two are strictly linked (Reijers et al., 2018). A proper focus on the nature of the infrastructure-social community relationship will better highlight the nature of this linkage. Other works distinguish between governance through or by the blockchain (on-chain) and governance of the blockchain (off-chain). The meaning of these two categorizations is substantially the same and denotes a shift from the network consensus protocol to the realm of the social community.

As noted by Reijers and colleagues (2018), while the first approach can be considered positive and procedural (it describes how the rule of code should work given a certain instrumental goal to attain), the second is normative and substantive in the sense that it involves normative value judgments by 
the part of political actors on what goal ought to be achieved and therefore, ultimately, on what ought to be done whenever a situation presents a choice that cannot be framed using the existing code of conduct and cannot be framed in objective and measurable (a-political) terms. For this reason, the works enquiring the infrastructure-social community relationship adopt a socio-technical or techno-political approach to decentralization. While the techno-legal approach inquires the procedures through which the positive law of code rules the transaction of the token on-chain, the techno-political approach is interested in the way in which the constitutional values of the social community are defined off-chain and embedded into the blockchain protocol. As De Fillippi and Hassan (2016) succinctly put it, "from code is law to law is code".

Pretty much all works belonging to this third interpretative stream recognise that, whenever unexpected crisis or events challenge the status quo and require the off-line intervention of the members of the social community, it is not sufficient to merely check the "on-chain" degree of centralization of centralization of a blockchain. Whenever the social community is involved off-chain to implement an institutional shift and so to establish a new constitutional order, the same dimensions characterising the degree of centralization of a blockchain on-chain can be used in order to understand the degree of centralization of the blockchain community off-chain. As a result, a new set of interpretations of centralization emerge: from a socio-technical or techno-political point of view, the degree of centralization is characterised by looking at the nature of the process adopted by the members of the social community in order to reach political (thus ethical) conclusions on the nature of the goal to be achieved, on the way in which the blockchain infrastructure ought to be normed and designed, and on the way in which this new constitutional order should be codified into a new legal framework. While the techno-legal approach inquiries decentralization through the lenses of a techno-legal interpretation of consensus, this third approach interprets decentralization by adopting a political and ethical interpretation of social consensus, while also trying to understand how the latter affects the former.

This third strand of the literature is the most recent one among the three and (maybe also for this reason) it is not possible to divide the various works into clear-cut groups. Many works dealing with the problems involved in the off-chain governance of the blockchain do not adopt a single point of view on the matter, but try to provide a broader overview of the problems generated. Yet, it is still possible to distinguish various ways in which centralization can be defined in function of the infrastructure-social community relationship.

\subsubsection{Social Community \& Information Sharing: Decentralization as Information Symmetries}

Information asymmetries denote imbalances in knowledge and therefore in power among the actors. Members who have more and earlier information have an advantage over those members knowing less and later. This advantage might create power imbalances that are often deemed to be unfair or illegal, as the practice of insider trading in corporate finance.

The problem of information imbalance represents a fundamental aspect in both previous dimensions: information asymmetries over the identity of the token holders might confer undue advantage (Coin $\Leftrightarrow$ Social Community), in the same way in which information asymmetries over the transaction patters of the various nodes do (Coin $\Leftrightarrow$ Network Technology). The same holds true with respect to the information over the state of a blockchain. Whenever a problem emerges on the 
blockchain, or some decisions have to be made, it is extremely likely that some members of the social community ("insiders") will know more than others and, potentially, exploit this information asymmetry at their personal advantage.

The literature presents various examples of this type (see Walch, 2019b). On the $17^{\text {th }}$ of September 2018, a bug in the bitcoin protocol was signalled by an anonymous reported to Bitcoin Core developers. These kept the vulnerability secret for nearly two days, before asking to all other members of the social community to upgrade their version of the protocol, first through public banners on bitcointalk and Reddit on the late evening of the $18^{\text {th }}$, then through a collective mailing list on the afternoon of the $19^{\text {th }} .^{40}$ The fact that some members of the social community knew the status of the network more than others might already be regarded as a source of power imbalance among the various members of the community.

A similar case occurred in October 2018 during an Ethereum conference held in Prague. On that occasion, ConsenSys organized a three-days meeting with around 20 Ethereum core developers in order to discuss future developments of Ethereum. Although written records of the meetings were produced, the event was not streamed online and all other members knew of the event only ex-post, thus manifesting their disappointment for how the situation was handled. ${ }^{41}$ Even in this case, some information on the state of the platform was circulated to some members before others could know, thus creating imbalances among the members of the community.

These examples highlight the fact that centralization can manifest itself as power relationship among the various members due to their different knowledge of the current and future states of the blockchain infrastructure and network. This aspect generates a parallelism with corporate law of publicly traded corporations, that prescribes that information about the state of the company should always be disclosed to the public and the hiding and manipulation of information by insiders is considered unlawful and illegal (see Hacker, 2017; Reyes, 2019).

This problem highlights two important facts. First, informational power depends greatly on the nature of the digital or physical space where meetings are held and information is shared. For the case of the Bitcoin bug, Bitcoin core developers used public platforms such as Reddit and private mailing lists, while in the case of the Ethereum-ConsenSys meeting, a participant admitted that one of the reasons why the meeting had to remain limited was that " $t$ ] here quite literally wasn't enough room for everyone. The spare breakout rooms at DevCon were designed for 10-15 people, and we had already brought in extra chairs to fit $20+.{ }^{\prime \prime 2}$ The relationship between the digital platforms where information can be exchanged (virtually unconstrained in space) and the members of the social community then becomes key in this respect. Second, the selection of certain members over others can reflect more or less formalised and institutionalised differences in the roles and status of the various members or developers of the social community. Both these points can play a role in defining the degree of centralization from a techno-political point of view.

\footnotetext{
${ }^{40}$ https://www.coindesk.com/the-latest-bitcoin-bug-was-so-bad-developers-kept-its-full-details-a-secret and https://bitcoincore.org/en/2018/09/20/notice/

${ }^{41}$ https://medium.com/@Irettig/how-open-is-too-open-bfc412cfod24

${ }^{42}$ https://medium.com/@Irettig/how-open-is-too-open-bfc412cf0d24
} 


\subsubsection{Social Community \& Information Sharing: Access and Control Symmetries}

For the various members of the community (stakeholders) to obtain the same information on the status of the blockchain infrastructure and network at nearly the same time, some place where information can be conveyed and shared becomes a necessary precondition. Differently from the blockchain infrastructure, where all information on the status of the network is instantaneously broadcasted to the whole network (and all its nodes), these off-chain information sharing places are platforms. These platforms can be more or less centralized based on their nature and characteristics. The degree to which any member of the community has the same rights of everyone else to access the platform, participate in the discussion, see and retrieve the same information that all others can access provides a further techno-political measure of centralization of a blockchain ecosystem. Decentralization in this case is defined by the degree of symmetry in the members' rights to access, share, and control information over these platforms. Ownership and control rights over these platforms play a significant role in ensuring perfect symmetry.

In the case of Bitcoin and Ethereum, multiple options are available. Not all of them are characterized by perfect symmetry in rights and control. ${ }^{43}$ Thus, GitHub and Reddit are both forums for opensource and user-generated content and they both have dedicated pages for both the Ethereum and the Bitcoin community. Both can be freely access (perfect symmetry in access rights), yet neither platform is technically open source: they are both owned and controlled by private corporations (Microsoft in the case of GitHub, Advance Publications Inc. in the case of Reddit). This means that, at least in theory, not only access can be potentially restrained, but information over both sites can be centrally controlled. So far, the freedom to access and circulate information on GitHub has proved problematic for countries willing to control access to the internet and implement censorship.

Other platforms can guarantee a higher degree of symmetry in rights. Bitcoin.org and Bitcoincore.org are two examples of open source projects where information about the status of the Bitcoin network is shared. The mailing list bitcoin-dev requires a subscription and registration, and is moderated by third parties, while a list of all subscribed members and their activities is available to the mailing list administrator. Other open source projects providing discussion forums (such as freenode.net) require a preliminary registration of the users. Some platforms and discussion forums are proprietary others are owned by third parties: some forums are available over private platforms, such as forums on cryptocompare.com.

In sum, even though the information on-chain can be equally accessible and transferred by all members, this might not be the case for the off-chain access and transmission of information: ecosystems relying on platforms that present an asymmetric distribution of rights for the access and transmission of information among the various members of the social community will be characterised by a higher degree of centralization. Even though the blockchain infrastructure is censorship-resistant and tamper resistant, information off-chain can be censored and tampered with, depending on the nature of the platforms over which information is shared and transmitted. Information decentralization on-chain can very well be matched with high information centralization off-chain. Differently from the previous case, in this case information asymmetry does not derive

\footnotetext{
${ }^{43}$ A partial list can be found at https://bitcoin.org/en/development\#code-review and https://cointelegraph.com/bitcoin-for-beginners/where-to-discuss-bitcoin\#reddit.
} 
from an informal imbalance of powers, but from a clear imbalance over the rights of the members over the off-chain information platforms.

\subsubsection{Social Community \& Participation and Decision Rights: On-Chain Modification Symmetries}

No code is for ever, and neither is a blockchain protocol. Code has to be upgraded, changed, or amended. The distribution of the rights defining who is allowed to propose, accept or implement these changes can provide a further insight on the degree of centralization of a blockchain ecosystem. These distinctions in the formal roles of the various members of the community are often formalised and institutionalised. For these reasons, these types of asymmetries can be treated as decision rights held by the various members of the community. Accountability often comes with these rights.

Both bitcoin and Ethereum present a two-layer hierarchical structure when it comes to their community of developers. On the one hand, proposals follow an open source logic: anyone can propose modifications and upgrades to the codes. These suggestions and proposals can be made on any of the dedicated forum or discussion platforms, but they are mainly made on GitHub, which is an open source, user-generated platform (yet privately owned). As discussed earlier, suggestions can also be made internally, using dedicated channels (such as Bitcoincore.org). However, only a restricted set of core developers are in charge for the verification, validation, acceptance and implementation of the changes to the blockchain protocol. This narrow set of actors usually corresponds to the moderators or administrators of platforms, such as Bitcoincore.org.

The overwhelming majority of public blockchain presents this type of hierarchical structure of their social members and programmers, formalising and institutionalising the distinction between core coders and all other open source contributors. Only the former is responsible and formally accountable for taking the last decisions. As only core developers are entrusted with decision rights, or residual rights, they substantially act as de facto managers, or owners (depending on the perspective) of the blockchain. Other authors suggest they should be treated as trusted fiduciaries (Hacker, 2017; Reyes, 2019; Rodrigues, 2018; Walch, 2018).

The distinction between the different types of coders represents one of the most inquired aspects of the literature on off-chain governance and for this reason it does not require much discussion (Bodo and Giannopoulos 2019; De Filippi and Loveluck, 2016; Reyes, 2019; Rodrigues, 2018; Walch, 2018, 2019; Ziolkowski et al 2018a). Core developers act as the ultimate gatekeepers and guardians of the blockchain code as they are the ones endowed with the exclusive rights to take the last decisions on what versions of the protocol should be executed, and which one should be rejected. The more restricted and narrower the circle of core developers vis-à-vis the totality of the members of the social community, the more centralized a blockchain ecosystem is. De Filippi and Loveluck (2016) distinguish between two main types or configurations: "democratic-organic" versus "autocraticmechanistic". A fully democratic (thus decentralized) arrangement would be one "fully open", where anyone willing to participate in the proposal and choice of the new protocol would be allowed to. A more centralized situation would be one where even proposals should come from a restricted set of pre-authorised members. Blockchain communities are more or less centralized based on their degree of openness and participation, in the same way in which (on-chain) blockchain infrastructures are. 
More generally, the greater number of members of the social community who decide to contribute, the greater the degree of decentralization. Under this paradigm, maximum centralization would ensue in the case of a single benevolent dictator deciding for everyone (Azouvi et al., 2018; Beck et al., 2018).

The dichotomy between proposals and acceptance of the protocol (off-chain) play a similar role of the dichotomy between execution and verification of a transaction (on-chain). From a techno-legal point of view (Coin $\Leftrightarrow$ Network Technology), some blockchain networks might only grant access to the infrastructures and its transactions to verified and authorised members, in the same way in which a blockchain community might only authorise some members to join the discussion and submit proposals from a techno-political (or socio-technical) point of view. Similarly, in the same way in which a blockchain can operate through trusted gateways on-chain for the verification and validation of transactions modifying the on-chain status of the blockchain infrastructure (technolegal point of view), there might be trusted gateways off-chain entrusted with the rights to certify and validate the modification of the "constitutional" status quo off-chain (from a techno-political point of view). The two-stage framework characterising the techno-legal dimension of on-chain governance is similar to the two-layered architecture characterising the socio-technical dimension of off-chain governance.

In any case, complete decentralization on-chain (form a techno-legal perspective) is completely independent from, and does not necessarily imply decentralization off-chain (from a techno-political point of view).

\subsubsection{Social Community \& Political Power: Decentralization as Capacity to Influence}

The formal or institutionalised distinctions among the various members of the community do not explain why or how core developers are selected in the first place, neither does it rule out informal powers held by the different actors (regardless of their formal prerogatives). We can treat both aspects at the same time, and group them under the same classification: sources of informal power of the various members of the community deriving from their capacity to influence the decisions of other actors of the community. There might be different reasons for this source of power.

First, power might derive from asymmetries in capabilities recognised and appreciated by the community. One developer might be regarded as particularly more capable than the others. This might generate some reputational mechanism, so that the opinions of a reputable developer are regarded more highly than the opinion of others. Highly regarded developers might have much more informal power than others when it comes to influencing the overall community. This reputation might be formalised or not. Communities with fewer influential and reputable voices will tend to be more centralized as they will depend on fewer individuals, as compared to communities where reputation is evenly spread among a greater number of members. Asymmetry in perceived (or real) capabilities and reputation confers power: the higher this asymmetry, the higher the power of individual members, the higher the overall degree of centralization.

Second, some members might have more social legitimacy than others. Legitimacy might be independent from reputation or capabilities. Founders or early developers might have more legitimacy in the eyes of the members of the social community as they have shown greater and longer commitment to the project, regardless of their actual level of capabilities. Blockchains where there is one single well-identifiable funder or group of founders are more centralized than others 
where a leading figure is missing. From this point of view, nearly all blockchain projects are more centralized than the bitcoin one, and this is especially true for Ethereum. As a result, asymmetries in perceived legitimacy among the different members confer power: the higher this asymmetry, the higher the power of single individual members, the higher the overall degree of centralization of the social ecosystem.

Third, some members might have more exposure or notoriety than others. Vocal members might just be more influential than others, everything else equal. Notoriety might derive from involvement in other institutions (academic, industry or professional bodies, political), news outlets or community forums. High exposure might generate the same type of informal power than the other two aspects.

In sum, the degree of centralization of a certain decision-making process might also depend on the differential political power of some members to influence others.

It should be noted that these sources of power depend on the possibility to identify a particular contributor, to recognise him or her, and to distinguish their advice or opinion from the ones of the others. Asymmetries in legitimacy, reputation, notoriety and exposure therefore depend on the lack of anonymity of the various members of the community. Pseudonymity might still work, as long as pseudonyms remain unique and univocally identify a single member (but they might not work for some other sources of power, such as the obtainment of predefined community roles that presuppose full disclosure of the identity of the member). As a result, even though on-chain anonymity and pseudonymity are the necessary precondition for on-chain decentralization from a socio-economic and techno-legal point of view, the revelation of the members' identity off-chain is the condition that addresses some governance problems off-chain. Yet, this also increases the level of off-chain centralization as it generates asymmetries in power, as Satoshi Nakamoto correctly understood in 2008.

While governance from a techno-legal and socio-economic point of view might still support privacy, governance from a techno-political or socio-technical point of view heavily depends on the social identity of the actors, and for this reason it might break-down in a regime of privacy protection and anonymous members. In any case, what is important to underline here is that full anonymity from a techno-legal point of view (on-chain) does not imply anonymity and therefore decentralization from a techno-political point of view (off-chain).

\subsubsection{Summary of Socio-Technical / Techno-Political Approach}

As recognised by many authors, whenever circumstances change and a crisis perturbs the status quo, the positivist approach of the "code is law" philosophy cannot provide any guidance on how a system should operate a transition from one institutional arrangement to another one. Someone has to act as a decision maker. This decision can be reached in various ways and according to different procedures. These procedures can be characterised by different degrees of centralization depending on the different degrees of asymmetries characterising the relationship between the political and ethical dimension of the blockchain social community and the technological dimension of the blockchain infrastructure.

These techno-political or techno-ethical sources of asymmetries simply retrace the sources of asymmetries characterising the techno-legal dimension of decentralization, with the difference that 
now asymmetries do not arise from the interaction between the blockchain infrastructure and the digital world, but from the blockchain infrastructure and the real world: information asymmetries among members of the social community over the current state of the infrastructure protocol, asymmetries in access and decision rights among members of the social community over the future nature of the infrastructure protocol, asymmetries in the identities of the members of the social community with respect to their political influence, perceived capabilities, legitimacy, reputation and notoriety.

\section{Conclusion}

The aim of this working paper has been to initiate a debate on de/centralization in digital networks by drawing on an in-depth study of blockchain networks. The paper first set out the scene for the study by highlighting the dual nature of digital platforms and -infrastructures and the diversity in the academic debate on the understanding of the two phenomena. The paper then proposed a conceptual framework outlining the de/centralization in digital networks across the dimensions of use and access, and promoted the notion of first-order and second-order interdependencies. This framework was projected onto the context of blockchain networks and formulated as a framework for the analysis of de/centralization in blockchain networks. This blockchain de/centralization framework was applied in an in-depth analysis of second-order blockchain interdependencies. The paper contributes to a significant refinement of the often coarse discussion of decentralization in blockchain networks, and it is our hope that the findings in the future will help contribute to a more comprehensive understanding of digital platforms and -infrastructures in general. 


\section{Appendix A: Approach}

As part of an eight-month project initiated in February 2018 to write an online cryptocurrency innovation course, we undertook a comprehensive effort to seek out the blockchain literature from across a number of academic communities. This report has undertaken an explorative analysis of the core blockchain conceptualisations applied in the reviewed papers, and through this seeks to understand the theoretical foundation explored in these academic works. This report is, therefore, not strictly a bibliometric study of blockchain research, but a theoretical paper, which relates and contextualises the findings to extant blockchain research. The following seeks to contextualise the corpus from which our core ideas have emerged.

We have collected 425 academic articles directly related to blockchain and cryptoassets. Table A.1 lists the main journals and conferences represented in the corpus. We have, in addition, sourced selected unpublished working papers and central bank whitepapers from prolific blockchain observers, such as from the Bank of England and the US Federal Reserve. As the conceptual grounding of blockchain within Information Systems is still in its infancy, we found it important to take a broad rather than narrow view when searching for literature discussing the phenomenon. The text mining software package Leximancer (Smith and Humphreys, 2006; Thomas, 2014) offers a means of exploring and visualising large texts in order to elicit concepts and categories. This has been used to the study of a variety of subjects, for example, academic publications (Cretchley et al., 2010; Wuehrer and Smejkal, 2013; Anagnostopoulos and Bason, 2015; Randhawa et al., 2016). A textual analysis using Leximancer of the contents of the 425 journal articles forming the basis for our work in this report shows the variety of core concepts covered in the corpus. In particular, the analysis demonstrates the variety of core issues discussed in the literature, spanning technical discussions on what constitute a blockchain, the legal aspects, the critical issue of currency and value, the relationship to financial systems and regulation, and the relationship to smart contracts (See Figure A.1).

\begin{tabular}{|c|c|c|}
\hline $\begin{array}{l}\text { American Behavioural Scientist } \\
\text { Applied Economics } \\
\text { Cambridge Journal of Economics } \\
\text { Cato Journal } \\
\text { Columbia Science \& Technology Law } \\
\quad \text { Review } \\
\text { Computer Law \& Security Review } \\
\text { Conference on Privacy \& Trust } \\
\text { Decision Support Systems } \\
\text { Designs, Codes and Cryptography } \\
\text { Economic Letters } \\
\text { Economic Modelling } \\
\text { Electronic Commerce Research \& } \\
\quad \text { Applications } \\
\text { Emerging Markets Finance \& Trade } \\
\text { Engineering Science, Technology, and } \\
\quad \text { Society } \\
\text { Environment and Planning D } \\
\text { European Conference of Information } \\
\quad \text { Systems } \\
\text { Expert Systems with Applications } \\
\text { Finance Research Letters } \\
\text { First Monday } \\
\text { Future Generation Computer Systems } \\
\text { Games } \\
\text { Hawaiian International Conference on } \\
\quad \text { Information Systems } \\
\text { IEEE Access } \\
\text { IEEE Computer Society } \\
\text { Information \& Management } \\
\text { Information \& Organization } \\
\text { Information Economics and Policy }\end{array}$ & $\begin{array}{l}\text { International Advances in Economic } \\
\text { Research International Journal } \\
\text { International Journal of Computer } \\
\text { Engineering \& Technology } \\
\text { International Journal of Law and } \\
\text { Information Technology } \\
\text { International Review of Intellectual } \\
\text { Property and Competition Law } \\
\text { International Review of Sociology } \\
\text { Internet Policy Review } \\
\text { Journal of Economic Behaviour \& } \\
\text { Organization } \\
\text { Journal of Economic Interaction and } \\
\text { Coordination } \\
\text { Journal of Financial Crime } \\
\text { Journal of Financial Stability } \\
\text { Journal of Information Technology } \\
\text { Journal of International Financial } \\
\text { Markets, Institutions \& Money } \\
\text { Journal of Legislation and Public Policy } \\
\text { Journal of Management Information } \\
\text { Systems } \\
\text { Journal of Money Laundering Control } \\
\text { Journal of the Association of } \\
\text { Information Systems } \\
\text { Journal of Theoretical and Applied } \\
\text { Electronic Commerce Research } \\
\text { Journal of Information Security and } \\
\text { Applications } \\
\text { Ledger Journal } \\
\text { MIS Quarterly Executive } \\
\text { Nebraska Law Review }\end{array}$ & $\begin{array}{l}\text { New Media \& Society } \\
\text { Organization } \\
\text { OzChi Physica A } \\
\text { Pittsburgh Tax Review } \\
\text { Police Journal } \\
\text { Procedia Economics and Finance } \\
\text { Proceedings on Privacy Enhancing } \\
\quad \text { Technologies } \\
\text { Research in International Business and } \\
\quad \text { Finance } \\
\text { SMU Science and Technology Law } \\
\quad \text { Review } \\
\text { Social Semiotics } \\
\text { Technology Forecasting \& Social Change } \\
\text { Telecommunications Policy } \\
\text { Telematics and Informatics } \\
\text { The George Washington Law Review } \\
\text { The Independent Review } \\
\text { The Journal of Private Enterprise } \\
\text { The Policy Journal } \\
\text { The Quarterly Review of Economics and } \\
\text { Finance } \\
\text { The Review of Austrian Economics } \\
\text { Theoretical and Applied Economics } \\
\text { Theoretical Inquiries in Law } \\
\text { Theory, Culture \& Society } \\
\text { University of Illinois Law Review } \\
\text { Vanderbilt Journal of Entertainment \& } \\
\text { Technology Law } \\
\text { Virginia Tax Review } \\
\text { William \& Mary Law Review } \\
\text { Yale Journal on Regulation }\end{array}$ \\
\hline
\end{tabular}

Table A.1: Overview of the academic outlets represented in the corpus of papers 

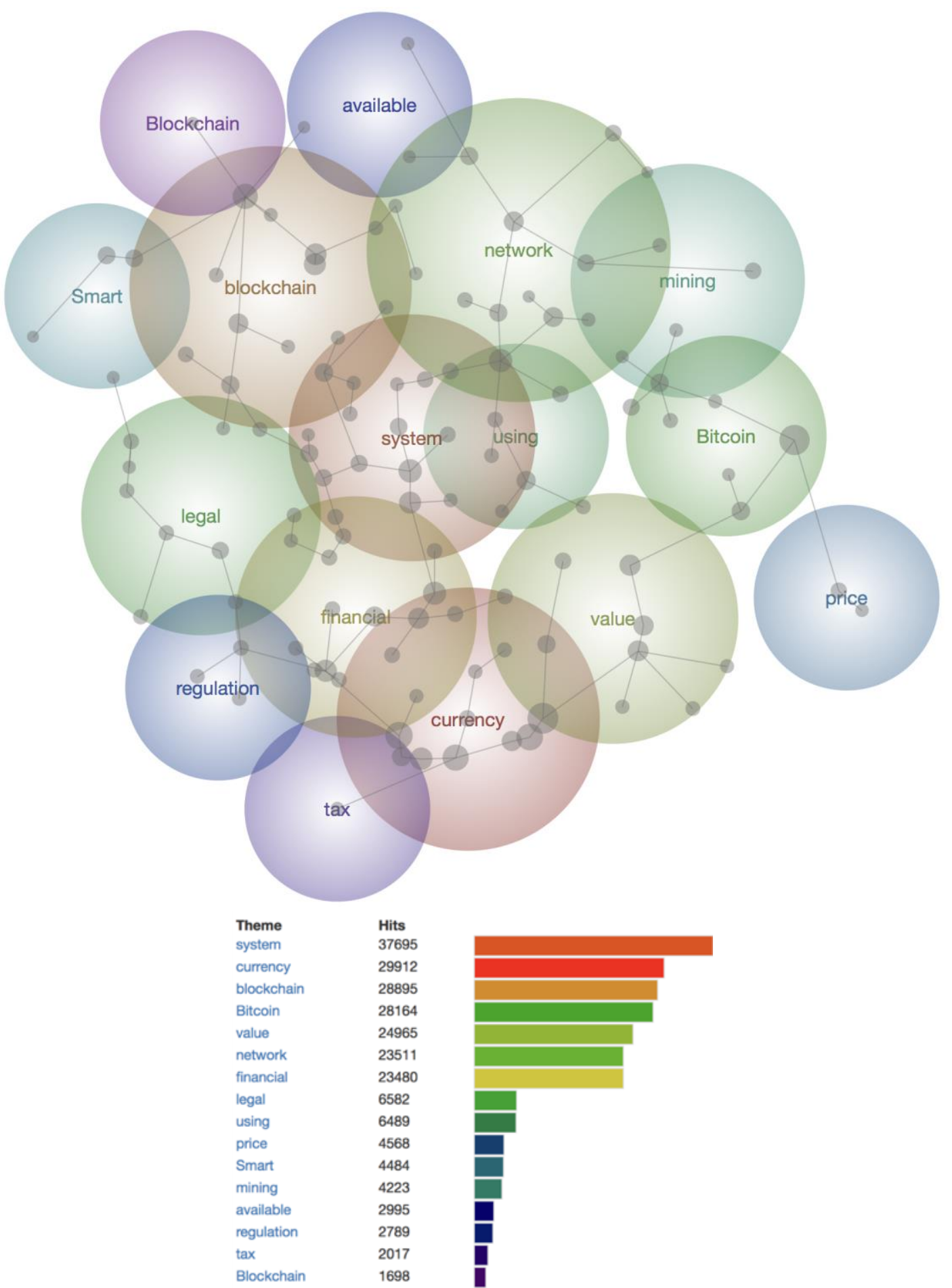

Figure 1: Leximancer concept map illustrating the core concepts as spheres reflecting connected concepts (top), and frequency of concepts (bottom). 


\section{References}

Abadi, J. \& M. Brunnermeier (2018): Blockchain economics. National Bureau of Economic Research. 0898-2937.

Abdelnour, S., H. Hasselbladh, \& J. Kallinikos (2017): Agency and institutions in organization studies. Organization studies, vol. 38, no. 12, pp. 1775-1792.

Abramaowicz, M. (2016): Cryptocurrency-based law. Ariz. L. Rev., vol. 58, pp. 359.

Adhami, S., G. Giudici, \& S. Martinazzi (2018): Why do businesses go crypto? An empirical analysis of initial coin offerings. Journal of Economics and Business, vol. 100, pp. 64-75.

Adner, R. (2017): Ecosystem as structure: an actionable construct for strategy. Journal of management, vol. 43 , no. 1 , pp. 39-58.

Agre, P. E. (2003): P2p and the promise of internet equality. Communications of the ACM, vol. 46, no. 2, pp. 39-42.

Akhshabi, S. \& C. Dovrolis (2013): The evolution of layered protocol stacks leads to an hourglassshaped architecture. In Dynamics On and Of Complex Networks, Volume 2, ed. A. Mukherjee, M. Choudhry, F. Peruani, N. Ganguly, and B. Mitra. Heidelberg: Birkhäuser, pp. 55-88.

Alaimo, C. \& J. Kallinikos (2017): Computing the everyday: Social media as data platforms. The Information Society, vol. 33, no. 4, pp. 175-191.

Ali, R., J. Barrdear, R. Clews, \& J. Southgate (2014): Innovations in payment technologies and the emergence of digital currencies. Bank of England Quarterly Bulletin, pp. Q3.

Ali, R., J. Barrdear, R. Clews, \& J. Southgate (2014): The economics of digital currencies. Bank of England Quarterly Bulletin, pp. Q3.

Ametrano, F. M. (2016): Hayek money: The cryptocurrency price stability solution. Available at SSRN 2425270.

Ammous, S. (2018): Can cryptocurrencies fulfil the functions of money? The Quarterly Review of Economics and Finance, vol. 70, pp. 38-51.

Anagnostopoulos, C. \& T. Bason (2015): Mapping the first 10 years with Leximancer: themes and concepts in the sports management international journal Choregia. SMIJ, vol. 11, no. 1.

Andersson Schwarz, J. (2017): Platform Logic: An Interdisciplinary Approach to the Platform-Based Economy. Policy \& Internet, vol. 9, no. 4, pp. 374-394.

Arjaliès, D.-L. (2019): 'At the Very Beginning, There's This Dream.'The Role of Utopia in the Workings of Local and Cryptocurrencies. Forthcoming in the Palgrave "Handbook of Alternative Finance," edited by Raghavendra (Raghu) Rau and Robert Wardrop.

Arruñada, B. (2012): Institutional foundations of impersonal exchange: Theory and policy of contractual registries. University of Chicago Press. 0226028321.

Arruñada, B. (2018): Blockchain's struggle to deliver impersonal exchange. Minn. JL Sci. \& Tech., vol. 19 , pp. 55.

Arruñada, B. \& L. Garicano (2018): Blockchain: The birth of decentralized governance. Pompeu Fabra University, Economics and Business Working Paper Series, vol. 1608.

Arthur, W. B. (1989): Competing technologies, increasing returns, and lock-in by historical events. The Economic Journal, vol. 99, no. 394, pp. 116-131.

Atzori, M. (2015): Blockchain technology and decentralized governance: Is the state still necessary? Available at SSRN 2709713.

Azouvi, S., M. Maller, \& S. Meiklejohn Egalitarian society or benevolent dictatorship: The state of cryptocurrency governance. In. Springer, pp. 127-143.

Baldwin, C. Y. (2012): Organization design for business ecosystems. Journal of Organization Design, vol. 1 , no. 1.

Ballandies, M. C., M. M. Dapp, \& E. Pournaras (2018): Decrypting Distributed Ledger DesignTaxonomy, Classification and Blockchain Community Evaluation. arXiv preprint arXiv:1811.03419. 
Ballandies, M. C., M. M. Dapp, \& E. Pournaras (2018): Decrypting Distributed Ledger DesignTaxonomy, Classification and Blockchain Community Evaluation. arXiv preprint arXiv:1811.03419.

Baran, P. (1964): On distributed communications networks. IEEE transactions on Communications Systems, vol. 12, no. 1, pp. 1-9.

Barrdear, J. \& M. Kumhof (2016): The macroeconomics of central bank issued digital currencies.

Baur, D. G., K. Hong, \& A. D. Lee (2018): Bitcoin: Medium of exchange or speculative assets? Journal of International Financial Markets, Institutions and Money, vol. 54, pp. 177-189.

Beck, R., C. Müller-Bloch, \& J. L. King (2018): Governance in the Blockchain Economy: A Framework and Research Agenda. Journal of the Association for Information Systems, vol. 19, no. 10, pp. 1020-1034.

Beck, R., C. Müller-Bloch, \& J. L. King (2018): Governance in the blockchain economy: A framework and research agenda. Journal of the Association for Information Systems, vol. 19, no. 10, pp. 1020-1034.

Benkler, Y. (2006): The wealth of networks: How social production transforms markets and freedom. Yale University Press. 0300127235.

Benlian, A., D. Hilkert, \& T. Hess (2015): How open is this Platform? The meaning and measurement of platform openness from the complementers' perspective. Journal of Information Technology, vol. 30, no. 3, pp. 209-228.

Biais, B., C. Bisiere, M. Bouvard, \& C. Casamatta (2019): The blockchain folk theorem. The Review of Financial Studies, vol. 32, no. 5, pp. 1662-1715.

Birch, D. (2017): Before Babylon, Beyond Bitcoin: From Money that We Understand to Money that Understands Us London Publishing Partnership, UK.

Bjerg, O. (2016): How is bitcoin money? Theory, Culture \& Society, vol. 33, no. 1, pp. 53-72.

Blandin, A., A. S. Cloots, H. Hussain, M. Rauchs, R. Saleuddin, J. G. Allen, K. Cloud, \& B. Z. Zhang (2019): Global Cryptoasset Regulatory Landscape Study. Available at SSRN.

Böhme, R., N. Christin, B. Edelman, \& T. Moore (2015): Bitcoin: Economics, technology, and governance. Journal of Economic Perspectives, vol. 29, no. 2, pp. 213-38.

Budish, E. (2018): The economic limits of bitcoin and the blockchain. National Bureau of Economic Research.

Buterin, V. (2014): A next-generation smart contract and decentralized application platform. white paper, vol. 3, pp. 37.

Buterin, V. (2014b): Ethereum Scalability and Decentralization Updates. BlogEthereum, Available at: https://blog.ethereum.org/2014/02/18/ethereum-scalability-and-decentralization-updates/

Buterin, V. (2017): The Meaning of Decentralization. Medium. https://medium.com/@VitalikButerin/the-meaning-of-decentralization-a0c92b76a274

Buterin, V. (2018): Governance, part 2: Plutocracy is still bad. Vitalik Buterin's Website, vol. 28.

Campbell-Verduyn, M. (2017): Bitcoin and beyond: Cryptocurrencies, blockchains, and global governance. Routledge. 1351814079.

Carney, M. The future of money. In.

Catalini, C. \& J. S. Gans (2016): Some simple economics of the blockchain. National Bureau of Economic Research.

Chaum, D. (2003): Untraceable electronic mail, return addresses and digital pseudonyms. In Secure electronic votingSpringer, pp. 211-219.

Chaum, D. Blind signatures for untraceable payments. In. Springer, pp. 199-203.

Chaum, D., A. Fiat, \& M. Naor Untraceable electronic cash. In. Springer, pp. 319-327.

Cheah, E.-T. \& J. Fry (2015): Speculative bubbles in Bitcoin markets? An empirical investigation into the fundamental value of Bitcoin. Economics Letters, vol. 130, pp. 32-36.

Chiu, J. \& T. V. Koeppl (2017): The economics of cryptocurrencies-bitcoin and beyond. Available at SSRN 3048124.

Chu, S. \& S. Wang (2018): The Curses of Blockchain Decentralization. arXiv preprint arXiv:1810.02937. 
Ciborra, C., K. Braa, A. Cordella, V. Hepsø, B. Dahlbom, A. Failla, \& O. Hanseth (2000): From control to drift: the dynamics of corporate information infrastructures. Oxford University Press on Demand. 0198297343.

Close, F. (2001): Lucifer's Legacy: The Meaning of Asymmetry. Oxford Paperbacks.

Coase, R. H. (1937): The nature of the firm. economica, vol. 4, no. 16, pp. 386-405.

Coase, R. H. (1960): The problem of social cost. In Classic papers in natural resource economicsSpringer, pp. 87-137.

Cong, L. W. \& Z. He (2019): Blockchain disruption and smart contracts. The Review of Financial Studies, vol. 32, no. 5, pp. 1754-1797.

Constantinides, P., O. Henfridsson, \& G. G. Parker (2018): Special Issue Introduction: Platforms and Infrastructures in the Digital Age. Information Systems Research, vol. 29, no. 2, pp. 381-400. https://pubsonline.informs.org/doi/pdf/10.1287/isre.2018.0794

Corbet, S., A. Meegan, C. Larkin, B. Lucey, \& L. Yarovaya (2018): Exploring the dynamic relationships between cryptocurrencies and other financial assets. Economics Letters, vol. 165, pp. 28-34.

Corbet, S., B. Lucey, A. Urquhart, \& L. Yarovaya (2019): Cryptocurrencies as a financial asset: A systematic analysis. International Review of Financial Analysis, vol. 62, pp. 182-199.

Corradi, F. \& P. Höfner (2018): The disenchantment of Bitcoin: unveiling the myth of a digital currency. International Review of Sociology, vol. 28, no. 1, pp. 193-207.

Cretchley, J., D. Rooney, \& C. Gallois (2010): Mapping a 40-year history with Leximancer: Themes and concepts in the Journal of Cross-Cultural Psychology. Journal of Cross-Cultural Psychology, vol. 41, no. 3, pp. 318-328.

Croman, K., C. Decker, I. Eyal, A. E. Gencer, A. Juels, A. Kosba, A. Miller, P. Saxena, E. Shi, \& E. G. Sirer On scaling decentralized blockchains. In. Springer, pp. 106-125.

Cusumano, M. A., D. B. Yoffie, \& A. Gawer (2019): The Business of Platforms: Strategy in the Age of Digital Competition, Innovation, and Power. HarperCollins Publishers. 0062896326.

Dai, W. (1998): B-money. Consulted, vol. 1, pp. 2012.

Davidson, S., P. De Filippi, \& J. Potts (2016): Disrupting governance: The new institutional economics of distributed ledger technology. Available at SSRN 2811995.

Davidson, S., P. De Filippi, \& J. Potts (2016): Economics of blockchain. Available at SSRN 2744751.

Davidson, S., P. De Filippi, \& J. Potts (2018): Blockchains and the economic institutions of capitalism. Journal of Institutional Economics, vol. 14, no. 4, pp. 639-658.

De Filippi, P. (2016): The interplay between decentralization and privacy: the case of blockchain technologies. Journal of Peer Production, Issue, no. 7.

De Filippi, P. \& B. Loveluck (2016): The invisible politics of bitcoin: governance crisis of a decentralized infrastructure. Internet Policy Review, vol. 5, no. 4.

De Filippi, P. \& G. McMullen (2018): Governance of blockchain systems: Governance of and by Distributed Infrastructure.

De Filippi, P. \& S. Hassan (2018): Blockchain technology as a regulatory technology: From code is law to law is code. arXiv preprint arXiv:1801.02507.

De Filippi, P. \& A. Wright (2018): Blockchain and the Law: The Rule of Code. Harvard University Press. 0674985915.

de Reuver, M., C. Sørensen, \& R. Basole (2018): The Digital Platform: A Research Agenda. Journal of Information Technology, vol. 33, no. 2, pp. 124-135. http://link.springer.com/article/10.1057/s41265-016-0033-3

De Rossi, L. M., N. Abbatemarco, \& G. Salviotti Towards a Comprehensive Blockchain Architecture Continuum.

Deibert, R., J. Palfrey, R. Rohozinski, \& J. Zittrain (2010): Access controlled. The Shaping of Power, Rights, and Rule in Cyberspace, Cambridge, Massachusetts, London, England.

Dodd, N. (2005): Laundering "money": on the need for conceptual clarity within the sociology of money. European Journal of Sociology/Archives Européennes de Sociologie, vol. 46, no. 3, pp. 387-411. 
Dodd, N. (2016): The social life of money. Princeton University Press. 1400880866.

Dodd, N. (2018): The social life of Bitcoin. Theory, culture \& society, vol. 35, no. 3, pp. 35-56.

DuPont, Q. (2019): Cryptocurrencies and Blockchains. John Wiley \& Sons.

DuPont, Q. 8 Experiments in algorithmic governance. Bitcoin and Beyond, pp. 157.

Dwyer, G. P. (2015): The economics of Bitcoin and similar private digital currencies. Journal of Financial Stability, vol. 17, pp. 81-91.

Easley, D., M. O'Hara, \& S. Basu (2019): From mining to markets: The evolution of bitcoin transaction fees. Journal of Financial Economics.

Eaton, B. D., S. Elaluf-Calderwood, C. Sørensen, \& Y. Yoo (2015): Distributed Tuning of Boundary Resources: The Case of Apple's iOS Service System. MIS Quarterly: Special Issue on Service Innovation in a Digital Age, vol. 39, no. 1, pp. 217-243.

Eisenmann, T. R., G. Parker, \& M. Van Alstyne (2009): Opening platforms: how, when and why? Platforms, markets and innovation, vol. 6, pp. 131-162.

Epstein, D., C. Katzenbach, \& F. Musiani (2016): Doing internet governance: practices, controversies, infrastructures, and institutions. Internet Policy Review, vol. 5, no. 3.

Evans, D. S. \& R. Schmalensee (2005): The industrial organization of markets with two-sided platforms. National Bureau of Economic Research. NBER Working Paper No. 11603. September.

Evans, D. S. \& R. Schmalensee (2016): The Matchmakers: The New Economics of Multisided Platforms Boston: Harvard Business Review Press.

Eyal, I. \& E. G. Sirer (2018): Majority is not enough: Bitcoin mining is vulnerable. Communications of the ACM, vol. 61, no. 7, pp. 95-102.

Faulkner, P. \& J. Runde (2019): Theorizing the Digital Object. MIS Quarterly, vol. 43, no. 4.

Finck, M. (2018): Blockchain regulation and governance in Europe. Cambridge University Press. 1108616569.

Finney, H. (1993), Digital Cash \& Privacy, Satoshi Nakamoto institute, retrieved from https://nakamotoinstitute.org/digital-cash-and-privacy/

Flew, T. (2019): The platformized Internet: Issues for Internet law and policy. Journal of Internet Law, vol. 22 , no. 11 , pp. 3-16.

Fransman, M. (2010): The new ICT ecosystem: Implications for policy and regulation. Cambridge University Press. 1139484141.

Fuchs, C. (2011): Web 2.0, prosumption, and surveillance. Surveillance \& Society, vol. 8, no. 3, pp. 288-309.

Fuchs, C. (2013): Internet and surveillance: The challenges of Web 2.0 and social media. vol. 16Routledge. 1136655271.

Gandal, N. \& H. Halaburda (2014): Competition in the cryptocurrency market.

Gandal, N. \& H. Halaburda (2016): Can we predict the winner in a market with network effects? Competition in cryptocurrency market. Games, vol. 7, no. 3, pp. 16.

Gans, J. S. \& H. Halaburda (2015): Some economics of private digital currency. In Economic Analysis of the Digital EconomyUniversity of Chicago Press, pp. 257-276.

Gawer, A. (2009): Platform dynamics and strategies: from products to services. Platforms, markets and innovation, vol. 45, pp. 57.

Gawer, A. (2011): Platforms, markets and innovation. Edward Elgar Publishing.

Gawer, A. (2014): Bridging differing perspectives on technological platforms: Toward an integrative framework. Research Policy, vol. 43, no. 7, pp. 1239-1249.

Gawer, A. \& R. Henderson (2007): Platform owner entry and innovation in complementary markets: Evidence from Intel. Journal of Economics \& Management Strategy, vol. 16, no. 1, pp. 1-34.

Gencer, A. E., S. Basu, I. Eyal, R. Van Renesse, \& E. G. n. Sirer (2018): Decentralization in bitcoin and ethereum networks. arXiv preprint arXiv:1801.03998.

Gencer, A. E., S. Basu, I. Eyal, R. Van Renesse, \& E. G. Sirer (2018): Decentralization in bitcoin and ethereum networks. arXiv preprint arXiv:1801.03998. 
Gervais, A., G. O. Karame, K. Wüst, V. Glykantzis, H. Ritzdorf, \& S. Capkun On the security and performance of proof of work blockchains. In. ACM, pp. 3-16.

Gervais, A., G. O. Karame, V. Capkun, \& S. Capkun (2014): Is bitcoin a decentralized currency? IEEE security \& privacy, vol. 12 , no. 3, pp. 54-60.

Gibbons, R. (2005): Four formal (izable) theories of the firm? Journal of Economic Behavior \& Organization, vol. 58 , no. 2 , pp. 200-245.

Gillespie, T. (2010): The politics of 'platforms'. New media \& society, vol. 12, no. 3, pp. 347-364.

Gillespie, T. (2018): Custodians of the Internet: Platforms, content moderation, and the hidden decisions that shape social media. Yale University Press. 030023502X.

Glaser, F. (2017): Pervasive decentralisation of digital infrastructures: a framework for blockchain enabled system and use case analysis.

Glaser, F. \& L. Bezzenberger Beyond cryptocurrencies-a taxonomy of decentralized consensus systems. In.

Gleeson, S. (2018): The Legal Concept of Money. Oxford University Press.

Goffman, E. (1959): The Presentation of Self in Everyday Life. New York, NY: Bantam.

Goldsmith, J. L. \& T. Wu (2006): Who Controls the Internet?: Illusions of a Borderless World. Oxford University Press, USA.

Goldsmith, J. L. \& T. Wu (2006): Who Controls the Internet?: Illusions of a Borderless World. Oxford University Press, USA.

Google Search Trends (2019): Blockchain 2004-2019, https://trends.google.com/trends/explore?date=all\&geo=US\&q=blockchain.

Gray, P. (1996): The global information infrastructure. Information Systems Management, vol. 13, no. 3, pp. 7-14.

Greenstein, S. (2009): Open platform development and the commercial Internet. Platforms, markets and innovation, pp. 219-248.

Griffin, J. M. \& A. Shams (2019): Is bitcoin really un-tethered? Available at SSRN 3195066.

Grossman, S. J. \& O. D. Hart (1986): The costs and benefits of ownership: A theory of vertical and lateral integration. Journal of political economy, vol. 94, no. 4, pp. 691-719.

Guesmi, K., S. Saadi, I. Abid, \& Z. Ftiti (2019): Portfolio diversification with virtual currency: Evidence from bitcoin. International Review of Financial Analysis, vol. 63, pp. 431-437.

Hacker, P. (2019): Corporate Governance for Complex Cryptocurrencies? A Framework for Stability and Decision Making in Blockchain-Based Organizations.

Halaburda, H. \& N. Gandal (2016): Competition in the cryptocurrency market. Available at SSRN 2506463.

Hammill, C. From Crossbows to Cryptography: Techno-Thwarting the State. In.

Hanseth, O. \& E. Monteiro (1997): Inscribing Behavior in Information Infrastructure Standards. Accounting, Management and Information Technologies, vol. 7, no. 4, pp. 183-211.

Hanseth, O. \& K. Lyytinen (2010): Design Theory for Dynamic Complexity in Information Infrastructures: The Case of Building Internet. Journal of Information Technology, vol. 25, no. 1, pp. 1-19.

Hart, H. L. A. \& L. Green (2012): The concept of law. Oxford University Press. 0199644691.

Hawlitschek, F., B. Notheisen, \& T. Teubner (2018): The limits of trust-free systems: A literature review on blockchain technology and trust in the sharing economy. Electronic commerce research and applications, vol. 29, pp. 50-63.

Hayes, A. (2019): The socio-technological lives of bitcoin. Theory, Culture \& Society, vol. 36, no. 4, pp. 49-72.

Hayes, A. S. (2017): Cryptocurrency value formation: An empirical study leading to a cost of production model for valuing bitcoin. Telematics and Informatics, vol. 34, no. 7, pp. 1308-1321.

Helmond, A. (2015): The platformization of the web: Making web data platform ready. Social Media+ Society, vol. 1, no. 2, pp. 2056305115603080. 
Helmond, A., D. B. Nieborg, \& F. N. van der Vlist (2019): Facebook's evolution: development of a platform-as-infrastructure. Internet Histories, vol. 3, no. 2, pp. 123-146.

Henfridsson, O. \& B. Bygstad (2013): The Generative Mechanisms of Digital Infrastructure Evolution. MIS Quarterly, vol. 37, no. 3, pp. 907-931.

Hohfeld, W. N. (1913): Some fundamental legal conceptions as applied in judicial reasoning. Yale Lj, vol. 23, pp. 16.

Hohfeld, W. N. (1917): Fundamental legal conceptions as applied in judicial reasoning. The Yale Law Journal, vol. 26, no. 8, pp. 710-770.

Holmstrom, B. \& P. Milgrom (1991): Multitask principal-agent analyses: Incentive contracts, asset ownership, and job design. JL Econ. \& Org., vol. 7, pp. 24.

Hsieh, Y.-Y., J.-P. J. P. Vergne, \& S. Wang (2017): The internal and external governance of blockchainbased organizations: Evidence from cryptocurrencies. In Bitcoin and Beyond (Open Access)Routledge, pp. 48-68.

Hsieh, Y.-Y., J.-P. Vergne, \& A. Shawang (2018): The Internal and External Governance of BlockchainBased Organizations: Evidence From Cryptocurrencies. In Bitcoin and beyond: Cryptocurrencies, blockchains, and global governance, ed. M. Campbell-Verduyn. Abingdon: Routledge.

Huberman, G., J. Leshno, \& C. C. Moallemi (2017): Monopoly Without a Monopolist: An Economic Analysis of the Bitcoin Payment System. SSRN Scholarly Paper ID 3025604. Social Science Research Network, Rochester, NY, URL https://papers. ssrn. com/abstract, vol. 3054887.

Jacobides, M. G. \& S. G. Winter (2005): The co-evolution of capabilities and transaction costs: Explaining the institutional structure of production. Strategic Management Journal, vol. 26, no. 5, pp. 395-413.

Jacobides, M. G., C. Cennamo, \& A. Gawer (2018): Towards a theory of ecosystems. Strategic Management Journal, vol. 39, no. 8, pp. 2255-2276.

Jensen, M. C. \& W. H. Meckling (1976): Theory of the firm: Managerial behavior, agency costs and ownership structure. Journal of financial economics, vol. 3, no. 4, pp. 305-360.

Jentzsch, C. (2016): Decentralized autonomous organization to automate governance. White paper, November.

John, T. \& M. Pam Complex adaptive blockchain governance. In. EDP Sciences, vol. 223, pp. 01010.

Kahin, B. (1998): Beyond the National Information Infrastructure. In Investing in Innovation. Creating Research and Innovation Policy that Works, ed. L. M. Branscomb and K. H. Keller. Cambridge, MA: MIT Press, pp. 339-360.

Kavanagh, D., G. Miscione, \& P. J. Ennis (2019): The Bitcoin game: Ethno-resonance as method. Organization. https://doi-org.gate3.library.Ise.ac.uk/10.1177/1350508419828567

Kazan, E., C.-W. Tan, E. T. K. Lim, C. Sørensen, \& J. Damsgaard (2018): Disentangling Digital Platform Competition: The Case of UK Mobile Payment Platforms. Journal of Management Information Systems - special issue on Financial IS, Underlying Technologies, and the FinTech Revolution, vol. 35, no. 1, pp. 180-219.

Kewell, B., R. Adams, \& G. Parry (2017): Blockchain for good? Strategic Change, vol. 26, no. 5, pp. 429-437.

Klein, T., H. P. Thu, \& T. Walther (2018): Bitcoin is not the New Gold-A comparison of volatility, correlation, and portfolio performance. International Review of Financial Analysis, vol. 59, pp. 105-116.

Kroll, J. A., I. C. Davey, \& E. W. Felten (2013). The economics of Bitcoin mining, or Bitcoin in the presence of adversaries. In, vol. 2013, pp. 11.

Kubát, M. (2015): Virtual currency bitcoin in the scope of money definition and store of value. Procedia Economics and Finance, vol. 30, pp. 409-416.

Kwon, Y., J. Liu, M. Kim, D. Song, \& Y. Kim (2019): Impossibility of Full Decentralization in Permissionless Blockchains. arXiv preprint arXiv:1905.05158.

Lacity, M. C. (2018): A Manager's Guide to Blockchains for Business: From Knowing What to Knowing How. Stratford-upon-Avon, UK: SB Publishing. 1540-1960. 
Lacity, M. C. (2018): Addressing Key Challenges to Making Enterprise Blockchain Applications a Reality. MIS Quarterly Executive, vol. 17, no. 3.

Lacity, M., R. Sabherwal, \& C. Sørensen (2019): Special Issue Editorial: Delivering Business Value through Enterprise Blockchain Applications. MIS Quarterly Executive, vol. 18, no. 4.

Langlois, R. N. (2003): The vanishing hand: the changing dynamics of industrial capitalism. Industrial and corporate change, vol. 12, no. 2, pp. 351-385.

Lessig, L. (2006): Code: version 2.0. New York: Basic Books.

$\mathrm{Li}, \mathrm{X}$. \& C. A. Wang (2017): The technology and economic determinants of cryptocurrency exchange rates: The case of Bitcoin. Decision Support Systems, vol. 95, pp. 49-60.

Linder, T. (2019): Surveillance Capitalism and Platform Policing: The Surveillant Assemblage-as-aService. Surveillance \& Society, vol. 17, no. 1/2, pp. 76-82.

Lo, S. \& J. C. Wang (2014): Bitcoin as money?

Locke, J. (1947): Two Treatises of Government: With a Supplement, Patriarcha, by Robert Filmer. Simon and Schuster. 0028485009. 2.

Luther, W. J. (2016): Cryptocurrencies, network effects, and switching costs. Contemporary Economic Policy, vol. 34, no. 3, pp. 553-571.

Lyytinen, K., C. Sørensen, \& D. Tilson (2018): The Generativity of Digital Infrastructures: A Research Note. In Handbook on IS Companion Book. Chapter 17, ed. R. D. Galliers and M.-K. SteinRoutledge. https://www.routledge.com/The-Routledge-Companion-to-ManagementInformation-Systems/Galliers-Stein/p/book/9781138666450

Ma, J., J. S. Gans, \& R. Tourky (2018): Market structure in bitcoin mining. National Bureau of Economic Research.

Maskin, E. \& J. Tirole (1999): Unforeseen contingencies and incomplete contracts. The Review of Economic Studies, vol. 66, no. 1, pp. 83-114.

Mathew, A. J. (2016): The myth of the decentralised internet. Internet Policy Review, vol. 5, no. 3.

Mathiassen, L. \& C. Sørensen (2008): Towards A Theory of Organizational Information Services. Journal of Information Technology, vol. 23, no. 4, pp. 313-329.

Maurer, B., T. C. Nelms, \& L. Swartz (2013): “When perhaps the real problem is money itself!": the practical materiality of Bitcoin. Social semiotics, vol. 23, no. 2, pp. 261-277.

May, T. (1988a): Libertaria in Cyberspace or Cyberspace more hospitable to ideas of liberty and crypto anarchy, Satoshi Nakamoto Institute, https://nakamotoinstitute.org/libertaria-incyberspace/

May, T. (1988b): The Crypto Anarchist Manifesto, Satoshi Nakamoto Institute: https://nakamotoinstitute.org/crypto-anarchist-manifesto/

May, T. C. (1994), Crypto anarchy and virtual communities. Available at: http://aom.jku.at/archiv/cmc/text/may n01.pdf

McWaters, R. J., G. Bruno, R. Galaski, \& S. Chaterjee The future of financial infrastructure: An ambitious look at how blockchain can reshape financial services. In, vol. 49.

Meunier, S. \& D. Zhao-Meunier (2019): Bitcoin, Distributed Ledgers and the Theory of the Firm. Available at SSRN 3327971.

Mill, J. S. (1867): On Liberty. Longmans, Green \& Company.

Mintzberg, H. (1983): Structure in fives: designing effective organizations. Englewood Cliffs, New Jersey: Prentice-Hall.

Miscione, G., R. Ziolkowski, L. Zavolokina, \& G. Schwabe (2018): Tribal governance: The business of blockchain authentication. In Proceedings of the 51st Hawaii International Conference on System Sciences. http://hdl.handle.net/10125/50455

Miscione, G., T. Goerke, S. Klein, G. Schwabe, \& R. Ziolkowski (2019): Hanseatic Governance: Understanding Blockchain as Organizational Technology.

Möller, J. \& M. Rimscha (2017): (De) centralization of the global informational ecosystem. Media and Communication, vol. 5, no. 3, pp. 37-48. 
Monegro, J., F. Wilson, A. Wenger, \& M. Ali (2014): The blockchain application stack. CoinDesk, November, vol. 20.

Musiani, F., A. Mallard, \& C. Méadel (2018): Governing what wasn't meant to be governed: A controversy-based approach to the study of Bitcoin governance. In Bitcoin and beyond: Cryptocurrencies, blockchains, and global governance, ed. M. Campbell-Verduyn. Abingdon: Routledge. pp. 133-156.

Musiani, F., D. L. Cogburn, L. DeNardis, \& N. S. Levinson (2016): The turn to infrastructure in Internet governance. Springer.

Nabilou, H. (2019): How to Regulate Bitcoin? Decentralized Regulation for a Decentralized Cryptocurrency. Decentralized Regulation for a Decentralized Cryptocurrency (March 26, 2019).

Nabilou, H. \& A. Prüm (2019): Ignorance, Debt, and Cryptocurrencies: The Old and the New in the Law and Economics of Concurrent Currencies. Journal of Financial Regulation, vol. 5, no. 1, pp. 29-63.

Nakamoto, S. (2008): Bitcoin: A Peer-to-Peer Electronic Cash System. https://bitcoin.org/bitcoin.pdf

Narayanan, A., J. Bonneau, E. Felten, A. Miller, \& S. Goldfeder (2016): Bitcoin and cryptocurrency technologies: a comprehensive introduction. Princeton University Press. 1400884152.

NASDAQ (Feb. 26, 2016), https://www.nasdaq.com/article/why-some-changes-to-bitcoinrequire-

Nechushtai, E. (2018): Could digital platforms capture the media through infrastructure? Journalism, vol. 19, no. 8, pp. 1043-1058.

Neudecker, T. \& H. Hartenstein (2018): Network layer aspects of permissionless blockchains. IEEE Communications Surveys \& Tutorials, vol. 21 , no. 1, pp. 838-857.

Nieborg, D. B. \& A. Helmond (2019): The political economy of Facebook's platformization in the mobile ecosystem: Facebook Messenger as a platform instance. Media, Culture \& Society, vol. 41, no. 2, pp. 196-218.

Nieborg, D. B. \& T. Poell (2018): The platformization of cultural production: Theorizing the contingent cultural commodity. New Media \& Society, vol. 20, no. 11, pp. 4275-4292.

Notheisen, B., F. Hawlitschek, \& C. Weinhardt (2017): Breaking down the blockchain hype-towards a blockchain market engineering approach.

Ocho, R. (2017): Architectural Evolution through Softwarisation: On the Advent of Software-Defined Networks. PhD Thesis, London School of Economics and Political Science. http://etheses.Ise.ac.uk/3594/

Okada, H., S. Yamasaki, \& V. Bracamonte Proposed classification of blockchains based on authority and incentive dimensions. In. IEEE, pp. 593-597.

Okada, H., S. Yamasaki, \& V. Bracamonte Proposed classification of blockchains based on authority and incentive dimensions. In. IEEE, pp. 593-597.

$\varnothing$ stbye, P. (2019): Who is Liable if a Cryptocurrency Protocol Fails? Available at SSRN 3423681.

Pagnotta, E. S. (2018): Bitcoin as decentralized money: prices, mining, and network security. SSRN (3264448).

Parker, G. G., M. W. Van Alstyne, \& S. P. Choudary (2016): Platform revolution: how networked markets are transforming the economyand how to make them work for you. WW Norton \& Company. 0393249123.

Pedersen, A., M. Risius, \& R. Beck (2019): Blockchain Decision Path: When to Use Blockchains? Which blockchains do you mean? MIS Quarterly Executive, vol. 18, no. 2.

Pereira, J., M. M. Tavalaei, \& H. Ozalp (2019): Blockchain-based platforms: Decentralized infrastructures and its boundary conditions. Technological Forecasting and Social Change, vol. 146, pp. 94-102.

Phillip, A., J. S. K. Chan, \& S. Peiris (2018): A new look at Cryptocurrencies. Economics Letters, vol. 163, pp. 6-9.

Plantin, J.-C. \& A. Punathambekar (2019): Digital media infrastructures: pipes, platforms, and politics. Media, Culture \& Society, vol. 41, no. 2, pp. 163-174. 
Plantin, J.-C. \& G. de Seta (2019): WeChat as infrastructure: the techno-nationalist shaping of Chinese digital platforms. Chinese Journal of Communication, pp. 1-17.

Plantin, J.-C., C. Lagoze, P. N. Edwards, \& C. Sandvig (2018): Infrastructure studies meet platform studies in the age of Google and Facebook. New Media \& Society, vol. 20, no. 1, pp. 293-310.

Polasik, M., A. I. Piotrowska, T. P. Wisniewski, R. Kotkowski, \& G. Lightfoot (2015): Price fluctuations and the use of Bitcoin: An empirical inquiry. International Journal of Electronic Commerce, vol. 20, no. 1, pp. 9-49.

Qu, Q., I. Nurgaliev, M. Muzammal, C. S. Jensen, \& J. Fan (2019): On spatio-temporal blockchain query processing. Future Generation Computer Systems, vol. 98, pp. 208-218.

Randhawa, K., R. Wilden, \& J. Hohberger (2016): A bibliometric review of open innovation: Setting a research agenda. Journal of Product Innovation Management, vol. 33, no. 6, pp. 750-772.

Raskin, M. \& D. Yermack (2018): Digital currencies, decentralized ledgers and the future of central banking. In Research Handbook on Central BankingEdward Elgar Publishing.

Rauchs, M., A. Glidden, B. Gordon, G. C. Pieters, M. Recanatini, F. Rostand, K. Vagneur, \& B. Z. Zhang (2018): Distributed ledger technology systems: a conceptual framework.

Reijers, W., F. O'Brolcháin, \& P. Haynes (2016): Governance in blockchain technologies \& social contract theories. Ledger, vol. 1, pp. 134-151.

Reijers, W., I. Wuisman, M. Mannan, P. De Filippi, C. Wray, V. Rae-Looi, A. C. VÈlez, \& L. Orgad (2018): Now the Code Runs Itself: On-Chain and Off-Chain Governance of Blockchain Technologies. Topoi, pp. 1-11.

Reijers, W., I. Wuisman, M. Mannan, P. De Filippi, C. Wray, V. Rae-Looi, A. C. Vélez, \& L. Orgad (2018): Now the code runs itself: On-chain and off-chain governance of blockchain technologies. Topoi, pp. 1-11.

Reyes, C. (2019): (Un) Corporate Crypto-Governance. Fordham Law Review, Forthcoming.

Reyes, C. L. (2016): Moving beyond bitcoin to an endogenous theory of decentralized ledger technology regulation: an initial proposal. Vill. L. Rev., vol. 61, pp. 191.

Reyes, C. L. (2019): If Rockefeller Were a Coder. Geo. Wash. L. Rev., vol. 87, pp. 373.

Reyes, C. L., N. Geslevich Packin, \& B. P. Edwards (2017): Distributed Governance. Wm. \& Mary L. Rev. Online, vol. 59, pp. 1.

Risius, M. \& K. Spohrer (2017): A blockchain research framework. Business \& Information Systems Engineering, vol. 59, no. 6, pp. 385-409.

Rochet, J.-C. \& J. Tirole (2003): Platform Competition in Two-Sided Markets. Journal of the European Economic Association, vol. 1, no. 4, pp. 990-1029.

Rochet, J.-C. \& J. Tirole (2006): Two-Sided Markets: A Progress Report. The RAND Journal of Economics, vol. 37, no. 3, pp. 645-667.

Roda, F. (2019): Should Crypto Assets Continue To Be Treated As Commodities; The Role Of Consensus Algorithms In The Classification Of Assets, At:

https://www.financederivative.com/should-crypto-assets-continue-to-be-treated-ascommodities-the-role-of-consensus-algorithms-in-the-classification-of-assets/

Rodrigues, U. R. (2018): Law and the Blockchain. lowa L. Rev., vol. 104, pp. 679.

Schilling, L. \& H. Uhlig (2019): Some simple bitcoin economics. Journal of Monetary Economics, vol. 106, pp. 16-26.

Schneider, A. (2003): Decentralization: Conceptualization and measurement. Studies in comparative international development, vol. 38, no. 3, pp. 32-56.

Schneider, N. (2019): Decentralization: an incomplete ambition. Journal of Cultural Economy, pp. 121.

Searle, J. R. (1969): How to derive 'ought'from 'is'. In The is-ought questionSpringer, pp. 120-134.

Shapiro, C. \& H. R. Varian (1998): Information Rules: A Strategic Guide to the Network Economy. Boston: Harvard Business School Press.

Simmel, G. (2004): The philosophy of money. Routledge. 0203481135. 
Smith, A. E. \& M. S. Humphreys (2006): Evaluation of unsupervised semantic mapping of natural language with Leximancer concept mapping. Behavior Research Methods, vol. 38, no. 2, pp. 262-279.

Srinivasan, B. \& L. Lee (2017): Quantifying Decentralization. Medium. https://news.earn.com/quantifying-decentralization-e39db233c28e

Srinivasan, B. \& L. Lee (2017): Quantifying Decentralization.(2017).

Srnicek, N. (2017): Platform capitalism. John Wiley \& Sons. 1509504885.

Stewart, I. (2007): Why Beauty Is Truth: The History of Symmetry. Basic Books Inc.

Sundararajan, A. (2013): From Zipcar to the sharing economy. Harvard Business Review, vol. 1.

Sutton, J. (1991): Sunk costs and market structure: Price competition, advertising, and the evolution of concentration. MIT press. 0262193051.

Sutton, J. (1997): Gibrat's legacy. Journal of economic literature, vol. 35, no. 1, pp. 40-59.

Swan, M. (2015): Blockchain: Blueprint for a new economy. " O'Reilly Media, Inc.". 1491920475.

Swan, M. \& P. De Filippi (2017): Towards a Philosophy of Blockchain.

Swartz, L. (2018): What was Bitcoin, what will it be? The techno-economic imaginaries of a new money technology. Cultural Studies, vol. 32, no. 4, pp. 623-650.

Szabo, N. (1996): Smart contracts: building blocks for digital markets. EXTROPY: The Journal of Transhumanist Thought. (16).

Szabo, N. (1997): The god protocols. Consultado en: https://nakamotoinstitute. org/the-godprotocols.

Szabo, N. (1998): Secure Property Titles with Owner Authority.(1998). Источник https://nakamotoinstitute. org/secure-property-titles.

Szabo, N. (2001): Trusted third parties are security holes. URL http://nakamotoinstitute. org/trustedthird-parties.

Szabo, N. (2002): Shelling out: the origins of money. Satoshi Nakamoto Institute.

Szabo, N. (2003): Advances in distributed security. Retrieved from.

Szabo, N. (2005): Bit Gold.(2005).

Tanenbaum, A. S. (2011): Computer Networks, /Andrew S. Tanenbaum, David J. Wetherall. Cloth: Prentice Hall.

Tanenbaum, A. S. \& M. Van Steen (2007): Distributed systems: principles and paradigms. PrenticeHall. 0132392275.

Tapscott, D. \& A. Tapscott (2017): How blockchain will change organizations. MIT Sloan Management Review, vol. 58, no. 2, pp. 10.

Tasca, P. \& C. J. Tessone (2017): Taxonomy of blockchain technologies. Principles of identification and classification. https://arxiv.org/pdf/1708.04872.pdf

Tasca, P., T. Thanabalasingham, \& C. J. Tessone (2017): Ontology of Blockchain Technologies. Principles of identification and classification. SSRN Electronic Journal, vol. 10.

Thomas, D. A. (2014): Searching for significance in unstructured data: text mining with leximancer. European Educational Research Journal, vol. 13, no. 2, pp. 235-256.

Thomas, L., E. Autio, \& D. Gann (2014): Architectural Leverage: Putting Platforms in Context. The Academy of Management Perspectives, vol. 28, no. 2, pp. 198-219.

Tilson, D., K. Lyytinen, \& C. Sørensen (2010): Digital Infrastructures: The Missing IS Research Agenda. Information Systems Research, vol. 21, no. 5, pp. 748-759.

Tirole, J. (1999): Incomplete contracts: Where do we stand? Econometrica, vol. 67, no. 4, pp. 741781.

Tiwana, A., B. Konsynsky, \& A. A. Bush (2010): Platform Evolution: Coevolution of Platform Architecture, Governance, and Environmental Dynamics. Information Systems Research, vol. 21, no. 4, pp. 675-687.

Torrisi, G., A. Pike, J. Tomaney, \& V. Tselios (2011): Defining and measuring decentralisation: a critical review. 
Troncoso, C., M. Isaakidis, G. Danezis, \& H. Halpin (2017): Systematizing decentralization and privacy: Lessons from 15 years of research and deployments. Proceedings on Privacy Enhancing Technologies, vol. 2017, no. 4, pp. 404-426.

Trottier, D. (2016): Social media as surveillance: Rethinking visibility in a converging world. Routledge. Trottier, D. (2019): A research agenda for social media surveillance. Fast Capitalism, vol. 8, no. 1.

Tschorsch, F. \& B. Scheuermann (2016): Bitcoin and beyond: A technical survey on decentralized digital currencies. IEEE Communications Surveys \& Tutorials, vol. 18, no. 3, pp. 2084-2123.

Van Dijck, J. (2013): The culture of connectivity: A critical history of social media. Oxford University Press. 0199970793.

Van Dijck, J., T. Poell, \& M. De Waal (2018): The platform society: Public values in a connective world. Oxford University Press. 0190889780.

Van Schewick, B. (2012): Internet architecture and innovation. Mit Press. 0262265575.

Van Valkenburgh, P. (2016): Framework for Securities Regulation of Cryptocurrencies. Coin Cen.

van Wirdum, A. Why Some Changes to Bitcoin Require Consensus: Bitcoin's 4 Layers,

Vidan, G. \& V. Lehdonvirta (2019): Mine the gap: Bitcoin and the maintenance of trustlessness. New Media \& Society, vol. 21, no. 1, pp. 42-59.

Walch, A. (2015): The bitcoin blockchain as financial market infrastructure: A consideration of operational risk. NYUJ Legis. \& Pub. Pol'y, vol. 18, pp. 837.

Walch, A. (2016): The path of the blockchain lexicon (and the law). Rev. Banking \& Fin. L., vol. 36, pp. 713.

Walch, A. (2017): Blockchain's Treacherous Vocabulary: One More Challenge for Regulators.

Walch, A. (2018): In Code (Rs) We Trust: Software Developers as Fiduciaries in Public Blockchains.

Walch, A. (2019): Deconstructing 'Decentralization': Exploring the Core Claim of Crypto Systems. Crypto Assets: Legal and Monetary Perspectives (OUP, forthcoming 2019).

Walch, A. (2019): Deconstructing'Decentralization': Exploring the Core Claim of Crypto Systems. Crypto Assets: Legal and Monetary Perspectives (OUP, forthcoming 2019).

Walch, A. (2019): In Code (Rs) We Trust: Software Developers as Fiduciaries in Public Blockchains.

Waldron, J. (1985): Theories of rights.

Walsh, C., P. Oreilly, R. Gleasure, J. Feller, S. Li, \& J. Cristoforo (2016): New kid on the block: a strategic archetypes approach to understanding the Blockchain.

Walsh, C., P. Oreilly, R. Gleasure, J. Feller, S. Li, \& J. Cristoforo (2016): New kid on the block: a strategic archetypes approach to understanding the Blockchain.

Weber, B. (2014): Bitcoin and the legitimacy crisis of money. Cambridge Journal of Economics, vol. 40, no. 1 , pp. 17-41.

Wei, W. C. (2018): Liquidity and market efficiency in cryptocurrencies. Economics Letters, vol. 168, pp. 21-24.

Wei, W. C. (2018): The impact of Tether grants on Bitcoin. Economics Letters, vol. 171, pp. 19-22.

Werbach, K. (2018): Trust, but verify: Why the blockchain needs the law. Berkeley Tech. LJ, vol. 33, pp. 487.

Werbach, K. \& N. Cornell (2017): Contracts ex machina. Duke LJ, vol. 67, pp. 313.

West, J. \& M. Bogers (2014): Leveraging external sources of innovation: a review of research on open innovation. Journal of Product Innovation Management, vol. 31, no. 4, pp. 814-831.

Weyl, H. (1983): Symmetry. Princeton University Press.

White, L. H. (2015): The market for cryptocurrencies. Cato J., vol. 35, pp. 383.

Williamson, O. (1985): E.(1985): The Economic Institutions of Capitalism. New York.

Williamson, O. E. (1979): Transaction-cost economics: the governance of contractual relations. The journal of Law and Economics, vol. 22, no. 2, pp. 233-261.

Wood, D. M. \& T. Monahan (2019): Platform Surveillance. Surveillance \& Society, vol. 17, no. 1/2, pp. 1-6.

Wood, G. (2014): Ethereum: A secure decentralised generalised transaction ledger. Ethereum project yellow paper, vol. 151, no. 2014, pp. 1-32. 
Wright, A. \& P. De Filippi (2015): Decentralized blockchain technology and the rise of lex cryptographia. Available at SSRN 2580664.

Wright, A. \& P. De Filippi (2015): Decentralized blockchain technology and the rise of lex cryptographia. Available at SSRN 2580664.

Wu, T. (2003): When code isn't law. Va. L. Rev., vol. 89, pp. 679.

Wu, T. (2010): The Master Switch: The Rise and Fall of Information Empires. Knopf Publishing Group.

Wuehrer, G. A. \& A. E. Smejkal (2013): The knowledge domain of the academy of international business studies (AIB) conferences: a longitudinal scientometric perspective for the years 2006ñ2011. Scientometrics, vol. 95, no. 2, pp. 541-561.

$\mathrm{Xu}, \mathrm{X}$., I. Weber, M. Staples, L. Zhu, J. Bosch, L. Bass, C. Pautasso, \& P. Rimba A taxonomy of blockchain-based systems for architecture design. In. IEEE, pp. 243-252.

Yermack, D. (2017): Corporate governance and blockchains. Review of Finance, vol. 21, no. 1, pp. 731.

Yeung, K. (2019): Regulation by Blockchain: the Emerging Battle for Supremacy between the Code of Law and Code as Law. The Modern Law Review, vol. 82, no. 2, pp. 207-239.

Yi, S., Z. Xu, \& G.-J. Wang (2018): Volatility connectedness in the cryptocurrency market: Is Bitcoin a dominant cryptocurrency? International Review of Financial Analysis, vol. 60, pp. 98-114.

Zachariadis, M., G. Hileman, \& S. V. Scott (2019): Governance and control in distributed ledgers: understanding the challenges facing blockchain technology in financial services. Information and Organization, vol. 29, no. 2, pp. 105-117.

Zetzsche, D. A., R. P. Buckley, \& D. W. Arner (2018): The distributed liability of distributed ledgers: Legal risks of blockchain. U. III. L. Rev., pp. 1361.

Zetzsche, D. A., R. P. Buckley, D. W. Arner, \& L. Föhr (2017): The ICO Gold Rush: It's a scam, it's a bubble, it's a super challenge for regulators. University of Luxembourg Law Working Paper, no. 11, pp. 17-83.

Ziolkowski, R., G. Miscione, \& G. Schwabe (2018): Consensus through Blockchains: Exploring Governance across inter-organizational Settings.

Ziolkowski, R., G. Parangi, G. Miscione, \& G. Schwabe (2019): Examining Gentle Rivalry: DecisionMaking in Blockchain Systems. In 52nd Hawaii International Conference on System Sciences (HICSS 2019), Maui, Hawaii, 8 January 2019 - 13 January, pp. 1-10. https://doi.org/10.5167/uzh160377

Ziolkowski, R., G. Parangi, G. Miscione, \& G. Schwabe Examining Gentle Rivalry: Decision-Making in Blockchain Systems. In, pp. 1-10.

Zittrain, J. (2006): The Generative Internet. Harvard Law Review, vol. 119, pp. 1974-2040.

Zittrain, J. (2008): The Future of the Internet: And How to Stop It. London: Allen Lane.

Zittrain, J. (2018): Fixing the internet. American Association for the Advancement of Science,

Zittrain, J. \& J. Palfrey (2008): Internet filtering: The politics and mechanisms of control. Access denied: The practice and policy of global Internet filtering, vol. 41.

Zittrain, J. L. (2019): A History of IP. In A History of Intellectual Property in 50 Objects. Chapter 45. https://ssrn.com/abstract=3373352

Zuboff, S. (2019): The age of surveillance capitalism: The fight for a human future at the new frontier of power. London: Profile Books. 